\title{
Um espaço de Banach não isomorfo ao conjugado complexo
}

\author{
Wilson Albeiro Cuellar Carrera
}

DisSERTAÇÃO APRESENTADA

$\mathrm{AO}$

Instituto DE MATEMÁtica e Estatística

DA

Universidade DE SÃo PaUlo

PARA

OBTENÇÃO DO TÍTULO

$\mathrm{DE}$

Mestre em CiÊnCIAS

Programa: Matemática

Orientador: Prof. Dr. Valentin Raphael Henri Ferenczi

Durante o desenvolvimento deste trabalho o autor recebeu auxílio financeiro do CNPq

São Paulo, Fevereiro de 2011 


\section{Um espaço de Banach não isomorfo ao conjugado complexo}

Esta versão definitiva da dissertação contém as correções e alterações sugeridas pela Comissão Julgadora durante a defesa realizada por Wilson Albeiro Cuellar Carrera em 25/02/2011.

Comissão Julgadora:

- Profa. Dr. Valentin Raphael Henri Ferenczi (orientador) - IME-USP

- Prof. Dr. Eloi Medina Galego - IME-USP

- Prof. Dr. Jorge Tulio Mujica Ascui - UNICAMP 


\section{Agradecimentos}

Agradeço inicialmente ao meu orientador, o Prof. Dr. Valentin Ferenczi, pela sua dedicação e disposição para todas as minhas consultas.

A minha família pelo incentivo e apoio em todo momento.

Aos amigos e colegas do instituto pela atitude amável e integradora.

Agradeço o apoio financiero do CNPq através da bolsa de mestrado. 


\section{Resumo}

Neste trabalho fazemos um estudo do conceito de soma torcida de $F$-espaços. Apresentamos algumas propriedades e simplificações na construção de somas torcidas de $F$-espaços localmente limitados. Em particular, estudamos uma condição suficiente para que uma soma torcida de espaços de Banach seja um espaço de Banach. Finalmente aplicamos esses conceitos para definir o espaço construído por N. J. Kalton [Ka195], que é um exemplo de um espaço de Banach não isomorfo ao conjugado complexo. Este espaço $X$ de Kalton corresponde a uma soma torcida de espaços de Hilbert, isto é, $X$ possui um subespaço fechado $E$ tal que $E$ e $X / E$ são isomorfos a espaços de Hilbert.

Palavras-chave: Estruturas complexas, Somas torcidas, Espaços de Banach. 


\section{Abstract}

In this work we study the concept of twisted sum of $F$-spaces. We present some properties and simplifications in the construction of twisted sums of locally bounded $F$-spaces. In particular, we study a sufficient condition for a twisted sum of Banach spaces to be a Banach space. Finally we apply these concepts to define the space constructed by N. J. Kalton [Ka195], which is an example of a Banach space not isomorphic to its complex conjugate. The Kalton space $X$ is a twisted sum of Hilbert spaces, i.e. $X$ has a closed subspace $E$ such that $E$ and $X / E$ are isomorphic to Hilbert spaces.

Keywords: Complex structure, Twisted sums, Banach spaces. 


\section{Sumário}

1 Resultados preliminares $\quad 3$

1.1 Conceitos e notações básicas . . . . . . . . . . . . . . . . . . 3

1.2 Espaços vetoriais topológicos . . . . . . . . . . . . . . . . . . . 7

1.3 Espaços Quase-normados . . . . . . . . . . . . . . . . . . . . . . . . . . 12

1.4 Decomposição de Schauder . . . . . . . . . . . . . . . . . . . . . . . 17

2 Somas torcidas $\quad 25$

2.1 Somas torcidas . . . . . . . . . . . . . . . . . . . 25

2.2 Propriedades preservadas pelas somas torcidas . . . . . . . . . . . . 30

2.3 Somas torcidas de espaços de Banach . . . . . . . . . . . . . . . . 32

2.4 Simplificações na construção de somas torcidas . . . . . . . . . . . . . . . . . 44

3 Exemplo de um espaço de Banach não isomorfo a seu conjugado complexo 57

3.1 Construção do espaço $Z_{2}(\alpha) \ldots \ldots \ldots \ldots \ldots$. . . . . . . . . . . . . . . . . . . . . . . .

3.2 Propriedades do espaço $Z_{2}(\alpha) \ldots \ldots \ldots \ldots \ldots$

3.3 Isomorfismos entre os espaços $Z_{2}(\alpha) \ldots \ldots \ldots$. . . . . . . . . . 60

$\begin{array}{ll}\text { Referências Bibliográficas } & 73\end{array}$ 


\section{Introdução}

Um resultado clássico de S. Mazur e S. Ulam [MU32] estabelece que dois espaços de Banach reais que sejam isométricos devem ser $\mathbb{R}$-linearmente isométricos. A pergunta natural é se esse resultado pode-se estender ao caso complexo. Dado um espaço de Banach complexo $X$, o espaço mais simples que é $\mathbb{R}$-linearmente isométrico a $X$ é o espaço conjugado $\bar{X}$ de $X$, que está definido como o espaço $X$ munido com a lei escalar $\lambda \odot x=\bar{\lambda} x$ em vez de $\lambda x$. Em 1986 J. Bourgain [Bou86] exibiu dois espaços de Banach que são linearmente isométricos como espaços reais, porém não isomorfos como espaços complexos. Mais especificamente, Bourgain construiu, usando técnicas probabilísticas, um espaço de Banach não isomorfo ao conjugado. Desta forma, o resultado de Mazur-Ulam não é válido no caso complexo. Uma construção mais explícita de um espaço não isomorfo ao conjugado foi obtida por N. J. Kalton [Kal95] mediante somas torcidas. O objetivo deste trabalho é apresentar o espaço de Kalton, que é uma soma torcida de $\ell_{2} \operatorname{com} \ell_{2}$.

Este trabalho está dividido em três capítulos. No capítulo 1, estabelecemos a notação e os conceitos fundamentais, começando com as propriedades gerais dos espaços vetoriais topológicos(e.v.t), em particular mostramos uma condição necessária e suficiente para que um e.v.t seja normalizável. Vamos centrar a nossa atenção nos espaços localmente limitados e sua correspondência com os espaços quase-normados. Demonstramos o teorema de AokiRolewicz que estabelece que todo espaço localmente limitado é localmente $p$-convexo para algum $p(0<p \leq 1)$. Também estudamos a noção de espaço de Banach com decomposição de Schauder. Estabelecemos um teorema de 'blocking' para operadores contínuos entre espaços com decomposição contrátil.

No capítulo 2, fazemos um estudo das somas torcidas em $F$-espaços. Listamos algumas propriedades que são preservadas pelas somas torcidas, tais como ser reflexivo, localmente limitado, completo, separável entre outras. Mostramos que toda suma torcida é gerada por um operador quase-linear. Escrevemos para o caso complexo a simplificação na construção de aplicações quase-lineares dada por Kalton e Peck [KP79]. Em particular, usando este procedimento obtemos um método para construir somas torcidas em espaços de sequências. Por um exemplo de Ribe [Rib79] existe uma soma torcida de espaços de Banach que não é um espaço de Banach. Para resolver isso é introduzido o conceito de B-convexidade e é provado um teorema de Kalton [Kal78] que estabelece que toda soma torcida de espaços de 
Banach B-convexos é um espaço de Banach B-convexo.

No capítulo 3, utilizando as técnicas obtidas na construção de somas torcidas em espaços de sequências, apresentamos os espaços de Kalton [Kal95] $Z_{2}(\alpha)(\alpha \in(-\infty, \infty))$ que são isomorfos a somas torcidas de $\ell_{2}$ com $\ell_{2}$. Provamos que o conjugado de $Z_{2}(\alpha)$ é isomorfo a $Z_{2}(-\alpha)$ e, por último estabelecemos que $Z_{2}(\alpha)$ é isomorfo a $Z_{2}\left(\alpha^{\prime}\right)$ somente se $\alpha=\alpha^{\prime}$. Com isto $Z_{2}(\alpha)$ não é isomorfo ao conjugado complexo quando $\alpha \neq 0$. 


\section{Capítulo 1}

\section{Resultados preliminares}

Neste capítulo apresentamos definições e resultados básicos que utilizaremos no decorrer deste trabalho. Em particular, introduzimos o conceito de quase-norma e observamos que um espaço vetorial topológico é localmente limitado se, e somente se, a topologia é gerada por uma quase-norma. Demonstramos o resultado clássico de Aoki-Rolewicz que induz uma métrica invariante em cada espaço localmente limitado. Quando essa métrica é completa, obtemos exemplos de $F$-espaços localmente limitados e será nesse tipo de espaços que definiremos somas torcidas no capítulo 2.

Na última seção abordaremos alguns resultados envolvendo espaços com decomposição de Schauder contrátil. Esses resultados serão fundamentais no estudo do espaço de Kalton que será definido no capítulo 3.

\subsection{Conceitos e notações básicas}

Denotaremos por $\mathbb{N}$ o conjunto dos números naturais, por $\mathbb{R}$ o conjunto dos números reais e por $\mathbb{C}$ o conjunto dos números complexos. Em geral escreveremos $\mathbb{K}$ para denotar $\mathbb{R}$ ou $\mathbb{C}$ de acordo ao contexto. Dado um número real $\alpha$, a parte inteira de $\alpha$ será escrita como $[\alpha]$.

Denotaremos por $c_{00}(\mathbb{K})$ o conjunto das sequências sobre o corpo $\mathbb{K}$, tais que suas coordenadas são todas zero, exceto um número finito.

Seja $X$ um espaço vetorial sobre um corpo $\mathbb{K}$, denotaremos por $i d_{X}$ a função identidade de $X$, isto é, a aplicação $i d_{X}: X \rightarrow X$ definida por $i d_{X}(x)=x$ para todo $x \in X$.

Definição 1.1. Sejam $X_{1}$ e $X_{2}$ subespaços de um espaço vetorial $X$ sobre um corpo $\mathbb{K}$. Dizemos que $X$ é soma direta algébrica de $X_{1}$ e $X_{2}$, e escrevemos $X=X_{1} \oplus X_{2}$, se $X_{1} \cap X_{2}=$ $\emptyset$ e cada $x \in X$ pode ser escrito da forma $x=x_{1}+x_{2}$ onde $x_{1} \in X_{1}$ e $x_{2} \in X_{2}$. 
Teorema 1.2 (Lema de Zorn). Seja P um conjunto não vazio parcialmente ordenado com a propriedade que todo subconjunto totalmente ordenado de $P$ possui uma cota superior em P. Então P contém pelo menos um elemento maximal.

Segue do Lema de Zorn que todo espaço vetorial possui uma base de Hamel, ou seja, uma família geradora e linearmente independente. De modo mais geral, temos o seguinte:

Teorema 1.3. Sejam $X$ um espaço vetorial sobre um corpo $\mathbb{K}$ e $E$ um subconjunto de $X$ linearmente independente. Então existe uma base de Hamel $B$ de $X$ tal que $E \subseteq B$.

Os seguintes três lemas são consequências do teorema anterior.

Lema 1.4. Sejam $X$ um espaço vetorial e $X_{1}$ um subespaço de $X$. Então existe um subespaço $X_{2}$ de $X$, tal que $X=X_{1} \oplus X_{2}$.

Lema 1.5. Sejam $X$ e $Y$ espaços vetoriais e $\widetilde{T}: X_{0} \rightarrow Y$ uma aplicação linear definida em $X_{0}$ um subespaço vetorial de $X$. Então existe uma extensão linear $T: X \rightarrow Y$, isto é, $T$ é uma aplicação linear tal que $T(x)=\widetilde{T}(x)$ para todo $x \in X_{0}$.

Lema 1.6. Seja $T: X \rightarrow Y$ uma aplicação linear sobrejetora entre espaços vetoriais $X$ e $Y$. Então existe uma aplicação linear $S: Y \rightarrow X$ tal que $T \circ S=i d_{Y}$.

Sejam $X$ um espaço vetorial sobre um corpo $\mathbb{K}$ e $A$ e $B$ subconjuntos de $X$. Indicaremos por span $A$ o subespaço gerado por $A$ em $X$. $A+B$ será o conjunto de todas as somas de elementos de $A$ e $B$, ou seja, $A+B=\{x+y ; \quad x \in A, y \in B\}$. No caso $B=\{x\}$, escreveremos simplesmente $A+B=A+x$. Para cada $\lambda \in \mathbb{K}$ definimos $\lambda A=\{\lambda x ; \quad x \in A\}$.

Definição 1.7. A envoltória convexa de um subconjunto $C$ de um espaço vetorial $X$ é o conjunto

$$
\left\{\alpha_{1} x_{1}+\cdots+\alpha_{n} x_{n} \in X: \quad \sum_{i=1}^{n} \alpha_{i}=1 ; \quad \alpha_{i} \geq 0, x_{i} \in C \quad \text { para cada } i=1, \ldots, n\right\} .
$$

Ou seja, a envoltória convexa de $C$ é o conjunto de todas as combinações convexas de um número finito de elementos de $C$.

Definição 1.8. Seja $(X, d)$ um espaço vetorial métrico, dizemos que d é invariante, se $d(x+a, y+a)=d(x, y)$ para todo $x, y, a \in X$.

Seja $(X, d)$ um espaço vetorial métrico, definimos para cada $x_{0} \in X$ e $r>0$ a bola aberta centrada em $x_{0}$ e rádio $r$ como o conjunto $\mathfrak{B}_{r}\left(x_{0}\right)=\left\{x \in X, \quad d\left(x_{o}, x\right)<r\right\}$, em particular $\mathfrak{B}_{1}(0)$ é a bola aberta unitária e $\mathfrak{B}_{X}=\{x \in X, \quad d(0, x) \leq 1\}$ é a bola fechada unitária. 
Num espaço métrico a topologia gerada pela métrica ao considerar um aberto como união de bolas abertas, é chamada de topologia induzida pela métrica.

Seja $(X, \tau)$ um espaço topológico, se a topologia $\tau$ é gerada por uma métrica $d$, então dizemos que $\tau$ e $d$ são compatíveis.

Seja $X$ um espaço normado. A métrica $d$ induzida pela norma é definida por $d(x, y)=$ $\|x-y\|$. A topologia em $X$ obtida pela métrica induzida será chamada de topologia gerada pela norma.

Definição 1.9. Dizemos que um espaço topológico $X$ é normalizável se existe uma norma em $X$ tal que a topologia em $X$ coincide com a topologia gerada pela norma.

Um espaço topológico $X$ é chamado Hausdorff, se para todo par de elementos distintos $x, y \in X$ existem abertos $U$ e $V$, contendo a $x$ e $y$, respetivamente, tais que $U \cap V=\emptyset$.

Vamos denotar por $\bar{M}$ e $M^{\mathrm{o}}$ o fecho e o interior, respetivamente, de um subconjunto $M$ de um espaço topológico $X$.

Definição 1.10. Um subconjunto $M$ de um espaço topológico $X$ é chamado magro em $X$ se $(\bar{M})^{\circ}=\emptyset$

Definição 1.11. Um espaço topológico é de Baire, se não é união enumerável de conjuntos magros.

Teorema 1.12 (Baire). Todo espaço métrico completo, não vazio, é de Baire.

Demonstração. [Meg98].

Seja $X$ um espaço normado, vamos denotar por $X^{*}$ o espaço dual de $X$, isto é, o espaço (de Banach) de todas as aplicações lineares contínuas $f: X \rightarrow \mathbb{K}$, munido com a norma $\|f\|=\sup _{x \in \mathfrak{B}_{X}}|f(x)|$ para $f \in X^{*}$. Denotamos por $X^{* *}$ o espaço dual de $X^{*}$.

Definição 1.13. Sejam $X$ e $Y$ espaços vetoriais normados. Se $T: X \rightarrow Y$ é uma aplicação linear contínua então o operador transposto de $T$ é o operador $T^{*}: Y^{*} \rightarrow X^{*}$, definido por $\left(T^{*}(g)\right)(x)=g(T(x))$, para todo $g \in Y^{*}$ e todo $x \in X$.

Lema 1.14. Seja $T: X \rightarrow Y$ é uma aplicação linear contínua entre espaços normados $X$ e $Y$. Então o operador transposto de $T$ é contínuo e $\left\|T^{*}\right\|=\|T\|$.

Demonstração. [Meg98]. 
Teorema 1.15 (Hahn-Banach). Seja X um espaço vetorial normado sobre $\mathbb{K}$. Seja $M$ um subespaço de $X$ e seja $g$ uma função a valores reais sobre $X$ com as seguintes propriedades:

1. $g(x+y) \leq g(x)+g(y)$ para todo $x, y \in X$;

2. $g(\alpha x)=|\alpha| g(x)$ para todo $\alpha \in \mathbb{K}$ e $x \in X$.

Se $f: M \rightarrow \mathbb{R}$ é uma aplicação linear contínua sobre $M$ tal que $|f(m)| \leq g(m)$ para todo $m \in M$, então existe um funcional $F \in X^{*}$ tal que $F(x)=f(x)$ para cada $x \in M e$ $|F(x)| \leq g(x)$ para todo $x \in X$.

Para cada $x_{0} \in X$ a aplicação $Q\left(x_{0}\right): X^{*} \rightarrow \mathbb{K}$ dada por $Q\left(x_{0}\right)(f)=f\left(x_{0}\right)$, para todo $f \in X^{*}$, é uma aplicação linear contínua tal que $\left\|Q\left(x_{0}\right)\right\|=\left\|x_{0}\right\|$. Logo a função $Q: X \rightarrow X^{* *}$, que a cada $x_{0} \in X$ associa $Q\left(x_{0}\right)$ como antes, é uma isometria linear sobre um subespaço de $X^{* *}$.

Definição 1.16. O espaço normado $X$ é chamado reflexivo se a aplicação $Q$ é sobrejetora.

Dado um subconjunto $M$ de um espaço normado $X, M^{\perp}$ denotará o conjunto de funcionais lineares $f \in X^{*}$ tais que $f(m)=0$ para todo $m \in M . M^{\perp}$ é chamado de anulador de M. $M^{\perp \perp} \subseteq X^{* *}$ representa o anulador de $M^{\perp}$.

Seja $X$ um espaço normado e $M$ um subespaço fechado de $X$. Denotaremos por $X / M$ o espaço normado quociente $\{x+M: x \in X\}$ com a norma $\|x+M\|=\inf \{\|x-m\|: m \in M\}$.

Lema 1.17. Seja $M$ um subespaço de um espaço vetorial normado X. Então

(a) $X^{*} / M^{\perp}$ é isométrico a $M^{*}$ pela aplicação $S: X^{*} / M^{\perp} \rightarrow M^{*}$ definida por $S\left(f+M^{\perp}\right)=$ $\left.f\right|_{M}$, onde $f+M^{\perp} \in X^{*} / M^{\perp}$ e $\left.f\right|_{M}$ é a restrição de f a $M$.

(b) Se $M$ é fechado (e então $X / M$ é um espaço vetorial normado) então $(X / M)^{*}$ é isométrico a $M^{\perp}$ pela aplicação $T: M^{\perp} \rightarrow(X / M)^{*}$ definida por $T\left(m^{\perp}\right)(x+M)=m^{\perp}(x)$.

Demonstração. [Meg98].

Lema 1.18. Sejam $X$ um espaço normado e $M$ um subespaço de $X$. Se $Q$ é a aplicação canônica de $X$ em $X^{* *}$, então $M$ é reflexivo se, e somente se, $Q(M)=M^{\perp \perp}$.

Demonstração. [Meg98].

Definição 1.19. Seja $X$ um espaço normado

A topologia fraca $(w)$ em $X$ é a topologia gerada pela base formada pelos conjuntos

$$
\mathcal{O}=\left\{x \in X ;\left|f_{i}\left(x-x_{0}\right)\right|<\epsilon \quad \text { para } \quad i=1, \ldots, n\right\}
$$


para todas as escolhas de $x_{0} \in X, f_{1}, \ldots, f_{n} \in X^{*}$ e $\epsilon>0$.

Analogamente, a topologia fraca estrela $\left(w^{*}\right)$ no espaço dual $X^{*}$ de $X$ é generada pelos conjuntos

$$
\mathcal{O}^{*}=\left\{f \in X^{*} ;\left|\left(f-f_{0}\right)\left(x_{i}\right)\right|<\epsilon \quad \text { para } i=1, \ldots, n\right\}
$$

para todas as escolhas de $f_{0} \in X^{*}, x_{1}, \ldots, x_{n} \in X$ e $\epsilon>0$.

Lema 1.20. Seja X um espaço normado.

(a) Sejam $f, f_{1}, f_{2} \ldots \in X^{*}$. Então $f_{n} \stackrel{\mathrm{w}^{*}}{\longrightarrow} f$ se, e somente se, $\lim _{n \rightarrow \infty} f_{n}(x)=f(x)$ para todo $x \in X$.

(b) Sejam $x, x_{1}, x_{2} \ldots \in X$. Então $x_{n} \stackrel{\mathrm{w}}{\rightarrow} x$ se, e somente se, $\lim _{n \rightarrow \infty} f\left(x_{n}\right)=f(x)$ para todo $f \in X^{*}$.

Demonstração. Ver [Fea01]

Teorema 1.21 (Mazur). Seja X um espaço de Banach e $E \subseteq X$ un subconjunto convexo de $X$. Então E é fechado em relação à topologia fraca se, e somente se, E é fechado em relação à topologia da norma.

Demonstração. Ver [Fea01]

Teorema 1.22 (Aplicação aberta). Sejam $X$ e $Y$ espaços de Banach. Se $T: X \rightarrow Y$ é um operador linear contínuo sobrejetor, então $T$ é uma aplicação aberta, isto é, T leva qualquer conjunto aberto sobre um conjunto aberto.

Definição 1.23. Seja $X$ um espaço de Banach complexo, o conjugado $\bar{X}$ de $X$ é definido como o espaço $X$ munido com a lei escalar $\lambda \odot x=\bar{\lambda} x$ em vez de $\lambda x$. Assim o conjugado de $X$ é sempre $\mathbb{R}$-linearmente isométrico a $X$.

\subsection{Espaços vetoriais topológicos}

Um espaço vetorial topológico é um espaço vetorial $X$ sobre um corpo $\mathbb{K}=\mathbb{R}$ ou $\mathbb{C}$, munido com uma topologia $\tau$ tal que

1. cada ponto de $X$ é um conjunto fechado;

2. as operações do espaço vetorial são contínuas em relação a $\tau$

Isto é, para cada $x \in X$ o conjunto $X-\{x\}$, complementar de $x$, é aberto, e as operações:

$$
X \times X \ni(x, y) \rightarrow x+y \in X ; \quad \mathbb{K} \times X \ni(k, x) \rightarrow k x \in X
$$


são contínuas, onde os espaços $X \times X$ e $\mathbb{K} \times X$ estão munidos da topologia produto.

É claro que as funções

$$
T_{a}(x)=x+a, a \in X ; \quad M_{\lambda}(x)=\lambda x, \lambda \in \mathbb{K}, \quad \lambda \neq 0
$$

são homeomorfismos de $X$. Assim, um conjunto $E$ é aberto se e somente se, $E+a$ é aberto. Em consequência, para determinar $\tau$ é suficiente definir as vizinhanças de 0 . Dizemos que $\mathcal{U}$ é uma base local de 0 , ou simplesmente uma base local, se cada $U \in \mathcal{U}$ é um aberto contendo 0 , e cada vizinhança de 0 contem um elemento de $\mathcal{U}$.

Lema 1.24. Todo espaço vetorial topológico é Hausdorff. Logo, se $\mathcal{U}$ é uma base local, então $\bigcap \mathcal{U}=\{0\}$

Demonstração. [Rud73].

Definição 1.25. Seja $(X, \tau)$ um espaço vetorial topológico e E um subconjunto de $X$, então

(a) E é convexo se quaisquer que sejam $x, y \in E$, e $t \in[0,1]$, tem-se $t x+(1-t) y \in E$.

(b) E é limitado se para cada vizinhança $U$ de 0 , existe $t>0$ tal que $E \subseteq s U$ para todo $s>t$.

(c) E é equilibrado se quaisquer que sejam $\lambda \in \mathbb{K}$ com $|\lambda| \leq 1$, e $x \in E$, tem-se que $\lambda x \in E$

(d) E é absorvente se para cada $x \in X$, existe $t=t(x)>0$ tal que $x \in t E$

As seguintes propriedades dos anteriores tipos de conjuntos são simples de provar. Para os detalhes sugerimos o livro de Rudin [Rud73].

\section{Lema 1.26.}

(a) O interior e o fecho de um conjunto convexo são convexos.

(b) O fecho da soma de dois conjuntos contém a soma dos fechos destes dois conjuntos.

(c) O fecho de um conjunto equilibrado é equilibrado.

(d) Se o interior de um conjunto equilibrado contém 0, então ele é equilibrado.

(e) A soma e união finita de conjuntos limitados é limitado.

Definição 1.27. Seja $(X, \tau)$ um espaço vetorial topológico

(a) $X$ é localmente convexo se existir uma base local $\mathcal{U}$ de conjuntos convexos.

(b) X é localmente limitado se 0 possui uma vizinhança limitada. 
(c) X é metrizavél se $\tau$ é gerada por alguma métrica $d$.

(d) X é F-espaço se a topologia é induzida por uma métrica completa invariante d.

(e) X é um espaço de Fréchet se X é um F-espaço localmente convexo.

Lema 1.28. Seja X um espaço vetorial topológico

(a) Toda vizinhança de 0 contém uma vizinhança equilibrada de 0.

(b) Toda vizinhança convexa de 0 contém uma vizinhança convexa equilibrada de 0.

Demonstração. (a) Seja $U$ uma vizinhança de $0 \mathrm{em} X$. Pela continuidade da multiplicação por escalar, existe $\delta>0$ e uma vizinhança $V$ de 0 , tal que para todo $\alpha$ com $|\alpha|<\delta$ temos $\alpha V \subseteq U$. Escrevamos $W=\bigcup_{|\alpha|<\delta} \alpha V$, assim $W$ é uma vizinhança de 0 contida em $U$ e é equilibrada, pois $|\lambda| \leq 1$ implica que $\lambda \alpha V \subseteq W$ se $|\alpha|<\delta$.

(b) Seja $U$ uma vizinhança convexa de 0 . Definamos $A=\bigcap \alpha U$, onde a interseç̧ão é tomada sobre todos os escalares $\alpha$ de valor absoluto 1 . Pela parte (a) seja $W$ uma vizinhança equilibrada de 0 . Observemos que $\alpha^{-1} W \subseteq W$ se $|\alpha|=1, \log$ o $W \subseteq \alpha W \subseteq W$ e assim $\alpha^{-1} W=W$. Portanto $W \subseteq \alpha U$ para todo $|\alpha|=1$, isto é, $W \subseteq A$. Assim o interior de $A\left(A^{\circ}\right)$ é uma vizinhança de $0 . A$ é convexo pois é interseç̧ão de convexos, e pelo lema (1.26), $A^{\mathrm{o}}$ é convexo. Falta observar que $A^{\mathrm{o}}$ é equilibrado, para isto é suficiente mostrar que $A$ é equilibrado. Seja $\lambda \in \mathbb{K}$ tal que $|\lambda| \leq 1$, então $\lambda=r \beta$ onde $0 \leq r \leq 1$ e $|\beta|=1$

$$
r \beta A=\bigcap_{|\alpha|=1} r \beta \alpha U=\bigcap_{|\alpha|=1} r \alpha U
$$

agora, $\alpha U$ é convexo e contem 0 , $\log r \alpha U \subseteq \alpha U$, e assim $\lambda A \subseteq A$,com isto $A$ é equilibrado.

Observação 1.29. Todo espaço vetorial topológico possui uma base local de conjuntos equilibrados.

Todo espaço vetorial topológico localmente convexo possui uma base local de conjuntos convexos e equilibrados.

Lema 1.30. Seja $X$ um espaço vetorial topológico e $V$ uma vizinhança de 0.

(a) Se $0<r_{1}<r_{2}<\cdots$ e $r_{n} \rightarrow \infty$ quando $n \rightarrow \infty$, então

$$
X=\bigcup_{n=1}^{\infty} r_{n} V
$$

(b) Se $s_{1}>s_{2}>\cdots$ e $s_{n} \rightarrow 0$ quando $n \rightarrow \infty$ eV é limitada, então a colecção de conjuntos

$$
\left\{s_{n} V: n=1,2, \ldots\right\}
$$

é uma base local de 0. 
Demonstração. (a). Fixemos $x \in X$. Resulta da continuidade da aplicação $M_{x}: \mathbb{K} \rightarrow X$ definida por $M_{x}(\lambda)=\lambda x$ que existe uma vizinhança $W$ de 0 em $\mathbb{K}$ tal que $M_{x}(W) \subseteq V$. Portanto, para todo $n$ suficientemente grande $1 / r_{n} \in W$, assim $\left(1 / r_{n}\right) x \in V$ ou $x \in r_{n} V$ para todo $n$ exceto um número finito.

(b). Seja $U$ uma vizinhança de 0 em $X$. Se $V$ é limitada, existe $t>0$ tal que $V \subseteq s U$ para todo $s>t$. Se $n$ é suficientemente grande tal que $t s_{n}<1$, temos $V \subseteq\left(1 / s_{n}\right) U$. Portanto $U$ contém todos, exceto um número finito dos conjuntos $s_{n} V$.

Pela parte (a) do lema anterior segue-se que toda vizinhança de 0 é absorvente.

Vamos estabelecer uma condição necessária e suficiente para que um espaço vetorial topológico seja normalizável, isto é, que a topologia seja induzida por una norma.

Considere $E$ um subconjunto absorvente de $X$, o funcional de Minkowski $\mu_{E}$ de $E$ está definido por

$$
\mu_{E}(x)=\inf \left\{t>0: t^{-1} x \in E\right\} \quad(x \in X) .
$$

É claro que $\mu_{E}(x)<\infty$ para todo $x \in X$.

Lema 1.31. Seja E um conjunto convexo, equilibrado e absorvente num espaço vetorial topológico $X$. Então

(a) $\mu_{E}(x+y) \leq \mu_{E}(x)+\mu_{E}(y)$

(b) $\mu_{E}(t x)=t \mu_{E}(x), t \geq 0$

(c) $\mu_{E}(\alpha x)=|\alpha| \mu_{E}(x), \alpha \in \mathbb{K}$

Demonstração. Para cada $x \in X$, defina o conjunto

$$
H_{E}(x)=\left\{t>0: t^{-1} x \in E\right\}
$$

$H_{E}(x) \neq \emptyset$ pois $A$ é absorvente. Suponha $t \in H_{E}(x)$ e $s>t$, dado que $0 \in E$ e que $E$ é convexo, então $\left.s \in H_{E}(x)\left(s^{-1} x=s^{-1} t\left(t^{-1} x\right)+\left(1-s^{-1} t\right) 0\right) \in E\right)$. Seja $s>\mu_{E}(x)$ e $t>\mu_{E}(y)$, escrevamos $u=s+t$. Assim, $s^{-1} x \in E$ e $t^{-1} y \in E$ e de novo pela convexidade de $E$

$$
u^{-1}(x+y)=\left(\frac{s}{u}\right)\left(s^{-1} x\right)+\left(\frac{t}{u}\right)\left(t^{-1} y\right) \in E
$$

$\operatorname{logo} \mu_{E}(x+y) \leq u$, o que prova (a). A propiedade (b) é clara a partir da definição.

Seja agora, $\alpha \in K, \alpha=r \beta$ onde $r \geq 0$ e $|\beta|=1$. Da prova do Lema (1.28), temos que se $E$ é equilibrado, então $\beta E=E$, isto mostra que $\mu_{E}(\beta x)=\mu_{E}(x)$. Concluímos usando (b). que $\mu_{E}(\alpha x)=r \mu_{E}(x)=|\alpha| \mu_{E}(x)$.

Teorema 1.32. Um espaço vetorial topológico $X$ é normalizável se, e somente se, 0 possui uma vizinhança aberta convexa e limitada. 
Demonstração. Se existe uma norma $\|$.$\| compatível com a topología em X$, então a bola aberta unitária $\mathfrak{B}_{1}(0)$ é convexa e limitada.

Reciprocamente, seja $U$ uma vizinhança de 0 limitada e convexa. Pelo Lema (1.28), $U$ contém uma vizinhança $V$ de 0 convexa e equilibrada. Definamos

$$
\|x\|=\mu_{V}(x) \quad(x \in X)
$$

É claro que $V$ é limitada, logo por Lema (1.30)-(b), os conjuntos $r V(r>0)$ formam uma base local para a topologia de $X$. Se $x \neq 0$ então $x \notin r V$ para algum $r>0(X$ é Hausdorff) e assim $\|x\| \geq r$, isto prova, junto com o Lema (1.31), que $\|$.$\| define uma norma.$

Dado que $V$ é um aberto,

$$
\{x:\|x\|<r\}=r V \quad r>0
$$

em consequência a topologia da norma coincide com a topologia de $X$.

Agora observemos que o teorema da aplicação aberta é válida para $F$-espaços.

Teorema 1.33 (Teorema da aplicação aberta). Sejam $X, Y$ F-espaços e $T: X \rightarrow Y$ uma aplicação linear contínua sobrejetora. Então T é aberta.

Demonstração. Vamos mostrar que se $V$ é uma vizinhança de 0 em $X$, então $T(V)$ contém uma vizinhança de 0 em $Y$. Seja $d$ a métrica completa invariante em $X$ que é compatível com a topologia. Se $r>0$ é tal que $\mathfrak{B}_{r}(0) \subseteq V$, definamos $V_{n}=\mathfrak{B}_{2^{-n} r}(0)(n=1,2, \ldots)$. Vamos encontrar uma vizinhança $W$ de 0 em $Y$ tal que

$$
W \subseteq \overline{T\left(V_{1}\right)} \subseteq T(V)
$$

Dado que $V_{2}-V_{2} \subseteq V_{1}$, temos pelo Lema (1.26)

$$
\overline{T\left(V_{2}\right)}-\overline{T\left(V_{1}\right)} \subseteq \overline{T\left(V_{2}\right)-T\left(V_{2}\right)} \subseteq \overline{T\left(V_{1}\right)}
$$

Portanto, para provar a primeira inclusão em (1.1) é suficiente mostrar que $T\left(\overline{T\left(V_{2}\right)}\right)$ tem interior não vazio. Segue do Lema (1.30)-(a) que $X=\bigcup_{k=1}^{\infty} k V_{2}$. Logo

$$
Y=\bigcup_{k=1}^{\infty} k \overline{T\left(V_{2}\right)}
$$

Pelo teorema de Baire (1.12) existe $k_{0} \in \mathbb{N}$ tal que $\left(k_{0} \overline{T\left(V_{2}\right)}\right)^{\circ} \neq \emptyset$, dado que $x \rightarrow k_{o} x$ é um homeomorfismo de $X$, temos $\left(\overline{T\left(V_{2}\right)}\right)^{\circ} \neq \emptyset$. Notemos que o mesmo argumento mostra que $\overline{T\left(V_{n}\right)}$ é uma vizinhança de 0 para todo $n \geq 1$.

Para mostrar a segunda inclusão em (1.1), fixemos $y_{1} \in \overline{T\left(V_{1}\right)}$. Por indução vamos escolher 
$y_{n} \in \overline{T\left(V_{n}\right)}$ e $x_{n} \in V_{n}$. Suponha que $y_{n}$ já foi escolhido. Observemos que

$$
\left(y_{n}-\overline{T\left(V_{n+1}\right)}\right) \cap T\left(V_{n}\right) \neq \emptyset
$$

pois $y_{n}-\overline{T\left(V_{n+1}\right)}$ é uma vizinhança de $y_{n}$. Portanto, existe $x_{n} \in V_{n}$ tal que

$$
T\left(x_{n}\right)=y_{n}-\overline{T\left(V_{n+1}\right)}
$$

Definamos $y_{n+1}=y_{n}-T\left(x_{n}\right)$. Com isto, $y_{n+1} \in \overline{T\left(V_{n+1}\right)}$ e podemos continuar o processo. Dado que $d\left(x_{n}, 0\right)<2^{-n} r(n \geq 1)$, então as somas $s_{n}=x_{1}+\cdots+x_{n}$ formam uma sequência de Cauchy. Com efeito, se $n<m$

$$
d\left(s_{m}, s_{n}\right)=d\left(s_{m}-s_{n}, s_{n}-s_{n}\right)=d\left(\sum_{k>n}^{m} x_{k}, 0\right) \leq\left(\sum_{k>n}^{m} 2^{-k}\right) r
$$

e em consequência, $d\left(s_{m}, s_{n}\right) \rightarrow 0$ quando $n, m \rightarrow \infty$. Como $X$ é completo, seja $x \in X$ o limite da sequência $\left(s_{n}\right)_{n}$. Observemos que

$$
\sum_{n=1}^{m} T x_{n}=\sum_{n=1}^{m}\left(y_{n}-y_{n+1}\right)=y_{1}-y_{n+1} \text {. }
$$

Segue da continuidade de $T$ que $\lim _{n \rightarrow \infty} y_{n+1}=0$, pois $y_{n+1} \in \overline{T\left(V_{n+1}\right)}$. Concluímos que $y_{1}=T x \in T(V)$. O que completa a demonstração.

Corolario 1.34. Sejam $X, Y$ F-espaços e $T: X \rightarrow Y$ um operador linear continuo sobrejetor. Então $X / \operatorname{ker} T$ é isomorfo a $Y$.

Demonstração. A aplicação $S: X / \operatorname{ker} T \rightarrow Y$, dada por $S(x+\operatorname{ker} T)=T(x)$ é bijetora e contínua entre $F$-espaços. Pelo teorema da aplicação aberta, a inversa de $S$ é contínua, portanto $S$ é um isomorfismo.

\subsection{Espaços Quase-normados}

Consideremos $X$ um espaço vetorial topológico localmente limitado, e seja $V$ uma vizinhança equilibrada e limitada de 0 , fazendo analogia ao Teorema (1.32), estudemos o funcional de Minkowski $\mu_{V}$ associado a $V$. Neste caso, $\mu_{V}$ não sempre é uma norma, mas satisfaz as seguintes propriedades:

(Q1) $\mu_{V}(x) \geq 0$ para todo $x \in X$,

(Q2) Se $\mu_{V}(x)=0$, então $x=0$,

(Q3) $\mu_{V}(\alpha x)=|\alpha| \mu_{V}(x)$ para todo $\alpha \in \mathbb{K}$ e $x \in X$, 
(Q4) Existe $K \geq 1$ tal que $\mu_{V}(x+y) \leq K\left(\mu_{V}(x)+\mu_{V}(y)\right)$ para todo $x, y \in X$

(Q1) é claro por definição e (Q2) segue do fato que $\bigcap_{\alpha>0} \alpha V=\{0\}$. (Q3) tem a mesma prova que no Lema (1.31).

Dado que $V+V$ é limitada, existe $t>0$ tal que $V+V \subseteq s V$ para todo $s>t$. Vamos considerar $K>0$ uma constante tal que $\bigcup_{\lambda \in[0,1]}(\lambda V+(1-\lambda) V) \subseteq K V$. Sejam $s>\mu_{V}(x)$ e $t>\mu_{V}(y)$ então $x / s$ e $y / t$ pertencem a $V$, o que implica

$$
\frac{s}{s+t} \frac{x}{s}+\frac{t}{s+t} \frac{y}{t}=\frac{1}{s+t}(x+y) \in K V
$$

portanto $x+y \in K(s+t) V$ e em consequência

$$
\mu_{V}(x+y) \leq K\left(\mu_{V}(x)+\mu_{V}(y)\right)
$$

o que prova (Q4). Observamos que se $V$ é convexa, a melhor constante é $K=1$, e nesse caso obtemos uma norma, tal como foi estabelecido no Teorema (1.32)

Agora observemos que a topologia em $X$ pode ser determinada usando $\mu_{V}$. Isto, porque $E=\left\{x \in X: \mu_{V}(x) \leq 1\right\}=V$ e os conjuntos $\alpha V, \alpha>0$ formam uma base de de vizinhanças de 0 .

Uma aplicação que satisfaz as propriedades (Q1) até (Q4) é chamada de quase-norma. Uma constante $K$ em (Q4) chama-se módulo de concavidade.

Temos mostrado até aqui, que

Lema 1.35. A topologia de um espaço localmente limitado pode ser determinada por uma quase-norma.

Também é válida a afirmação recíproca:

Lema 1.36. Seja $X$ um espaço vetorial e \|.\| uma quase-norma em X. Então X é localmente limitado com a topologia induzida por \|.\|

Demonstração. Consideremos $E=\{x \in X:\|x\| \leq 1\}$. A coleção $r E, r>0$, forma uma base local de 0, de uma topologia Hausdorff, pois $\bigcap_{\alpha>0} \alpha E=\{0\}$. Segue-se do fato $\frac{1}{2 K} E+\frac{1}{2 K} E \subseteq$ $E$ (por Q4), que a topologia é compatível com a estrutura linear. Claramente $E$ é limitado, o que completa a prova.

Definição 1.37. Um espaço vetorial topológico chama-se quase normado se ele é localmente limitado, o que é equivalente a dizer que a topologia é gerada por uma quase-norma.

Observação 1.38. Num espaço localmente limitado a quase-norma não é necessáriamente uma função contínua em relação à topologia que gera. Assim, por exemplo, se $\left(\alpha_{n}\right)_{n}$ é uma sequência de escalares convergente para um $\alpha \in \mathbb{K}$ e $x \in X$ tal que

$$
\left\|\alpha_{n} x\right\| \leq C, \quad n \in \mathbb{N}
$$


para alguma constante $C>0$, então

$$
\begin{aligned}
\|\alpha x\| & \leq K\left(\left\|\alpha x-\alpha_{n} x\right\|+\left\|\alpha_{n} x\right\|\right) \\
& \leq K\left|\alpha-\alpha_{n}\right|\|x\|+K C
\end{aligned}
$$

portanto, $\|\alpha x\| \leq K C$, onde $K$ é um módulo de concavidade da quase-norma.

De forma análoga ao caso de espaços normados, se $X$ é um espaço quase-normado e $M$ é um subespaço fechado de $X$, então a topologia de $X / M$ é determinada pela quase-norma

$$
\|u\|=\inf \{\|x\|: \pi(x)=u\}
$$

onde $\pi: X \rightarrow X / M$ é a aplicação quociente. Com frequência usaremos a seguinte observação: seja $x \in X$, nas condições anteriores, dado $\epsilon>0$ existe $y \in X$ com $x+M=y+M$ tal que $\|y\|<\|x+M\|+\epsilon$.

Se $T: X \rightarrow Y$ é uma aplicação linear entre dois espaços quase-normados, observamos facilmente, que $T$ é contínua se

$$
\|T\|=\sup \{\|T x\|:\|x\| \leq 1\}<\infty
$$

Definição 1.39. Duas quase-normas |.| e $\|$.$\| em X$ são equivalentes se existem constantes $0<m \leq M<\infty$ tais que

$$
m|x| \leq\|x\| \leq M|x|, \quad x \in X
$$

Definição 1.40. Seja $0<p \leq 1$. Um subconjunto $C$ de um espaço vetorial $X$ sobre $\mathbb{K} e ́$ $p$-convexo se para todo $x, y \in C, \alpha x+\beta y \in C$ sempre que $\alpha \geq 0, \beta \geq 0$ e $\alpha^{p}+\beta^{p}=1$. $O$ conjunto $C$ é absolutamente $p$-convexo se $\alpha x+\beta y \in C$, sempre que $x, y \in C$ e $\alpha, \beta \in K$ com $|\alpha|^{p}+|\beta|^{p} \leq 1$

Definição 1.41. Uma quase-norma $\|$.$\| é p-subaditiva, 0<p \leq 1$, se $\|x+y\|^{p} \leq\|x\|^{p}+\|y\|^{p}$ para todo $x, y \in X$.

Definição 1.42. Um espaço $X$ localmente limitado é localmente p-convexo (ou simplesmente p-convexo), $0<p \leq 1$, se possui uma vizinhança limitada absolutamente $p$-convexa de 0 .

Agora vamos estabelecer o teorema de Aoki-Rolewicz: se $X$ é um espaço localmente limitado então $X$ é $p$-convexo para algum $p$. Em termos de quase-normas(a demonstração segue [KPR84] ):

Teorema 1.43 (Aoki-Rolewicz(1957)). Seja (X,\|.\|) um espaço quase-normado. Então existem números positivos $p, L$ e uma quase-norma p-subaditiva |||.||| equivalente a $\|$.$\| , tais que$

$$
\left\|\left|\sum_{i=1}^{n} x_{i}\right|\right\| \leq L\left(\sum_{i=1}^{n}\|\| x_{i}\|\|^{p}\right)^{1 / p}
$$


para todo $n$ e $x_{1}, \ldots, x_{n} \in X$.

Demonstração. Seja $K$ um módulo de concavidade de $\|\cdot\|$, escrevamos $\rho=2 K$, assim $\left\|x_{1}+x_{2}\right\| \leq \rho \max \left\{\left\|x_{1}\right\|,\left\|x_{2}\right\|\right\}$ para todo $x_{1}, x_{2} \in X$. Seja $0<p \leq 1$ tal que $2^{1 / p}=\rho$. Definamos:

$$
\|\| x \|=\inf \left\{\left(\sum_{i=1}^{n}\left\|x_{i}\right\|^{p}\right)^{1 / p}: \sum_{i=1}^{n} x_{i}=x\right\}
$$

Então $|\|x\|| \leq\|x\|, \operatorname{logo}\||x \||$ satisfaz (Q1),(Q2) e (Q3). Vejamos que $|\|. \mid\|$ é $p$-subaditiva. Dado $\epsilon>0$, sejam $\sum_{i=1}^{n} x_{i}=x$ e $\sum_{j=1}^{m} y_{j}=y$ tais que

$$
\begin{aligned}
& \|\mid x\|^{p}+\epsilon>\sum_{i=1}^{n}\left\|x_{i}\right\|^{p} \\
& \|y\|\left\|^{p}+\epsilon>\sum_{j=1}^{m}\right\| y_{j} \|^{p}
\end{aligned}
$$

$\operatorname{Logo}\|\| x+y\left\|^{p} \leq \sum\left(\left\|x_{i}\right\|^{p}+\left\|y_{j}\right\|^{p}\right)<\right\|\|x\|^{p}+\|\| y \|\left.\right|^{p}+2 \epsilon$. Como $\epsilon$ era arbitrário, concluímos que $\|x+y\|\left\|^{p} \leq\right\|\|x\|^{p}+\|\| y\|\|^{p}$.

Para provar (Q4), vamos demonstrar os seguintes dois fatos:

FATO 1. $\left\|x_{1}+\cdots+x_{n}\right\| \leq \max _{1 \leq k \leq n} \rho^{k}\left\|x_{k}\right\|$, para todo $n \in \mathbb{N}$.

A demonstração é por indução sobre $n$. O caso $n=2$ é claro. Suponhamos que a fórmula é valida para $n>2$, então

$$
\begin{aligned}
\left\|x_{1}+\cdots+x_{n+1}\right\| & \leq \rho \max \left\{\left\|x_{i}\right\|,\left\|x_{2}+\cdots+x_{n+1}\right\|\right\} \\
& \leq \rho \max \left\{\left\|x_{i}\right\|, \max _{1 \leq k \leq n} \rho^{k}\left\|x_{k+1}\right\|\right\} \\
& =\max _{1 \leq k \leq n+1} \rho^{k}\left\|x_{k}\right\|
\end{aligned}
$$

isto completa o passo indutivo.

FATO 2. $\left\|x_{1}+\cdots+x_{n}\right\| \leq 4^{1 / p}\left(\sum_{i=1}^{n}\left\|x_{i}\right\|^{p}\right)^{1 / p}$, para todo $n \in \mathbb{N}$.

Definamos a função $H: X \rightarrow \mathbb{R}$ por

$$
H(x)=\left\{\begin{array}{rll}
2^{n / p} & \text { se } & 2^{n-1 / p}<\|x\| \leq 2^{n / p}, n \in \mathbb{Z} \\
0 & \text { se } \quad x=0
\end{array}\right.
$$

Logo $\|x\| \leq H(x) \leq 2^{1 / p}\|x\|$. Vamos mostrar por indução que

$$
\left\|x_{1}+\cdots+x_{n}\right\| \leq 2^{1 / p}\left(H\left(x_{1}\right)^{p}+\cdots+H\left(x_{n}\right)^{p}\right)^{1 / p}
$$


e em consequência

$$
\begin{aligned}
\left\|x_{1}+\cdots+x_{n}\right\| & \leq 2^{1 / p}\left(2\left\|x_{1}\right\|^{p}+\cdots+2\left\|x_{n}\right\|^{p}\right) \\
& =4^{1 / p}\left(\left\|x_{1}\right\|^{p}+\cdots+\left\|x_{n}\right\|^{p}\right)
\end{aligned}
$$

A fórmula (1.2) é clara para $n=1$. Suponhamos que também é válida para $n=m$. Sejam $x_{1}, \ldots, x_{m+1} \in X$. Podemos supor $\left\|x_{1}\right\| \geq\left\|x_{2}\right\| \geq \cdots \geq\left\|x_{m+1}\right\|$. Vamos considerar dois casos. O primeiro, se os elementos do conjunto $\left\{H\left(x_{i}\right): 1 \leq i \leq m+1\right\}$ são todos distintos. Neste caso, $2^{1 / p} H\left(x_{j+1}\right) \leq H\left(x_{j}\right)$ para $j=1, \ldots, m$. Logo, $H\left(x_{1}\right) \geq 2^{i-1 / p} H\left(x_{i}\right)$. Agora,

$$
\rho^{i}\left\|x_{i}\right\| \leq 2^{i / p} H\left(x_{i}\right) \leq 2^{1 / p} H\left(x_{1}\right)
$$

e pelo FATO 1:

$$
\begin{aligned}
\left\|x_{1}+\cdots+x_{m+1}\right\| & \leq 2^{1 / p} H\left(x_{1}\right) \\
& \leq 2^{1 / p}\left(H\left(x_{1}\right)^{p}+\cdots+H\left(x_{m+1}\right)^{p}\right)^{1 / p}
\end{aligned}
$$

O segundo caso é quando $H\left(x_{j}\right)=H\left(x_{j+1}\right)$ para algum $j, 1 \leq j \leq m$. Então para algum $r \in \mathbb{Z}$

$$
2^{r-1 / p}<\left\|x_{j+1}\right\| \leq\left\|x_{j}\right\| \leq 2^{r / p}
$$

Portanto, $H\left(x_{j}+x_{j+1}\right)^{p} \leq 2^{r+1}=H\left(x_{j}\right)^{p}+H\left(x_{j+1}\right)^{p}$. Concluímos aplicando a hipótese de indução:

$$
\begin{aligned}
\left\|x_{1}+\cdots+x_{m+1}\right\| & \leq 2^{1 / p}\left(\sum_{i \notin\{j, j+1\}} H\left(x_{i}\right)^{p}+H\left(x_{j}+x_{j+1}\right)^{p}\right)^{1 / p} \\
& \leq 2^{1 / p}\left(\sum_{i=1}^{m+1} H\left(x_{i}\right)^{p}\right)^{1 / p}
\end{aligned}
$$

e assim a prova do FATO 2 está completa. Este resultado implica que

$$
\left(\frac{1}{4}\right)^{1 / p}\|x\| \leq\|\| x\|\| \leq\|x\|
$$

O que permite provar (Q4):

$$
\begin{aligned}
\left\|\left|x_{1}+x_{2}\right|\right\| & \leq\left(\left\|x_{1}\right\|^{p}+\left\|x_{2}\right\|^{p}\right)^{1 / p} \\
& \leq 2^{1 / p}\left(\left\|x_{1}\right\|+\left\|x_{2}\right\|\right) \\
& \leq 2^{1 / p} 4^{1 / p}\left(\left|\left\|x_{1}\right\|\right||+|\left|x_{2}\right| \|\right) \\
& =\rho^{3}\left(\left\||| x_{1}\right\||+|\left|x_{2}\right| \|\right)
\end{aligned}
$$


Por último encontramos a constante $L>0$ :

$$
\begin{aligned}
\left\|\sum_{i=1}^{n} x_{i}\right\| \mid \leq\left\|\sum_{i=1}^{n} x_{i}\right\| & \leq 4^{1 / p}\left(\left\|x_{1}\right\|^{p}+\cdots+\left\|x_{n}\right\|^{p}\right)^{1 / p} \\
& \leq 4^{1 / p}\left(4 \left|\left\|x _ { 1 } \left|\left\|^{p}+\cdots+4\left|\left\|x_{n}\right\|\right|^{p}\right)^{1 / p}\right.\right.\right.\right. \\
& =4^{2 / p}\left(\left\||| x_{1}\left|\left\|^{p}+\cdots+\right\|\right| x_{n} \mid\right\|^{p}\right)^{1 / p}
\end{aligned}
$$

Observação 1.44. Sejam $(X,\|\|$.$) e (Y,||$.$) dois espaços quase-normados. Segue da prova$ do teorema de Aoki-Rolewicz, que podemos tomar o mesmo $p \in(0,1]$ tal que $\|$.$\| e |.| sejam$ equivalentes a quase-normas p-subaditivas. Com efeito, é suficiente tomar $K$ um módulo de concavidade comum para as quase-normas de X e $Y$ e continuar a construção feita na demonstração.

Definição 1.45. Seja X um espaço quase-normado. Consideremos pelo teorema de AokiRolewicz um $p \in(0,1]$ tal que a quase-norma em X seja equivalente a uma quase-norma \|.\| p-subaditiva. Então

$$
d(x, y)=\|x-y\|^{p}
$$

define uma métrica invariante em $X$.

$O$ espaço quase-normado $X$ é chamado de quase-Banach se $X$ é completo em relação à métrica d.

Observação 1.46. A definição anterior é independente de p pois as métricas obtidas para diferentes p são equivalentes.

Teorema 1.47. Um espaço quase-Banach $X$ é isomorfo a um espaço de Banach se, $e$ somente se, $X$ possui uma base local de vizinhanças convexas.

Demonstração. É claro que se um espaço quase-Banach é isomorfo a um espaço de Banach, então 0 possui uma base local de vizinhanças convexas. Reciprocamente, se um espaço quase-Banach $X$ possui uma vizinhança convexa de 0, o Teorema (1.32) garanta que $X$ é normalizável e em consequência é isomorfo a um espaço normado completo, ou seja, um espaço de Banach.

\subsection{Decomposição de Schauder}

Seja $X$ um espaço de Banach. Uma sequência $\left\{X_{n}\right\}_{n=1}^{\infty}$ de subespaços fechados de $X$ é chamada uma decomposição de Schauder de $X$, se cada $x \in X$ possui uma única representação da forma $x=\sum_{n=1}^{\infty} x_{n} \operatorname{com} x_{n} \in X_{n}$ para todo $n \in \mathbb{N}$. Neste caso usaremos a notação $X=\sum X_{n}$. Se $\operatorname{dim} X_{n}<\infty$ para todo $n$, então $\left\{X_{n}\right\}_{n=1}^{\infty}$ é chamada decomposição de Schauder finito-dimensional ou simplesmente F.D.D. Vamos chamar de $k$-F.D.D uma F.D.D tal 
que $\operatorname{dim} X_{n}=k$ para todo $n \in \mathbb{N}$. Em particular, uma 1-F.D.D não é outra coisa do que uma base de Schauder $\left\{x_{n}\right\}_{n=1}^{\infty}$ de $X$, onde $X_{n}=\operatorname{span}\left\{x_{n}\right\}$ para cada $n \in \mathbb{N}$.

Cada decomposição de Schauder $\left\{X_{n}\right\}_{n=1}^{\infty}$ de um espaço de Banach $X$ determina uma sequência de projeções $\left\{P_{n}\right\}_{n=1}^{\infty}$ sobre $X$, definidas por $P_{n}\left(\sum_{k=1}^{\infty} x_{k}\right)=\sum_{k=1}^{n} x_{k}$ que satisfazem o lema seguinte.

Lema 1.48. Seja $\left\{X_{n}\right\}_{n=1}^{\infty}$ uma decomposição de Schauder de um espaço de Banach $X$. Então as projeções associadas $P_{n}: X \rightarrow X$ são contínuas e $\sup _{n \in \mathbb{N}}\left\|P_{n}\right\|<\infty$.

O número $\sup _{n \in \mathbb{N}}\left\|P_{n}\right\|$ do lema anterior é chamado de constante da decomposição $\left\{X_{n}\right\}_{n=1}^{\infty}$. A prova deste lema é análoga à dada no caso 1-F.D.D. ou seja, quando temos uma base de Schauder(ver [Fea01]).

Uma decomposição de Schauder $\left\{X_{n}\right\}_{n=1}^{\infty}$ de um espaço de Banach $X$ é chamada contrátil, se para cada $x^{*} \in X^{*}$ temos $\lim _{n \rightarrow \infty}\left\|P_{n}^{*} x^{*}-x^{*}\right\|=0$. Em particular, dizemos que uma base de Schauder $\left\{x_{n}\right\}_{n=1}^{\infty}$ de um espaço de Banach $X$ é contrátil, se a decomposição $\left\{X_{n}\right\}_{n=1}^{\infty}$ é contrátil, onde $X_{n}=\operatorname{span}\left\{x_{n}\right\}$. A base de Schauder é chamada de limitadamente completa se $\sum_{n=1}^{\infty} a_{n} x_{n}$ converge para todos os escalares $a_{n}$ tais que $\sup _{k}\left\|\sum_{n=1}^{k} a_{n} x_{n}\right\|<\infty$. Um teorema clássico caracteriza a reflexividade em espaços de Banach com base de Schauder(ver [Jam50]): $X$ é reflexivo, se e somente se, a base é contrátil e limitadamente completa. O resultado pode-se extender para decomposições de Schauder finito dimensionais, em particular vamos demonstrar o seguinte teorema.

Teorema 1.49. Seja $X$ um espaço de Banach reflexivo e $X=\sum X_{n}$ uma F.D.D. Então a decomposição $\left\{X_{n}\right\}_{n=1}^{\infty}$ é contrátil.

Demonstração. Para cada $n \in \mathbb{N}$, o subespaço $X_{n}$ tem dimensão finita, logo consideremos $\left\{e_{1}^{n}, \ldots, e_{k_{n}}^{n}\right\}$ uma base de $X_{n}$. Assim, dado $x \in X, x=\sum_{n=1}^{\infty} x_{n}$ onde

$$
x_{n}=\sum_{i=1}^{k_{n}} a_{i}^{n} e_{i}^{n} \quad \text { para } \quad a_{i}^{n} \in K
$$

A aplicação $f_{i}^{n}: X_{n} \rightarrow K$ definida por $f_{i}^{n}\left(x_{n}\right)=a_{i}^{n}$ é contínua, pois $\operatorname{dim} X_{n}<\infty$. Assim o operador linear $e_{i}^{n *}: X \rightarrow K$, definido como $e_{i}^{n *}(x)=a_{i}^{n}$ é contínuo, já que

$$
\left|e_{i}^{n *}(x)\right|=\left|f_{i}^{n}\left(P_{n}-P_{n-1}\right)(x)\right| \leq\left\|f_{i}^{n}\right\| 2 \mathcal{K}\|x\|
$$

onde $\mathcal{K}$ é a constante da decomposição. 
Seja $x^{*} \in X^{*}$, então para cada $m$

$$
\begin{aligned}
P_{m}^{*}\left(x^{*}\right)(x) & =x^{*}\left(\sum_{n=1}^{m} x_{n}\right) \\
& =\sum_{n=1}^{m} x^{*}\left(\sum_{i=1}^{k_{n}} a_{i}^{n} e_{i}^{n}\right) \\
& =\sum_{n=1}^{m} \sum_{i=1}^{k_{n}} a_{i}^{n} x^{*}\left(e_{i}^{n}\right) \\
& =\sum_{n=1}^{m} \sum_{i=1}^{k_{n}} x^{*}\left(e_{i}^{n}\right) e_{i}^{n *}(x)
\end{aligned}
$$

Agora mostremos que $P_{m}^{*}\left(x^{*}\right) \stackrel{\mathrm{w}^{*}}{\longrightarrow} x^{*}$ em $X^{*}$. Com efeito para cada $x \in X$

$$
\begin{aligned}
\lim _{m \rightarrow \infty} P_{m}^{*}\left(x^{*}\right)(x) & =\lim _{m \rightarrow \infty} x^{*}\left(P_{n}(x)\right)=\lim _{m \rightarrow \infty} x^{*}\left(\sum_{k=1}^{m} x_{k}\right) \\
& =x^{*}\left(\lim _{m \rightarrow \infty} \sum_{k=1}^{m} x_{k}\right) \\
& =x^{*}(x)
\end{aligned}
$$

Portanto, usando o teorema de Mazur e a reflexividade de $X$

$$
\begin{aligned}
X^{*} & =\overline{\operatorname{span}}^{w^{*}}\left\{e_{i}^{k *}\right\} \\
& =\overline{\operatorname{span}}^{w}\left\{e_{i}^{k *}\right\} \\
& =\overline{\operatorname{span}}\left\{e_{i}^{k *}\right\}
\end{aligned}
$$

Assim, fica demonstrado que $P_{n}^{*}\left(x^{*}\right) \rightarrow x^{*}$ com a topologia da norma em $X^{*}$.

Vamos estabelecer um teorema sobre operadores contínuos em espaços de Banach com F.D.D contrátil(usamos como referência [LT77]), para isto precisamos da seguinte definição

Definição 1.50. Sejam $\left\{X_{n}\right\}_{n=1}^{\infty}$ uma decomposição de Schauder de X e $1=k_{1}<k_{2}<k_{3}<$ ... uma sequência crescente de números inteiros. Escreva $Y_{i}=X_{k_{i}} \oplus X_{k_{i}+1} \oplus \cdots \oplus X_{k_{i+1}-1}$ $(i=1,2, \ldots)$. Então a decomposição $\left\{Y_{i}\right\}_{i=1}^{\infty}$ de $X$ é chamada um 'blocking' da decomposição $\left\{X_{n}\right\}_{n=1}^{\infty}$.

Teorema 1.51. Seja $T: X \rightarrow Y$ um operador linear limitado entre espaços de Banach $X$ e Y. Sejam $\left\{B_{n}\right\}_{n=1}^{\infty}$ uma decomposição F.D.D contrátil de $X$ e $\left\{C_{n}\right\}_{n=1}^{\infty}$ uma F.D.D de $Y$. Dada $\left\{\epsilon_{i}\right\}_{i=1}^{\infty}$ uma sequência de números positivos com limite 0, existem 'blockings' $\left\{B_{i}^{\prime}\right\}_{i=1}^{\infty}$ de $\left\{B_{n}\right\}_{n=1}^{\infty}$ e $\left\{C_{i}^{\prime}\right\}_{i=1}^{\infty}$ de $\left\{C_{n}\right\}_{n=1}^{\infty}$ tal que para cada $x \in B_{i}^{\prime}$, existe um $y \in C_{i-1}^{\prime} \oplus C_{i}^{\prime}$ tal que $\|T x-y\| \leq \epsilon_{i}\|x\|$. 
Demonstração. Sejam $\left\{P_{n}\right\}$ e $\left\{Q_{n}\right\}$ as projecções associadas às decomposições de $X$ e $Y$ respectivamente. A prova do teorema está baseada nos dois passos seguintes.

PASSO 1. Para cada $\epsilon>0$ e cada $n \in \mathbb{N}$, existe $m \in \mathbb{N}$ tal que se $P_{m}(x)=0$ então $\left\|Q_{n} T x\right\| \leq \epsilon\|x\|$.

No caso contrário, existiria um $\epsilon>0$ e uma sequência $\left\{x_{k}\right\}_{k=1}^{\infty}$ em $X$ tal que $\left\|x_{k}\right\|=1$, $P_{k}\left(x_{k}\right)=0$ e $\left\|Q_{n} T x_{k}\right\| \geq \epsilon$ para todo $k \in \mathbb{N}$. Pelo teorema de Hahn Banach, seja $y_{k}^{*} \in\left(Q_{n} Y\right)^{*}$ com $\left\|y_{k}^{*}\right\|=1$ e tal que

$$
y_{k}^{*}\left(Q_{n} T x_{k}\right)=\left\|Q_{n} T x_{k}\right\| \geq \epsilon .
$$

Como a dimensão de $\left(Q_{n} Y\right)^{*}$ é finita então, passando a uma sub-sequência se for necessario, existe $\widetilde{y}^{*} \in\left(Q_{n} Y\right)^{*}$ tal que

$$
\widetilde{y}^{*}=\lim _{k \rightarrow \infty} y_{k}^{*}
$$

Também pelo teorema de Hahn Banach, estendemos a um funcional linear $y^{*} \in Y^{*}$ com $\left\|y^{*}\right\|=1$. Se $\mathcal{M}=\sup \left\|Q_{n}\right\|$, seja $N \in \mathbb{N}$ tal que para todo $k>N,\left\|\widetilde{y}^{*}-y_{k}^{*}\right\|<\epsilon / 2 \mathcal{M}\|T\|$. Logo

$$
\begin{aligned}
\left|y^{*}\left(Q_{n} T x_{k}\right)\right| & =\left|\widetilde{y}^{*}\left(Q_{n} T x_{k}\right)\right| \\
& \geq\left|y_{k}^{*}\left(Q_{n} T x_{k}\right)\right|-\left|\left(\widetilde{y}^{*}-y_{k}^{*}\right)\left(Q_{n} T x_{k}\right)\right| \\
& \geq \epsilon-\frac{\epsilon \mathcal{M}\|T\|}{\epsilon 2 \mathcal{M}\|T\|}=\frac{\epsilon}{2}
\end{aligned}
$$

Escrevendo $f=Q_{n}^{*} y^{*}$, a equação anterior fica $\left|T^{*} f\left(x_{k}\right)\right| \geq \epsilon / 2$. Logo para todo $n$ suficientemente grande

$$
\begin{aligned}
\left\|P_{n}^{*}\left(T^{*} f\right)-T^{*} f\right\| & \geq\left|P_{n}^{*}\left(T^{*} f x_{n}\right)-T^{*} f x_{n}\right| \\
& =\left|T^{*} f\left(x_{n}\right)\right| \geq \epsilon / 2
\end{aligned}
$$

um absurdo, pois $T^{*} f \in X^{*}$ e $\left\{B_{n}\right\}_{n=1}^{\infty}$ é contrátil.

PASSO 2. Para cada $\epsilon>0$ e $n \in \mathbb{N}$ existe um inteiro $m \in \mathbb{N}$ tal que para todo $x \in P_{n} X$ temos $\left\|T x-Q_{m} T x\right\| \leq \epsilon\|x\|$

Observemos da compacidade de $\mathcal{S}_{P_{n} X}=\left\{x \in P_{n} X ;\|x\|=1\right\}$ que dado $\delta>0$ existem $x_{1}, \ldots, x_{n} \in \mathcal{S}_{P_{n} X}$ tais que para cada $x \in \mathcal{S}_{P_{n} X}$ e algum $i \in\{1, \ldots, n\},\left\|x-x_{i}\right\|<\delta$.

Sabemos que para cada $i, \lim _{k \rightarrow \infty} Q_{k} T x_{i}=T x_{i}$. Então existe $m \in \mathbb{N}$ tal que

$$
\left\|T x_{i}-Q_{k} T x_{i}\right\| \leq \epsilon
$$


para todo $k>m$. Assim, para $x \in \mathcal{S}_{P_{n} X}$

$$
\begin{aligned}
\left\|T x-Q_{m} T x\right\| & \leq\left\|T x-T x_{i}\right\|+\left\|T x_{i}-Q_{m} T x_{i}\right\|+\left\|Q_{m} T x_{i}-Q_{m} T x\right\| \\
& \leq\|T\| \delta+\epsilon / 2+\left\|Q_{m}\right\|\|T\| \delta \\
& =\epsilon / 2+(\|T\|+\mathcal{M}\|T\|) \delta
\end{aligned}
$$

Logo, tomando $\delta=\epsilon / 2(\|T\|+\mathcal{M}\|T\|)$, temos

$$
\left\|T x-Q_{m} T x\right\| \leq \epsilon\|x\|
$$

para todo $x \in P_{n} X$.

Usando as observações anteriores, vamos construir duas sequências de inteiros $1=m_{0}<$ $m_{1}<m_{2}<\ldots$ e $1=k_{0}<k_{1}<k_{2}<\ldots$ da seguinte maneira: Pelo PASSO 1. existe $m_{1}>1$ tal que se $P_{m_{1}} x=0$ então $\left\|Q_{k_{0}} T x\right\| \leq \epsilon_{2} / 2\|x\|$. Agora pelo PASSO 2. existe $k_{1}>1$ tal que se $x \in P_{m_{1}} X$, então $\left\|T x-Q_{k_{1}} T x\right\| \leq \epsilon_{1} / 2\|x\|$.

Continuando desta forma, para cada $j>1$ seja $m_{j}>m_{j-1}$ tal que $\left\|Q_{k_{j-1}} T x\right\| \leq \epsilon_{j+1} / 2\|x\|$ sempre que $P_{m_{j}} x=0$. Também, escolha $k_{j}>k_{j-1}$ tal que para cada $x \in P_{m_{j}} X$, tem-se $\left\|T x-Q_{k_{j}} T x\right\| \leq \epsilon_{j} / 2\|x\|$.

Assim para cada $x \in X_{m_{j-1}+1} \oplus \cdots \oplus X_{m_{j}}$

$$
\begin{aligned}
\left\|T x-\left(Q_{k_{j}} T x-Q_{k_{j-2}} T x\right)\right\| & \leq\left\|T x-Q_{k_{j}} T x\right\|+\left\|Q_{k_{j-2}} T x\right\| \\
& \leq \epsilon_{j} / 2\|x\|+\epsilon_{j} / 2\|x\| \\
& =\epsilon_{j}\|x\|
\end{aligned}
$$

observamos que $Q_{k_{j}} T x-Q_{k_{j-2}} T x \in Y_{k_{j-2}+1} \oplus \cdots \oplus Y_{k_{j-1}} \oplus \cdots \oplus Y_{k_{j}-1}$. Portanto as sequências $\left\{1, m_{1}+1, m_{2}+1, \ldots\right\}$ e $\left\{1, k_{1}+1, k_{2}+1, \ldots\right\}$ determinam os 'blockings' de $X$ e $Y$ respectivamente pedidos no teorema.

Notamos na demonstração do teorema anterior, que para cada $x \in B_{i}^{\prime}$ a existência de $y \in C_{i-1}^{\prime} \oplus C_{i}^{\prime}$ tal que $\|T x-y\| \leq \epsilon_{i}\|x\|$ depende linearmente de $x$. Logo o Teorema (1.51) pode ser enunciado como:

Teorema 1.52. Seja $T: X \rightarrow Y$ um operador linear continuo entre espaços de Banach $X$ e $Y$. Sejam $X=\sum X_{n}$ uma F.D.D contrátil de $X$ e $Y=\sum Y_{n}$ uma F.D.D de Y. Então para cada sequência $\left(\epsilon_{n}\right)_{n}$ com limite 0 , existem sequências estritamente crescentes $\left(m_{j}\right)_{j}$ e $\left(k_{j}\right)_{j}$ e aplicações lineares $T_{j}: X_{j}^{\prime} \rightarrow Y_{j-1}^{\prime} \oplus Y_{j}^{\prime}$ tais que $\left\|T x-T_{j} x\right\| \leq \epsilon_{j}\|x\|$ para todo $x \in X_{j}^{\prime}$, onde $X_{j}^{\prime}=X_{m_{j-1}+1} \oplus \cdots \oplus X_{m_{j}}$ e $Y_{j}^{\prime}=Y_{m_{j-1}+1} \oplus \cdots \oplus Y_{m_{j}}$. 
Lema 1.53. Sejam $X$ e $Y$ espaços de Banach e $X=\sum X_{n}$ uma decomposição de Schauder de $X$. Seja $\sum_{n=1}^{\infty} \epsilon_{n}=\epsilon$ uma serie convergente de números reais não negativos. Se existem aplicações lineares $T_{n}: X_{n} \rightarrow Y$ tais que $\left\|T_{n}\left(x_{n}\right)\right\| \leq \epsilon_{n}\left\|x_{n}\right\|$ para todo $x_{n} \in X_{n}$, então o operador $T(x):=\sum_{n=1}^{\infty} T_{n}\left(x_{n}\right)$, onde $x=\sum x_{n}$, está bem definido, é contínuo e $\|T\| \leq 2 \mathcal{K} \epsilon$ sendo $\mathcal{K}$ a constante da decomposição $\left\{X_{n}\right\}_{n=1}^{\infty}$.

Demonstração. Seja $x \in X$, vamos mostrar inicialmente que $\sum_{n} T_{n}\left(x_{n}\right)$ converge. Suponhamos que $\left\|x_{n}\right\| \leq 1$ para todo $n \in \mathbb{N}$. Assim

$$
\sum_{n=1}^{\infty}\left\|T_{n}\left(x_{n}\right)\right\| \leq \sum_{n=1}^{\infty} \epsilon_{n}=\epsilon
$$

logo como $Y$ é de Banach, obtemos que $\sum_{n=1}^{\infty} T_{n}\left(x_{n}\right)$ converge. Para o caso geral, observemos que

$$
\left\|x_{n}\right\|=\left\|\left(P_{n}-P_{n-1}\right)(x)\right\| \leq 2 \mathcal{K}\|x\|
$$

assim, aplicamos o resultado anterior a $x_{n} / 2 \mathcal{K}\|x\|$ e obtemos pela linearidade de $T_{n}$, que $\sum_{n} T_{n}\left(x_{n}\right)$ converge e

$$
\left\|\sum_{n=1}^{\infty} T_{n}\left(x_{n}\right)\right\| \leq 2 \mathcal{K} \epsilon\|x\|
$$

Agora é claro que $T(\lambda x)=\lambda T(x)$ e $T(x+y)=T(x)+T(y)$ para todo $\lambda \in \mathbb{K}$ e $x, y \in X$. Assim, $T: X \rightarrow Y$ é um operador linear contínuo tal que $\|T\| \leq 2 \mathcal{K} \epsilon$.

Juntando os dois resultados anteriores, obtemos

Teorema 1.54. Sejam $X$ e $Y$ espaços de Banach, $X=\sum X_{n}$ uma F.D.D contrátil de $X$ e $Y=\sum Y_{n}$ uma F.D.D de $Y$. Considere $\sum_{n=1}^{\infty} \epsilon_{n}=\epsilon$ uma serie convergente de números reais positivos. Então dado um operador continuo $T: X \rightarrow Y$, existem 'blockings' $\left\{X_{n}^{\prime}\right\}_{n=1}^{\infty}$ de $\left\{X_{n}\right\}_{n=1}^{\infty} e\left\{Y_{n}^{\prime}\right\}_{n=1}^{\infty}$ de $\left\{Y_{n}\right\}_{n=1}^{\infty}$ e um operador linear contínuo $\widetilde{T}: X \rightarrow Y$ tais que

(a) $\widetilde{T}(x) \in Y_{i-1}^{\prime} \oplus Y_{i}^{\prime}$ para todo $x \in X_{i}^{\prime}$

(b) $\|T(x)-\widetilde{T}(x)\| \leq \epsilon_{i}\|x\|$ para todo $x \in X_{i}^{\prime}$

(c) $\|\widetilde{T}\| \leq\|T\|+\epsilon$

Corolario 1.55. Sejam $X$ e $Y$ espaços de Banach, $X=\sum X_{n}$ uma F.D.D contrátil de $X$ e $Y=\sum Y_{n}$ uma F.D.D de $Y$. Considere $\sum_{n=1}^{\infty} \epsilon_{n}=\epsilon$ uma serie convergente de números reais positivos. Então dado um operador continuo $T: X \rightarrow Y$, existem 'blockings' $\left\{X_{n}^{\prime}\right\}_{n=1}^{\infty}$ de $\left\{X_{n}\right\}_{n=1}^{\infty}$ e $\left\{Y_{n}^{\prime \prime}\right\}_{n=1}^{\infty}$ de $\left\{Y_{n}\right\}_{n=1}^{\infty}$ e um operador continuo $\widetilde{T}: X \rightarrow Y$ tais que

(a) $\widetilde{T}(x) \in Y_{n}^{\prime \prime}$ para todo $x \in X_{2 n}^{\prime}$ 
(b) $\|T(x)-\widetilde{T}(x)\| \leq \epsilon_{n}\|x\|$ para todo $x \in X_{n}^{\prime}$

(c) $\|\widetilde{T}\| \leq\|T\|+\epsilon$

Demonstração. Basta tomar $Y_{n}^{\prime \prime}=Y_{2 n-1}^{\prime} \oplus Y_{2 n}^{\prime}$. Onde $\widetilde{T},\left\{X_{n}^{\prime}\right\}_{n=1}^{\infty}$ e $\left\{Y_{n}^{\prime}\right\}_{n=1}^{\infty}$ são obtidos pelo teorema anterior. 


\section{Capítulo 2}

\section{Somas torcidas}

Neste capítulo estudamos o conceito de soma torcida em $F$-espaços localmente limitados. Mostramos que toda soma torcida está associada a um operador quase-linear e estudamos simplificações na construção desse tipo de operadores.

Também abordamos o problema de somas torcidas de espaços de Banach, que por um exemplo exibido por Ribe [Rib79], nem sempre é um espaço de Banach. Para isto, será estudada a propriedade de B-convexidade e demonstraremos que toda soma torcida de espaços de Banach B-convexos é um espaço de Banach B-convexo.

\subsection{Somas torcidas}

Definição 2.1. Consideremos $X$ e $Y$ F-espaços. Uma soma torcida de $X$ e $Y$ é um F-espaço $Z$ que possui um subespaço $X^{\prime}$ isomorfo a $X$ tal que $Z / X^{\prime}$ seja isomorfo a $Y$.

A maioria dos teoremas deste capítulo serão estabelecidos para $F$-espaços localmente limitados. Esta classe de espaços é a mais geral e interessante para estudar somas torcidas, pois nos $F$-espaços é válido o teorema da aplicação aberta e, como será demonstrado na próxima secção, toda soma torcida de $F$-espaços localmente limitados é um $F$-espaço localmente limitado.

Usaremos a notação \|. || para referirmos à quase-norma que gera a topologia do $F$-espaço localmente limitado(Definição (1.37)).

Definição 2.2. Sejam $X, Y$ e $Z$ F-espaços. Vamos dizer que

$$
0 \rightarrow X \stackrel{j}{\rightarrow} Z \stackrel{q}{\rightarrow} Y \rightarrow 0
$$

é uma sequência exata curta de F-espaços se existem aplicações lineares contínuas $j: X \rightarrow Z$ e $q: Z \rightarrow Y$ tais que

- jé injetora.

- q é sobrejetora. 
- $j(X)=\operatorname{ker} q$.

Lema 2.3. Sejam $X, Y$ e $Z$ F-espaços. Então $Z$ é uma soma torcida de $X$ e $Y$ se, e somente se, existe uma sequência exata curta $0 \rightarrow X \stackrel{j}{\rightarrow} Z \stackrel{q}{\rightarrow} Y \rightarrow 0$.

Demonstração. Suponhamos $Z$ uma soma torcida de $X$ e $Y$. Se $X^{\prime}$ é um subespaço de $Z$ tal que existem $T: X \rightarrow X^{\prime}$ e $S: Z / X^{\prime} \rightarrow Y$ isomorfismos lineares, então

$$
0 \rightarrow X \stackrel{T}{\rightarrow} Z \stackrel{S \circ \pi}{\rightarrow} Y \rightarrow 0
$$

é uma sequência exta curta, onde $\pi: Z \rightarrow Z / X^{\prime}$ é a aplicação quociente.

Reciprocamente, se $0 \rightarrow X \stackrel{j}{\rightarrow} Z \stackrel{q}{\rightarrow} Y \rightarrow 0$ é uma sequência exata curta, temos que $j(X)$ é um subespaço fechado de $Z$, logo pelo teorema da aplicação aberta em $F$-espaços, $X$ é isomorfo a $j(X)$ e $Z / \operatorname{ker} q$ é isomorfo a $Y$. Portanto, escrevendo $X^{\prime}=j(X)$, obtemos que $X$ é isomorfo a $X^{\prime}$ e $Z / X^{\prime}$ é isomorfo a $Y$ e assim, $Z$ é soma torcida de $X$ e $Y$.

Definição 2.4. Sejam $X$ e $Y F$-espaços e $Z_{1}, Z_{2}$ duas sumas torcidas de $X$ e $Y$. Dizemos que $Z_{1}$ e $Z_{2}$ são equivalentes, se existe um operador linear contínuo $T: Z_{1} \rightarrow Z_{2}$ tal que o seguinte diagrama comuta

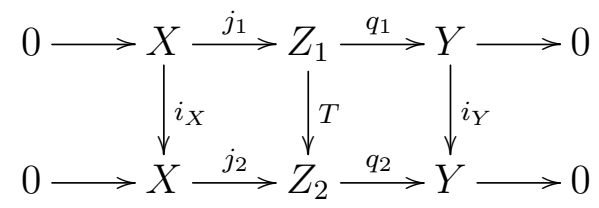

Observação 2.5. Observemos que na definição anterior $T$ deve ser um isomorfismo:

- $j_{2}(x)=T\left(j_{1} x\right), x \in X$

- $q_{2}(T z)=q_{1}(z), z \in Z_{1}$

- $T$ é injetor. Seja $z \in Z_{1}$ tal que $T z=0$, então $q_{1}(z)=q_{2}(z)=0$. Logo $z=j_{1}(x)$, $x \in X ; \operatorname{assim} j_{2}(x)=T\left(j_{1}(x)\right)=T(z)=0$ implica $x=0$ e portanto $z=0$.

- $T$ é sobrejetor. Seja $z_{2} \in Z_{2}$, como $q\left(z_{2}\right) \in Y$ e $q_{1}$ é sobrejetor, existe $z_{1} \in Z_{1}$ tal que $q_{1}\left(z_{1}\right)=q_{2}\left(z_{2}\right)$. Também $q_{1}\left(z_{1}\right)=q_{2}\left(T z_{1}\right)$, então $q_{2}\left(T z_{1}-z_{2}\right)=0$; isto é, existe $x \in X$ tal que $j_{2}(x)=T z_{1}-z_{2}$ como $j_{2}(x)=T\left(j_{1} x\right)$, concluímos $T\left(z_{1}-j_{1} x\right)=T z_{1}-T\left(j_{1} x\right)=$ $z_{2}$.

- Pelo teorema da aplicação aberta em F-espaços (Corolario (1.34)) T é um isomorfismo.

Nesta secção vamos estudar varias propriedades das somas torcidas, em particular mostraremos que geram e são geradas por aplicações quase-lineares.

Definição 2.6. Uma função $F: Y \rightarrow X$ chama-se quase-linear se satisfaz:

(a) $F(\lambda y)=\lambda F(y)$, para todo $y \in Y$ e $\lambda \in K$ 
(b) $\left\|F\left(y_{1}+y_{2}\right)-F\left(y_{1}\right)-F\left(y_{2}\right)\right\| \leq M\left(\left\|y_{1}\right\|+\left\|y_{2}\right\|\right)$, para todo $y_{1}, y_{2} \in Y$ onde $M$ é uma constante independente de $y_{1}, y_{2}$.

Consideremos $X$ e $Y$ F-espaços quase-normados e $F: Y \rightarrow X$ uma aplicação quaselinear. Observemos que a fórmula

$$
\|(x, y)\|_{F}=\|y\|+\|x-F(y)\|
$$

define uma quase-norma no espaço vetorial $X \times Y$ (onde usamos o mesmo símbolo para as diferentes quase-normas).

Com efeito, as propriedades (Q1), (Q2) e (Q3) são claras. Provemos (Q4)

$$
\begin{aligned}
\left\|\left(x_{1}+x_{2}, y_{1}+y_{2}\right)\right\|_{F} & =\left\|y_{1}+y_{2}\right\|+\left\|\left(x_{1}+x_{2}\right)-F\left(y_{1}+y_{2}\right)\right\| \\
& \leq k\left(\left\|y_{1}\right\|+\left\|y_{2}\right\|\right)+k\left\|\left(x_{1}-F\left(y_{1}\right)\right)+\left(x_{2}-F\left(y_{2}\right)\right)\right\| \\
& +k\left\|F\left(y_{1}+y_{2}\right)-F\left(y_{1}\right)-F\left(y_{2}\right)\right\| \\
& \leq k^{2}\left(\left\|y_{1}\right\|+\left\|y_{2}\right\|+\left\|\left(x_{1}-F\left(y_{1}\right)\right)\right\|+\left\|\left(x_{2}-F\left(y_{2}\right)\right)\right\|\right) \\
& +k M\left(\left\|y_{1}\right\|+\left\|y_{2}\right\|\right) \\
& \leq k(k+M)\left(\left\|y_{1}\right\|+\left\|\left(x_{1}-F\left(y_{1}\right)\right)\right\|+\left\|y_{2}\right\|+\left\|\left(x_{2}-F\left(y_{2}\right)\right)\right\|\right) \\
& =k(k+M)\left(\left\|\left(x_{1}, y_{1}\right)\right\|_{F}+\left\|\left(x_{2}, y_{2}\right)\right\|_{F}\right)
\end{aligned}
$$

onde $k$ é um módulo de concavidade para as quase-normas em $X$ e $Y$.

Agora mostraremos que o subespaço $X^{\prime}=\{(x, 0): x \in X\}$ de $X \oplus_{F} Y$ é isométrico a $X$ e que $\left(X \oplus_{F} Y\right) / X^{\prime}$ é isométrico a $Y$. Com efeito, definamos $j(x)=(x, 0)$, assim

$$
\|j(x)\|_{F}=\|(x, 0)\|_{F}=\|x\|
$$

Completamos a afirmação observando

$$
\begin{aligned}
\left\|\left(x_{0}, y\right)+X^{\prime}\right\|_{F} & =\inf \left\{\left\|\left(x_{0}, y\right)+(x, 0)\right\|_{F}: x \in X\right\} \\
& =\inf \{\|x-F(y)\|+\|y\|: x \in X\} \\
& =\|y\|
\end{aligned}
$$

Portanto $\left(X \times Y,\|\cdot\|_{F}\right)$ é uma soma torcida de $X$ e $Y$.

Definição 2.7. Se $X$ e $Y$ são F-espaços quase-normados e $F: Y \rightarrow X$ é uma aplicação quase-linear, denotaremos por $X \oplus_{F} Y$ a soma torcida $\left(X \times Y,\|\cdot\|_{F}\right)$ obtida no comentário anterior.

Observemos que todas as somas torcidas são da forma anterior:

Teorema 2.8. Toda soma torcida $Z$ de $X$ e $Y$ é equivalente a $X \oplus_{F} Y$ para alguma aplicação quase-linear $F: Y \rightarrow X$. 
Demonstração. Considere $K$ um módulo de concavidade para as quase-normas de $X, Y$ e $Z$. Suponhamos que $0 \rightarrow X \stackrel{j}{\rightarrow} Z \stackrel{q}{\rightarrow} Y \rightarrow 0$ é uma sequência exata curta que define a soma torcida. Pelo Lema (1.6), existe uma aplicação linear $\theta: Y \rightarrow Z$ tal que $q \theta=i d_{Y}$. Dado que $q: Z \rightarrow Y$ é aberta, então existe uma constante $C$ e uma aplicação $\phi: Y \rightarrow Z$ tal que

(a) $\|\phi(y)\| \leq C\|y\|$, para todo $y \in Y$.

(b) $q \phi(y)=y$, para todo $y \in Y$.

(c) $\phi(\lambda y)=\lambda \phi(y)$, para todo $y \in Y$ e $\lambda \in \mathbb{K}$.

Com efeito, pelo teorema da aplicação aberta existe uma constante $C>0$ tal que $\frac{1}{C} \mathfrak{B}_{Y} \subseteq$ $q\left(\mathfrak{B}_{Z}\right)$. Vamos definir $\phi$ inicialmente em $S_{Y}=\{y \in Y:\|y\|=1\}$. Considere sobre $S_{Y}$ a seguinte relação de equivalência, $y_{1} R y_{2}$ se, e somente se, existe $\lambda \in \mathbb{K}$ com $|\lambda|=1$ tal que $y_{2}=\lambda y_{1}$. Para cada $y \in S_{Y}$, denotemos por $\bar{y}$ a classe de equivalência de $y$. Pelo axioma de escolha, consideremos $J=\bigcup_{\alpha \in I}\left\{y_{\alpha}\right\}$ um conjunto que contém um único representante de cada classe de equivalência. Para cada $y_{\alpha} \in J$, definamos $\phi\left(y_{\alpha}\right)=C z_{\alpha}$ onde $y_{\alpha}=q\left(C z_{\alpha}\right)$ para algum $z_{\alpha} \in \mathfrak{B}_{Z}$. Deste modo $\left\|\phi\left(y_{\alpha}\right)\right\| \leq C$ para todo $\alpha \in I$. Agora dado $y \in S_{Y}$, definimos $\phi(y)=\phi\left(y_{\alpha}\right)$ se $y \in \overline{y_{\alpha}}$. E para cada $\lambda \in \mathbb{K}$ e $y \in S_{Y}$ definamos $\phi(\lambda y)=\lambda \phi(y)$. Assim, para $y \in Y, y \neq 0$ arbitrário

$$
\phi(y)=\|y\| \phi\left(\frac{y}{\|y\|}\right)
$$

e com isto $\phi$ satisfaz as propriedades enunciadas.

Como $\phi(y)-\theta(y) \in \operatorname{ker}(q)=j(X)$ e $j$ é injetora, então definamos $F(y)=j^{-1}(\phi(y)-\theta(y))$. Seja $L$ tal que $\left\|j^{-1} z\right\| \leq L\|z\|$ para todo $z \in j(X)$. Assim, para todo $y_{1}, y_{2} \in Y$

$$
\begin{aligned}
\left\|F\left(y_{1}+y_{2}\right)-F\left(y_{1}\right)-F\left(y_{2}\right)\right\| & =\left\|j^{-1}\left(\phi\left(y_{1}+y_{2}\right)-\phi\left(y_{1}\right)-\phi\left(y_{2}\right)\right)\right\| \\
& \leq L\left\|\phi\left(y_{1}+y_{2}\right)-\phi\left(y_{1}\right)-\phi\left(y_{2}\right)\right\| \\
& \leq L K^{2}\left(\left\|\phi\left(y_{1}+y_{2}\right)\right\|+\left\|\phi\left(y_{1}\right)\right\|+\left\|\phi\left(y_{2}\right)\right\|\right) \\
& \leq L K^{2} C\left(\left\|y_{1}+y_{2}\right\|+\left\|y_{1}\right\|+\left\|y_{2}\right\|\right) \\
& \leq 2 L K^{3} C\left(\left\|y_{1}\right\|+\left\|y_{2}\right\|\right) .
\end{aligned}
$$

Portanto, $F$ é um operador quase-linear, pois é claro que $F(\lambda y)=\lambda F(y)$ para todo $\lambda \in \mathbb{K}$. Agora definamos $T: Z \rightarrow X \oplus_{F} Y$ mediante a fórmula

$$
T(z)=\left(j^{-1}(z-\theta q z), q z\right) .
$$


Então

$$
\begin{aligned}
\|T(z)\|_{F} & =\|q z\|+\left\|j^{-1}(z-\theta(q z))-j^{-1}(\phi(q z))-\theta(q z)\right\| \\
& =\|q z\|+\left\|j^{-1}(z-\phi(q z))\right\| \\
& \leq\|q z\|+L\|z-\phi(q z)\| \\
& \leq\|q z\|+K L(\|z\|+\|\phi(q z)\|) \\
& \leq\|q\|\|z\|+K L(\|z\|+C\|q\|\|z\|) \\
& =(\|q\|+K L+K L C\|q\|)\|z\|
\end{aligned}
$$

Com isto, $T$ é contínuo. Observemos que o diagrama

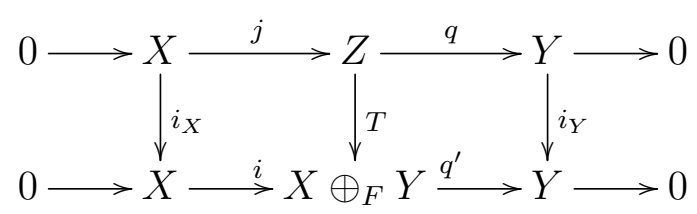

comuta, pois

- $T(j x)=\left(j^{-1}(j x-\theta q(j x)), q(j x)\right)=(x, 0)=i(x)$ para todo $x \in X$

- $q^{\prime} T(z)=q z$ para todo $z \in Z$.

O que completa a prova do teorema.

Definição 2.9. As aplicações quase-lineares $F, G: Y \rightarrow X$ são equivalentes se $X \oplus_{F} Y$ e $X \oplus_{G} Y$ são somas torcidas equivalentes.

Teorema 2.10. As aplicações quase-lineares $F$ e $G$ são equivalentes se, e somente, se existe uma constante $M$ e uma aplicação linear $A: Y \rightarrow X$ tal que

$$
\|F(y)-G(y)+A(y)\| \leq M\|y\|, \quad y \in Y
$$

Demonstração. Suponhamos que $F$ e $G$ são equivalentes e seja $T: X \oplus_{F} Y \rightarrow X \oplus_{G} Y$ um operador linear contínuo tal que o diagrama

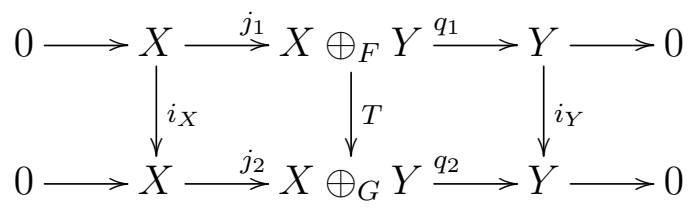

seja comutativo. Assim, $T\left(j_{1}(x)\right)=j_{2}(x)$ implica que $T(x, 0)=(x, 0)$ e de $q_{1}(x, y)=$ $q_{2}(T(x, y))$ temos $y=q_{2}(T(0, y))$. Escrevamos $\pi_{X}: X \oplus_{F} Y \rightarrow X$ a projeção sobre $X\left(\pi_{X}(x, y)=x\right)$ e definamos a aplicação $A(y)=\pi_{X} T(0, y), y \in Y$. Claramente $A$ é linear e 


$$
T(x, y)=T(x, 0)+T(0, y)=(x, 0)+(A(y), y)=(x+A(y), y)
$$

Então $T(F(y), y)=(F(y)+A(y), y)$ e

$$
\begin{aligned}
\|T(F(y), y)\|_{G} & =\|y\|+\|F(y)+A(y)-G(y)\| \\
\|(F(y), y)\|_{F} & =\|y\|+\|F(y)-F(y)\|=\|y\|
\end{aligned}
$$

Logo, pela continuidade de $T$

$$
\|F(y)-G(y)+A(y)\| \leq\|T\|\|y\|, \quad y \in Y
$$

Reciprocamente, se $F, G$ e $A$ satisfazem as hipóteses, defina $T: X \oplus_{F} Y \rightarrow X \oplus_{G} Y$ como sendo $T(x, y)=(x+A(y), y)$. É claro que $T$ é linear e contínuo, pois

$$
\begin{aligned}
\| T(x), y) \|_{G} & =\|y\|+\|x+A(y)-G(y)\| \\
& \leq k(\|y\|+\|x-F(y)\|+\|F(y)-G(y)+A(y)\|) \\
& \leq k(m+1)(\|y\|+\|x-F(y)\|) \\
& =k(m+1)\|(x, y)\|_{F}
\end{aligned}
$$

Pela construção, $T$ faz que o diagrama comute, com isto a prova fica completa.

\subsection{Propriedades preservadas pelas somas torcidas}

Seja $P$ uma propriedade definida para espaços quase-normados. Dizemos que $P$ é uma propriedade dos três espaços na categoria dos espaços quase-normados se todo espaço quasenormado $X$ possui a propriedade $P$, sempre que exista um subespaço fechado $M$ de $X$ tal que os dois, $M$ e $X / M$, satisfazem a propriedade $P$. No caso que uma propriedade $P$, dos três espaços, seja invariante por isomorfismos(i.é. se $X$ e $X^{\prime}$ são espaços isomorfos tal que se $X$ satisfaz $P$ então $X^{\prime}$ também deve satisfazer $P$ ) temos que $P$ é conservada por somas torcidas, ou seja, toda soma torcida de espaços com a propriedade $P$ também satisfaz a propriedade $P$.

Nesta seção vamos dar exemplos de algumas propriedades elementares que são dos três espaços (usamos como referência [CG97]). Em particular, pelos Teoremas (2.11) e (2.14) fica claro que toda soma torcida de $F$-espaços localmente limitados é um $F$-espaço localmente limitado.

Ribe(ver [Rib79]) apresentou um exemplo de uma soma torcida de espaços de Banach que não é localmente convexa, e portanto, não é um espaço de Banach. Assim a propriedade de ser Banach não é dos três espaços. Este caso será analizado na proxima seção. 
Teorema 2.11. Ser completo é uma propriedade dos três espaços.

Demonstração. Sejam $X$ um espaço quase-normado e $M$ um subespaço fechado de $X$ tal que $M$ e $X / M$ sejam completos. Consideremos $\left(x_{n}\right)_{n}$ uma sequência de Cauchy em $X$. Assim $\left(x_{n}+M\right)_{n}$ é uma sequência de Cauchy em $X / M$ pois $\left\|\left(x_{n}-x_{m}\right)+M\right\| \leq\left\|x_{n}-x_{m}\right\|$. Com isto, existe $y \in X$ tal que $\lim x_{n}+M=y+M$. Pelas propriedades da norma do quociente, para cada $n \in \mathbb{N}$ existe $y_{n} \in X$ com $y_{n}+M=\left(x_{n}-y\right)+M$ tal que

$$
\left\|y_{n}\right\| \leq\left\|\left(x_{n}-y\right)+M\right\|+2^{-n}
$$

em consequência $\lim y_{n}=0$. Notemos que $x_{n}-y-y_{n}$ é uma sequência de Cauchy em $M$, que deve convergir para algum $z \in M$. Concluímos que $x_{n}=\left(x_{n}-y-y_{n}\right)+\left(y+y_{n}\right)$ converge para $z+y \in X$. Logo $X$ é completo.

Teorema 2.12. A propriedade de ter dimensão finita é dos três espaços.

Demonstração. Sejam $X$ um espaço quase-normado e $M$ um subespaço de $X$ tal que $M$ e $X / M$ possuem dimensão finita. Então se $\left\{m_{1}, \ldots, m_{n}\right\}$ é uma base para $M$ e $\left\{x_{1}+M, \ldots, x_{k}+M\right\}$ é uma base de $X / M$, é imediato obsevar que $\left\{m_{1}, \ldots, m_{n}, x_{1} \ldots, x_{k}\right\}$ é uma base para $X$.

Teorema 2.13. Ser separável é uma propriedade dos três espaços.

Demonstração. Consideremos $X$ um espaço quase-normado e $M$ um subespaço de $X$ tal que $M$ e $X / M$ sejam separáveis. Suponhamos que $\left\{m_{1}, m_{2}, \ldots\right\}$ e $\left\{x_{1}+M, x_{2}+M, \ldots\right\}$ sejam conjuntos densos enumeráveis em $M$ e $X / M$, respetivamente. Vamos mostrar que a coleção $\left\{x_{i}+m_{j}\right\}_{i, j \in \mathbb{N}}$ é densa em $X$.

Sejam $x \in X$ e $\epsilon>0$. Então existe $x_{i}+M$ tal que

$$
\left\|\left(x-x_{i}\right)+M\right\|<\epsilon / 3 K
$$

onde $K$ é o módulo de concavidade da quase-norma. Tomemos $y \in X \operatorname{com} y+M=$ $\left(x-x_{i}\right)+M$ tal que

$$
\|y\|<\left\|\left(x-x_{i}\right)+M\right\|+\epsilon / 3 K .
$$

Assim, como $x-x_{i}-y \in M$, existe $m_{j} \in M$ tal que $\left\|x-x_{i}-y-m_{j}\right\|<\epsilon / 3 K$. Portanto

$$
\left\|x-\left(x_{i}+m_{j}\right)\right\| \leq K\left(\left\|x-x_{i}-y-m_{j}\right\|+\|y\|\right)<\epsilon
$$

o que completa a prova.

Teorema 2.14. Ser localmente limitado é uma propriedade dos três espaços.

Demonstração. Sejam $X$ um $F$-espaço e $M$ um subespaço de $X$ tal que $M$ e $X / M$ sejam espaços localmente limitados. Tomemos $U$ uma vizinhança de 0 em $X$, tal que $U \cap M$ e $\pi(U)$ sejam limitados, onde $\pi: X \rightarrow X / M$ é a aplicação quociente. Pelo Lema (1.28) existe 
uma vizinhança equilibrada $V$ de 0 tal que $V+V \subseteq U$. Vamos mostrar que $V$ é limitada. Por absurdo, se não fosse, existiriam uma vizinhança equilibrada $W$ de 0 e uma sequência $\left(x_{n}\right)_{n}$ de elementos em $V$ tais que $x_{n} \notin n^{2} W$. Assim a sequência $\frac{1}{n} \pi\left(x_{n}\right)$ converge para 0 , pois $\pi(V)$ é limitada. Também para cada $n \in \mathbb{N}$ existe $m_{n} \in M$ tal que $\lim \frac{1}{n} x_{n}+m_{n}=0$. Logo, existe $N \in \mathbb{N}$ tal que $\frac{1}{n} x_{n}+m_{n} \in V$ para todo $n \geq N$. Como $V$ é equilibrada, temos

$$
m_{n}=\left(\frac{1}{n} x_{n}+m_{n}\right)+\left(-\frac{1}{n} x_{n}\right) \in V+V \subseteq U .
$$

Com isto, a sequência $\left(m_{n}\right)$ é limitada, o que implica que $\left(\frac{1}{n} x_{n}\right)$ é limitada, pois soma de conjuntos limitados é limitado. Portanto, existe $\alpha>0$ tal que $x_{n} \in n \alpha V$ para todo $n \in \mathbb{N}$, em particular tomando $n \geq \alpha$, temos $x_{n} \in n^{2} V$ o que é uma contradição. Concluímos que $V$ é limitada.

Teorema 2.15. Reflexividade é uma propriedade dos três espaços.

Demonstração. Sejam $X$ um espaço normado e $M$ um subespaço fechado de $X$ tal que $M$ e $X / M$ sejam reflexivos. Para demonstrar o teorema é suficiente tomar $x^{* *} \in X^{* *}$ e mostrar que $x^{* *} \in Q(X)$, onde $Q$ é a aplicação canônica de $X$ em $X^{* *}$. Consideremos $T$ a aplicação isométrica entre os espaços $M^{\perp} \mathrm{e}(X / M)^{*}$ como no Lema (1.17)-(b), então $x^{* *} T^{-1} \in(X / M)^{* *}$ e pela reflexividade, existe $x \in X$ tal que para cada $m^{\perp} \in M^{\perp}$

$$
\begin{aligned}
x^{* *}\left(m^{\perp}\right)=\left(x^{* *} T^{-1}\right)\left(T m^{\perp}\right) & =T m^{\perp}(x+M) \\
& =m^{\perp}(x) \\
& =Q(x)\left(m^{\perp}\right)
\end{aligned}
$$

Assim $x^{* *}-Q(x) \in M^{\perp \perp}$ e pelo Lema (1.18) existe $m \in M$ tal que $x^{* *}-Q(x)=Q(m)$. Em consequência $x^{* *}=Q(x+m)$. Isto completa a prova.

\subsection{Somas torcidas de espaços de Banach}

O objetivo desta seção será estudar o conceito de B-convexidade e demonstrar que toda soma torcida de espaços de Banach B-convexos é um espaço de Banach também B-convexo.

Definição 2.16 (Funções de Rademacher). As funções de Rademacher são aplicações $\gamma_{n}$ : $[0,1] \rightarrow \mathbb{R}, n \in \mathbb{N}$, definidas por

$$
\gamma_{n}(t)=\operatorname{Sinal}\left(\sin 2^{n} \pi t\right)
$$

A $n$-ésima função de Rademacher toma valor constante +1 ou -1 , de forma alternada, nos intervalos do tipo $\left(k 2^{-n},(k+1) 2^{-n}\right)$ para $k=0, \ldots, 2^{n}-1$, começando com $\gamma_{n}(t)=+1$ para $t \in\left[0,2^{-n}\right]$. 
As seguintes fórmulas serão usadas com frequência em próximos argumentos.

Lema 2.17. Para cada sequência de sinais $\left(\epsilon_{i}\right)_{i=1}^{n}\left(\epsilon_{i}= \pm 1\right)$ existe um único $k \in\left\{0,1, \ldots, 2^{n}-1\right\}$, tal que

$$
\epsilon_{i}=\gamma_{i}(t), \quad t \in\left(\frac{k}{2^{n}}, \frac{k+1}{2^{n}}\right) \quad \text { para } \quad i=1, \ldots, n .
$$

Demonstração. Vamos provar este lema por indução sobre $n$. É claro que a afirmação é válida quando $n=1$. Supondo que também é válida para $n$, consideremos $\left(\epsilon_{i}\right)_{i=1}^{n+1}$ uma sequência de $n+1$ sinais. Pela hipótese de indução, seja $k_{n} \in\left\{0,1, \ldots, 2^{n}-1\right\}$ único tal que

$$
\epsilon_{i}=\gamma_{i}(t), \quad t \in\left(\frac{k_{n}}{2^{n}}, \frac{k_{n}+1}{2^{n}}\right) \quad \text { para } i=1, \ldots, n
$$

Agora $\gamma_{n+1}$ alterna de sinal só uma vez no intervalo $\left(\frac{k_{n}}{2^{n}}, \frac{k_{n}+1}{2^{n}}\right)$. Então $\epsilon_{n+1}=\gamma_{n+1}(t)$ quando $t \in\left(\frac{k}{2^{n+1}}, \frac{k+1}{2^{n+1}}\right)$ onde $k=2 k_{n}$ ou $k=2 k_{n}+1$ (só um dos dois). Portanto

$$
\epsilon_{i}=\gamma_{i}(t), \quad t \in\left(\frac{k}{2^{n+1}}, \frac{k+1}{2^{n+1}}\right) \quad \text { para } i=1, \ldots, n+1,
$$

já que $\left(\frac{k}{2^{n+1}}, \frac{k+1}{2^{n+1}}\right) \subseteq\left(\frac{k_{n}}{2^{n}}, \frac{k_{n}+1}{2^{n}}\right)$. O que completa o passo indutivo.

Lema 2.18. Seja $(X,\|\|$.$) um espaço quase-normado. Consideremos x_{1}, \ldots, x_{n}, \ldots, x_{n m} n m$ vetores em $X$. Então temos:

(a) $\int_{0}^{1}\left\|\sum_{k=1}^{n} \gamma_{k}(t) x_{k}\right\|^{2} d t=\frac{1}{2^{n}} \sum_{\epsilon_{k}= \pm 1}\left\|\sum_{k=1}^{n} \epsilon_{k} x_{k}\right\|^{2}$

(b) Para cada $s \in[0,1]$

$$
\int_{0}^{1}\left\|\sum_{k=1}^{n} \gamma_{k}(s)\left(\sum_{m(k-1)<j \leq m k} \gamma_{j}(t) x_{j}\right)\right\|^{2} d t=\int_{0}^{1}\left\|\sum_{j=1}^{m n} \gamma_{j}(u) x_{j}\right\|^{2} d u
$$

Onde a soma no lado direito de (a) é tomada sobre todas as escolhas de $n$ sinais $\epsilon=\left(\epsilon_{i}\right)_{i=1}^{n}$, ou seja, $\epsilon_{i}$ é +1 ou -1 para cada $i=1, \ldots, n$.

Demonstração. (a) Pelo lema anterior

$$
\begin{aligned}
\int_{0}^{1}\left\|\sum_{k=1}^{n} \gamma_{k}(t) x_{k}\right\|^{2} d t & =\sum_{j=0}^{2^{n}-1} \int_{\frac{j}{2^{n}}}^{\frac{j+1}{2^{n}}}\left\|\sum_{k=1}^{n} \gamma_{k}(t) x_{k}\right\|^{2} d t \\
& =\frac{1}{2^{n}} \sum_{\epsilon_{i}= \pm 1}\left\|\sum_{k=1}^{n} \epsilon_{k} x_{k}\right\|^{2}
\end{aligned}
$$

(b) Usando a parte (a) 


$$
\begin{aligned}
\int_{0}^{1}\left\|\sum_{k=1}^{n} \gamma_{k}(s)\left(\sum_{m(k-1)<j \leq m k} \gamma_{j}(t) x_{j}\right)\right\|^{2} d t & =\int_{0}^{1}\left\|\sum_{j=1}^{m n} \gamma_{j}(t)\left(\gamma_{k_{j}}(s) x_{j}\right)\right\|^{2} d t \\
& =\frac{1}{2^{n m}} \sum_{\epsilon_{j}= \pm 1}\left\|\sum_{j=1}^{n m} \epsilon_{j} \gamma_{k_{j}}(s) x_{j}\right\|^{2} \\
& =\frac{1}{2^{n m}} \sum_{\epsilon_{j}= \pm 1}\left\|\sum_{j=1}^{n m} \epsilon_{j} x_{j}\right\|^{2}
\end{aligned}
$$

onde $k_{j}$ é o inteiro $k$ tal que $j \in(m(k-1), m k]$. A última igualdade segue do fato que $\gamma_{k_{j}}(s)= \pm 1$ e a soma é tomada sobre todas as sequências de $n m$ sinais, então o produto $\epsilon_{j} \gamma_{k_{j}}(s)$ também percorre todas $2^{n m}$ possíveis escolhas de $n m$ sinais. Usando novamente (a) obtemos (b).

Definição 2.19. Seja $(X,\|\|$.$) um espaço quase-normado. Definamos$

$$
\begin{aligned}
a_{n}(X) & =\sup _{x_{1}, \ldots, x_{n} \in \mathfrak{B}_{X}}\left\|x_{1}+\cdots+x_{n}\right\| \\
b_{n}(X) & =\sup _{x_{1}, \ldots, x_{n} \in \mathfrak{B}_{X}} \min _{\epsilon_{i}= \pm 1}\left\|\sum_{i=1}^{n} \epsilon_{i} x_{i}\right\| \\
c_{n}^{2}(X) & =\sup _{\sum_{i=1}^{n}\left\|x_{i}\right\|^{2}=1} \frac{1}{n}\left(\frac{1}{2^{n}} \sum_{\epsilon_{i}= \pm 1}\left\|\sum_{i=1}^{n} \epsilon_{i} x_{i}\right\|^{2}\right)
\end{aligned}
$$

Quando seja clara a referência do espaço, escreveremos simplesmente $a_{n}, b_{n}, c_{n}^{2}$. Da definição, notamos que as sequências $a_{n}$ e $b_{n}$ são crescentes.

A partir do Lema (2.18) e usando a notação das funções de Rademacher, obtemos que $c_{n}^{2}(X)$ é a menor constante $c$ tal que

$$
\int_{0}^{1}\left\|\sum_{k=1}^{n} \gamma_{k}(t) x_{k}\right\|^{2} d t \leq c^{2} n\left(\sum_{k=1}^{n}\left\|x_{k}\right\|^{2}\right)
$$

para qualquer escolha $x_{1}, \ldots, x_{n} \in X$.

Lema 2.20. Seja $X$ um espaço quase-normado. As sequências $\left(a_{n}\right),\left(b_{n}\right)$ e $\left(c_{n}\right)$ são submultiplicativas no sentido

(a) $a_{n m} \leq a_{n} a_{m}$

(b) $b_{n m} \leq b_{n} b_{m}$

(c) $c_{n m} \leq c_{n} c_{m}$ 
para todo $n, m \in \mathbb{N}$.

Demonstração. (a). Sejam $x_{i j} \in X$ com $\left\|x_{i j}\right\| \leq 1$ para $1 \leq i \leq n$ e $1 \leq j \leq m$. Pela definição

$$
\left\|x_{i 1}+\cdots+x_{i m}\right\| \leq a_{m} \quad(i=1, \ldots, n)
$$

assim

$$
\frac{1}{a_{m}}\left\|\left(x_{11}+\cdots+x_{1 m}\right)+\cdots+\left(x_{n 1}+\cdots+x_{n m}\right)\right\| \leq a_{n}
$$

$\operatorname{logo} a_{n m} \leq a_{n} a_{m}$.

(b). Considere $x_{i j} \in X$ como en (a). Para cada $i$ escolha sinais $\theta_{i j}= \pm 1(j=1, \ldots, m)$ tais que

$$
\left\|\sum_{j=1}^{m} \theta_{i j} x_{i j}\right\| \leq b_{m} \quad(i=1, \ldots, n) .
$$

Escolha sinais $\eta_{i}= \pm 1, i=1, \ldots, n$ tais que

$$
\left\|\sum_{i=1}^{n} \eta_{i}\left(\frac{1}{b_{m}} \sum_{j=1}^{m} \theta_{i j} x_{i j}\right)\right\| \leq b_{n} .
$$

Logo se escrevemos $\epsilon_{i j}:=\eta_{i} \theta_{i j}$, obtemos

$$
\left\|\sum_{i, j} \epsilon_{i j} x_{i j}\right\| \leq b_{n} b_{m}
$$

Portanto $b_{n m} \leq b_{n} b_{m}$.

(c) Sejam $x_{1}, \ldots, x_{n m} n m$ vetores em $X$. Para cada $1 \leq k \leq n$ e $t \in[0,1]$ definamos

$$
y_{k}(t)=\sum_{m(k-1)<j \leq m k} \gamma_{j}(t) x_{j}
$$

Denotemos por $I=\int_{0}^{1} \int_{0}^{1}\left\|\sum_{k=1}^{n} \gamma_{k}(s) y_{k}(t)\right\|^{2} d s d t$. Segue da definição de $c_{n}$ que

$$
I \leq n c_{n}^{2} \sum_{k=1}^{n} \int_{0}^{1}\left\|y_{k}(t)\right\|^{2} d t \leq n m c_{n}^{2} c_{m}^{2} \sum_{j=1}^{n m}\left\|x_{j}\right\|^{2} .
$$

Por outro lado, o Lema (2.18)-(b) estabelece para cada $s \in[0,1]$

$$
\int_{0}^{1}\left\|\sum_{k=1}^{n} \gamma_{k}(s) y_{k}(t)\right\|^{2} d t=\int_{0}^{1}\left\|\sum_{j=1}^{m n} \gamma_{j}(u) x_{j}\right\|^{2} d u .
$$

Portanto

$$
\int_{0}^{1}\left\|\sum_{j=1}^{m n} \gamma_{j}(u) x_{j}\right\|^{2} d u=I \leq(m n)\left(c_{n} c_{m}\right)^{2} \sum_{j=1}^{n m}\left\|x_{j}\right\|^{2}
$$


e em consequência $c_{n m} \leq c_{n} c_{m}$.

Definição 2.21. Um espaço quase-normado é chamado B-convexo se $\lim _{n \rightarrow \infty} n^{-1} b_{n}=0$.

Pela propriedade sub-multiplicativa dos $b_{n}$, podemos notar que uma condição necessária e suficiente para que um espaço $X$ seja B-convexo é que exista um $m \in \mathbb{N}$ tal que $m^{-1} b_{m}<1$. Com efeito, esta condição é necessária. Para ver que é suficiente, seja $n \in \mathbb{N}$ arbitrário e $k \in \mathbb{N}$ tal que $m^{k} \leq n<m^{k+1}$. Assim, usando o Lema (2.20)

$$
n^{-1} b_{n} \leq m^{-k} b_{m^{k+1}} \leq m^{-k} b_{m^{k}} b_{m} \leq\left(m^{-1} b_{m}\right)^{k} b_{m}
$$

e portanto $\lim _{n \rightarrow \infty} n^{-1} b_{n}=0$.

Logo ser B-convexo significa que existem $n \in \mathbb{N}, n>1$ e um $\delta>0$ tal que para cada escolha de $n$-vetores $x_{1}, \ldots, x_{n} \in \mathfrak{B}_{X}$ existe uma escolha de sinais $\epsilon=\left(\epsilon_{i}\right)_{i=1}^{n}$ de tal forma que

$$
\left\|\epsilon_{1} x_{1}+\cdots+\epsilon_{n} x_{n}\right\| \leq(1-\delta) n
$$

Num espaço normado, isto pode-se entender como a propriedade que permite fazer múltiples cancelamentos entre quaisquer $n$ vetores de $\mathfrak{B}_{X}$ usando sinais $\epsilon_{i}= \pm 1$, isto é, a expressão $\epsilon_{1} x_{1}+\cdots+\epsilon_{n} x_{n}$ tem vários cancelamentos de modo que não se atinge a norma máxima.

Considere $x_{1}, \ldots, x_{n} \in \mathfrak{B}_{X}$. Segue da definição de $c_{n}$ que

$$
\frac{1}{2^{n}} \sum_{\epsilon_{i}= \pm 1}\left\|\sum_{i=1}^{n} \epsilon_{i} x_{i}\right\|^{2} \leq n c_{n}^{2}\left(\sum_{i=1}^{n}\left\|x_{i}\right\|^{2}\right) \leq n^{2} c_{n}^{2}
$$

Assim, existe uma escolha de sinais $\widetilde{\epsilon}=\left(\widetilde{\epsilon}_{k}\right)_{k=1}^{n}$ tal que

$$
\left\|\sum_{k=1}^{n} \widetilde{\epsilon}_{k} x_{k}\right\|^{2} \leq n^{2} c_{n}^{2}
$$

Portanto

$$
\min _{\epsilon_{i}= \pm 1}\left\|\sum_{i=1}^{n} \epsilon_{i} x_{i}\right\| \leq n c_{n}
$$

de onde concluímos que $n^{-1} b_{n} \leq c_{n}$. Suponhamos agora que $X$ é um espaço normado. A desigualdade

$$
\left\|\sum_{i=1}^{n} \epsilon_{i} x_{i}\right\|^{2} \leq\left(\left\|\sum_{i=1}^{n} x_{i}\right\|\right)^{2} \leq n \sum_{i=1}^{n}\left\|x_{i}\right\|^{2}
$$

mostra que neste caso $c_{n}(X) \leq 1$. Logo para espaços normados

$$
n^{-1} b_{n}(X) \leq c_{n}(X) \leq 1
$$

Vamos estudar mais uma caracterização dos espaços B-convexos, agora na categoria de 
espaços normados.

Lema 2.22. Seja $X$ um espaço normado. Então $X$ é B-convexo se, e somente, se $\lim _{n \rightarrow \infty} c_{n}(X)=$ 0

Demonstração. Do comentário anterior é claro que se $\lim _{n \rightarrow \infty} c_{n}(X)=0$ então $X$ é Bconvexo. Reciprocamente, suponhamos que $X$ seja B-convexo. O objetivo será encontrar um $m \in \mathbb{N}$ tal que $c_{m}<1$. Com efeito, se existe tal $m$, para todo $n \in \mathbb{N}$ com $n \geq m^{k}$, temos $c_{n} \leq c_{m^{k}} \leq\left(c_{m}\right)^{k}$. Logo $\lim _{n \rightarrow \infty} c_{n}(X)=0$.

Assim, é suficiente demonstrar que se $c_{n}=1$ então $n^{-1} b_{n}=1$ para todo $n \in \mathbb{N}$. Como observamos anteriormente, se $c_{n}=1$, temos

$$
n^{2}=\sup \int_{0}^{1}\left\|\sum_{k=1}^{n} \gamma_{k}(t) x_{k}\right\|^{2} d t=\sup \frac{1}{2^{n}} \sum_{\epsilon_{k}= \pm 1}\left\|\sum_{k=1}^{n} \epsilon_{k} x_{k}\right\|^{2}
$$

onde o supremo é tomado sobre todos os $x_{1}, \ldots, x_{n} \in \mathfrak{B}_{X}$.

Por enquanto, fixemos $x_{1}, \ldots, x_{n} \in \mathfrak{B}_{X}$ e escolha uma sequência de sinais $\widetilde{\epsilon}=\left(\widetilde{\epsilon}_{k}\right)_{k=1}^{n}$ tal que

$$
\left\|\sum_{k=1}^{n} \widetilde{\epsilon}_{k} x_{k}\right\|=\min _{\epsilon_{k}= \pm 1}\left\|\sum_{k=1}^{n} \epsilon_{k} x_{k}\right\| .
$$

É claro que $\left\|\sum_{k=1}^{n} \widetilde{\epsilon}_{k} x_{k}\right\| \leq b_{n}$. Logo

$$
\begin{aligned}
\sum_{\epsilon_{k}= \pm 1}\left\|\sum_{k=1}^{n} \epsilon_{k} x_{k}\right\|^{2} & \leq\left(\sum_{\epsilon \neq \widetilde{\epsilon}}\left\|\sum_{k=1}^{n} \epsilon_{k} x_{k}\right\|^{2}\right)+\left\|\sum_{k=1}^{n} \widetilde{\epsilon}_{k} x_{k}\right\|^{2} \\
& \leq\left(2^{n}-1\right) n^{2}+b_{n}^{2}
\end{aligned}
$$

Tomando o supremo sobre todos os $x_{1}, \ldots, x_{n} \in \mathfrak{B}_{X}$ na expressão anterior, obtemos

$$
n^{2} \leq 2^{-n}\left(\left(2^{n}-1\right) n^{2}+b_{n}\right)
$$

Desenvolvendo esta fórmula achamos $n^{-1} b_{n} \geq 1$, assim devemos ter $n^{-1} b_{n}=1$.

Vejamos alguns exemplos.

Exemplo 1. Todo espaço de dimensão finita é B-convexo.

Seja $X$ um espaço normado com $\operatorname{dim} X<n$. Fixemos n vetores $x_{1}, \ldots, x_{n} \in \mathfrak{B}_{X}$ e tomemos escalares $a_{1}, \ldots, a_{n}$ não todos zero tais que $\sum_{i=1}^{n} a_{i} x_{i}=0$, podemos escolher os escalares de forma que $\sup _{1 \leq i \leq n}\left|a_{i}\right|=1$ e reais, pois no caso complexo é suficiente tomar a parte real. Escrevamos $\epsilon_{i}=\operatorname{sinal}\left(a_{i}\right)$ se $a_{i} \neq 0$ e $\epsilon_{i}=0$ em outro caso. Com isto, $\left|\epsilon_{i}-a_{i}\right| \leq 1$ e para algum $i \in\{1, \ldots, n\}, \epsilon_{i}-a_{i}=0$. Portanto 


$$
\begin{aligned}
n^{-1}\left\|\sum_{i=1}^{n} \epsilon_{i} x_{i}\right\| & =n^{-1}\left\|\sum_{i=1}^{n} a_{i} x_{i}+\sum_{i=1}^{n}\left(\epsilon_{i}-a_{i}\right) x_{i}\right\| \\
& \leq n^{-1} \sum_{i=1}^{n}\left|\epsilon_{i}-a_{i}\right| \\
& \leq n^{-1}(n-1)=1-n^{-1}
\end{aligned}
$$

Logo $n^{-1} b_{n}(X)<1$, o que prova que $X$ é B-convexo.

Exemplo 2. Todo espaço uniformemente convexo é B-convexo, em particular espaços com produto interno são B-convexos.

Lembremos que um espaço normado $X$ é uniformemente convexo, se para cada $\epsilon>0$, existe $\delta>0$ tal que para todo $x, y \in X \operatorname{com}\|x\|=\|y\|=1$ se $\|x-y\| \geq \epsilon$ então $\|x+y\|<2(1-\delta)$. Equivalentemente, $X$ é uniformemente convexo se para cada $\epsilon>0$ esxiste $\delta^{\prime}>0$ tal que se $x, y \in \mathfrak{B}_{X}$ com $\|x-y\|>\epsilon$, então $\|x+y\| \leq 2\left(1-\delta^{\prime}\right)$. Para ver que esta propriedade implica ser B-convexo, seja $\delta^{\prime}>0$ tomado da definição quando $\epsilon=1$ e escrevamos $\theta=\min \left\{\delta^{\prime}, 1 / 2\right\}$. Fixemos $x, y \in \mathfrak{B}_{X}$. Então podemos ter $\|x-y\| \leq 1=2(1-1 / 2) \leq 2(1-\theta)$ ou $\|x-y\|>1$ em tal caso $\|x+y\| \leq 2\left(1-\delta^{\prime}\right) \leq 2(1-\theta)$. Com isto, $2^{-1} b_{2}(X)<1$, assim $X$ é B-convexo. Exemplos de espaços uniformemente convexos são espaços com produto interno. Com efeito, pela caracterização

$$
\|x+y\|^{2}+\|x-y\|^{2}=2\left(\|x\|^{2}+\|y\|^{2}\right)
$$

se $\|x\|=\|y\|=1$, então $\|x+y\|^{2}+\|x-y\|^{2}=4$. Portanto, se $\|x-y\| \geq \epsilon$, temos $\|x+y\|^{2}=$ $4-\|x-y\|^{2} \leq 4-\epsilon^{2}$. Assim $\|x+y\| \leq 2(1-\delta)$ onde $\delta=1-\left(1-(\epsilon / 2)^{2}\right)^{1 / 2}$.

Concluímos que $\ell_{2}$ é B-convexo.

Exemplo 3. O espaço $\ell_{1}$ não é B-convexo.

Para cada $n \in \mathbb{N}$ se $\left(\epsilon_{i}\right)_{i=1}^{n}$ é uma escolha de $n$ sinais e $e_{1}, \ldots, e_{n}$ são os primeiros $n$ vetores da base canônica, temos $n^{-1}\left\|\sum_{i=1}^{n} \epsilon_{i} e_{i}\right\|_{1}=1$. Portanto, $n^{-1} b_{n}\left(\ell_{1}\right)=1$ e em consequência $\ell_{1}$ não é B-convexo.

Exemplo 4. Os espaços $\ell_{\infty}$ e $c_{0}$ não são B-convexos.

Fixemos $n \in \mathbb{N}$, definamos $n$ sequências $x_{1}, \ldots, x_{n} \in c_{0}$ da seguinte forma

$$
x_{i}(j)=\gamma_{i}\left(t_{j}\right), \quad t_{j} \in\left(\frac{j-1}{2^{n}}, \frac{j}{2^{n}}\right), \quad j=1, \ldots, 2^{n}
$$

e $x_{i}(j)=0$ para $j>2^{n}$. Onde $\gamma_{1}, \ldots, \gamma_{n}$ são as primeiras $n$ funções de Rademacher. Pelo Lema (2.17) para cada sequência de $n$ sinais $\left(\epsilon_{i}\right)_{i=1}^{n}$ existe $j \in\left\{1, \ldots, 2^{n}\right\}$ tal que $x_{i}(j)=\epsilon_{i}$ para $i=1, \ldots, n$. Assim, a $j$-ésima coordenada de $\sum_{i=1}^{n} \epsilon_{i} x_{i}$ é $n$. Portanto $n^{-1}\left\|\sum_{i=1}^{n} \epsilon_{i} x_{i}\right\|_{\infty}=1$. Com isto, $n^{-1} b_{n}\left(\ell_{\infty}\right)=n^{-1} b_{n}\left(c_{0}\right)=1$. Concluímos que $\ell_{\infty}$ e $c_{0}$ não são B-convexos.

Agora vamos provar alguns lemas técnicos, para demonstrar que toda soma torcida de 
espaços de Banach B-convexos é um espaço de Banach B-convexo.

Teorema 2.23. Seja $X$ um espaço quase-normado tal que $\sup n^{-1} a_{n}<\infty$. Então 0 possui uma vizinhança convexa e limitada.

Demonstração. Vamos mostrar que a envoltória convexa do conjunto $\mathfrak{B}_{X}$ é limitada.

Seja $C>0$ uma constante tal que $a_{n} \leq C n$ para todo $n \in \mathbb{N}$. Considere $x_{1}, \ldots, x_{n} \in \mathfrak{B}_{X}$ e $\alpha_{1}, \ldots, \alpha_{n}$ números reais positivos tais que $\sum_{i=1}^{n} \alpha_{i}=1$. Então:

$$
\left\|\sum_{i=1}^{n}\left[m \alpha_{i}\right] x_{i}\right\| \leq C\left(\sum_{i=1}^{n}\left[m \alpha_{i}\right]\right) .
$$

Como $\sum_{i=1}^{n}\left[m \alpha_{i}\right] \leq \sum_{i=1}^{n} m \alpha_{i}=m$, obtemos

$$
\left\|\sum_{i=1}^{n} \frac{\left[m \alpha_{i}\right]}{m} x_{i}\right\| \leq C .
$$

Também observamos que $\lim _{m \rightarrow \infty}\left[m \alpha_{i}\right] / m=\alpha_{i}$ pois $(m-1) \alpha_{i} \leq\left[m \alpha_{i}\right] \leq m \alpha_{i}$. Logo passando ao limite(Observação (1.38))

$$
\left\|\sum_{i=1}^{n} \alpha_{i} x_{i}\right\| \leq C K
$$

onde $K$ é o módulo de concavidade da quase-norma.

Lema 2.24. Seja $X$ um espaço quase-normado tal que $\lim n^{-1} b_{n}=0$. Então existe $p>1$ tal que $\sup n^{-1 / p} b_{n}<\infty$

Demonstração. Por hipótese existe um $m \in \mathbb{N}$ tal que $m^{-1} b_{m}<1$. Considerando a função $m^{r}$ com $r>0$, podemos encontrar um $p>1$ que satisfaz $m^{-1 / p} b_{m}<1$ e usando o Lema (2.20) temos $m^{-k / p} b_{m^{k}} \leq\left(m^{-1 / p} b_{m}\right)^{k}$ para todo inteiro positivo $k$. Logo para qualquer $n \in \mathbb{N}$, se $m^{k} \leq n<m^{k+1}$

$$
\begin{aligned}
n^{-1 / p} b_{n} & \leq m^{-k / p} b_{m^{k+1}}=m^{1 / p}\left(m^{-(k+1) / p} b_{m^{k+1}}\right) \\
& \leq m^{1 / p}\left(m^{-1 / p} b_{m}\right)^{k+1}
\end{aligned}
$$

e assim $\lim _{n \rightarrow \infty} n^{-1 / p} b_{n}=0$ o que permite concluir que $n^{-1 / p} b_{n}$ é limitada.

Lema 2.25. Em um espaço quase-normado $X$, se $\lim n^{-1} b_{n}=0$ então $\sup n^{-1} a_{n}<\infty$

Demonstração. Primeiro observemos que é suficiente demonstrar que sup $2^{-n} a_{2^{n}}<\infty$. Com efeito, suponhamos que exista $C>0$ tal que $2^{-k} a_{2^{k}} \leq C$ para todo $k \in \mathbb{N}$. Sejam $n \in \mathbb{N}$ e $x_{1}, \ldots, x_{n} \in X$ com $\left\|x_{i}\right\| \leq 1$, se $2^{k} \leq n<2^{k+1}$ então

$$
\begin{aligned}
\frac{1}{2} n^{-1}\left\|x_{1}+\cdots+x_{n}\right\| & \leq 2^{-(k+1)}\left\|x_{1}+\cdots+x_{n}+0+\cdots+0\right\| \\
& \leq 2^{-(k+1)} a_{2^{k+1}}
\end{aligned}
$$


Assim $n^{-1} a_{n} \leq 2 C$. Isto mostra que o lema fica provado se $\left(n^{-1} a_{n}\right)^{r}$ é limitado para $n=2^{k}$ e algum $r>0$ fixo.

Sejam $d>0$ e $p>1$ constantes, obtidas pelo lema (2.24), tais que $b_{n} \leq d n^{1 / p}$, isto é $n^{-1} b_{n} \leq d n^{1 / p-1}$. Escrevamos $1 / q=1-1 / p>0$, tomando $n$ da forma $2^{k}$ observamos que para qualquer $r>0$

$$
\left(2^{-k} b_{2^{k}}\right)^{r} \leq d^{r} 2^{-k r / q}
$$

de onde

$$
\sum_{k=0}^{n}\left(2^{-k} b_{2^{k}}\right)^{r} \leq d^{r} \sum_{k=0}^{\infty} 2^{-k r / q}=C
$$

para todo $n \in \mathbb{N}$ (é claro que $C<\infty$ ).

Vamos considerar um $r>0$ tal que a quase-norma $\|$.$\| em X$ seja equivalente a uma $r$-norma( i.é uma quase-norma que satisfaz $\left.\|x+y\|^{r} \leq\|x\|^{r}+\|y\|^{r}\right)$.

Agora sejam $\left\{x_{1}, \ldots, x_{2 n}\right\} 2$ n vetores com $\left\|x_{i}\right\| \leq 1$ e uma escolha de sinais $\epsilon_{i}= \pm 1$ tal que

$$
\left\|\sum_{i=1}^{2 n} \epsilon_{i} x_{i}\right\| \leq b_{2 n}
$$

Definamos os conjuntos $A=\left\{i \leq 2 n: \epsilon_{i}=+1\right\}$ e $B=\left\{i \leq 2 n: \epsilon_{i}=-1\right\}$. Podemos supor que $|A| \leq n$. Assim

$$
\sum_{i=1}^{2 n} x_{i}=2 \sum_{i \in A} x_{i}-\sum_{i=1}^{2 n} \epsilon_{i} x_{i}
$$

Logo

$$
\begin{aligned}
\left\|\sum_{i=1}^{2 n} x_{i}\right\|^{r} & \leq 2^{r}\left\|\sum_{i \in A} x_{i}\right\|^{r}+b_{2 n}^{r} \\
& \leq 2^{r} a_{n}^{r}+b_{2 n}^{r} .
\end{aligned}
$$

Portanto $a_{2 n}^{r} \leq 2^{r} a_{n}^{r}+b_{2 n}^{r}$. Multiplicando por $(2 n)^{-r}$

$$
(2 n)^{-r} a_{2 n}^{r} \leq n^{-r} a_{n}^{r}+(2 n)^{-r} b_{2 n}^{r}
$$

Daqui, temos

$$
2^{-(n+1) r} a_{2^{n+1}}^{r}-2^{-n r} a_{2^{n}}^{r} \leq 2^{-(n+1) r} b_{2^{n+1}}^{r} .
$$


Logo

$$
\begin{aligned}
2^{-(n+1) r} a_{2^{n+1}}^{r}-a_{1}^{r} & =\sum_{k=0}^{n}\left(2^{-(k+1) r} a_{2^{k+1}}^{r}-2^{-k r} a_{2^{k}}^{r}\right) \\
& \leq \sum_{k=0}^{n} 2^{-(k+1) r} b_{2^{k+1}}^{r} \leq C
\end{aligned}
$$

para todo $n \in \mathbb{N}$, onde a última desigualdade é garantida pela equação (2.1). Isto implica que $\sup _{k \in \mathbb{N}}\left(2^{-k} a_{2^{k}}\right)^{r}<\infty$, e assim a prova do lema fica completa.

Teorema 2.26. Sejam $X$ um espaço quase-normado e $Y$ um subespaço fechado de $X$ tal que $X / Y$ e $Y$ sejam isomorfos a espaços de Banach B-convexos. Então $X$ é B-convexo.

Demonstração. Seja $K$ um módulo de concavidade da quase-norma em $X$. Lembremos da definição (2.19) que para qualquer espaço localmente limitado $Z, c_{n}(Z)$ é a menor constante $c_{n}$ tal que

$$
\frac{1}{2^{n}} \sum_{\epsilon_{i}= \pm 1}\left\|\sum_{i=1}^{n} \epsilon_{i} x_{i}\right\|^{2} \leq n c_{n}^{2} \sum_{i=1}^{n}\left\|x_{i}\right\|^{2}
$$

para todo $x_{1}, \ldots, x_{n} \in Z$.

Pelo lema sobre B-convexidade em espaços normados(Lema $((2.22)))$ se $Z$ é isomorfo a um espaço de Banach B-convexo, então $\lim _{n \rightarrow \infty} c_{n}(Z)=0$ (pois passando a una quase-norma equivalente não altera esta propriedade). Assim, neste caso $\lim c_{n}(Y)=\lim c_{n}(X / Y)=0$.

Sejam $\left\{x_{i j}: 1 \leq i \leq m, 1 \leq j \leq n\right\}$ um conjunto de $n m$ vetores em $X$. Denotemos por $\theta_{i j}(1 \leq i \leq m, 1 \leq j \leq n)$ as mn primeiras funções de Rademacher em $[0,1]$. Então, pelo Lema (2.18)-(a)

$$
\frac{1}{2^{n m}} \sum_{\epsilon_{i j}= \pm 1}\left\|\sum_{i, j} \epsilon_{i j} x_{i j}\right\|^{2}=\int_{0}^{1}\left\|\sum_{i=1}^{m} \sum_{j=1}^{n} \theta_{i j}(t) x_{i j}\right\|^{2} d t
$$

Definamos por $\varphi_{1}, \ldots, \varphi_{m}$ as primeiras $m$ funções de Rademacher em [0,1], e para cada $i=1, \ldots, m$ seja $u_{i}(t):=\sum_{j=1}^{n} \theta_{i j} x_{i j}, t \in[0,1]$. Também pelo Lema (2.18)-(b)

$$
\begin{aligned}
\int_{0}^{1}\left\|\sum_{i=1}^{m} \sum_{j=1}^{n} \theta_{i j}(t) x_{i j}\right\|^{2} d t & =\int_{0}^{1} \int_{0}^{1}\left\|\sum_{i=1}^{m} \sum_{j=1}^{n} \varphi_{i}(s) \theta_{i j}(t) x_{i j}\right\|^{2} d s d t \\
& =\int_{0}^{1} \int_{0}^{1}\left\|\sum_{i=1}^{m} \varphi_{i}(s) u_{i}(t)\right\|^{2} d s d t
\end{aligned}
$$

Seja $A(t)=\left(\int_{0}^{1}\left\|\sum_{i=1}^{m} \varphi_{i}(s) u_{i}(t)\right\|^{2} d s\right)^{1 / 2}$.

A imagem da função $u_{i}$ é um conjunto finito de vetores de $X$, assim dado $t \in[0,1]$ existe 
$v_{i}(t) \in Y$ tal que

$$
\left\|u_{i}(t)+v_{i}(t)\right\| \leq 2\left\|\pi\left(u_{i}(t)\right)\right\|
$$

onde $\pi: X \rightarrow X / Y$ é a aplicação quociente. Portanto

$$
\begin{aligned}
\left\|v_{i}(t)\right\| & \leq K\left(\left\|u_{i}(t)+v_{i}(t)\right\|+\left\|u_{i}(t)\right\|\right) \\
& \leq 3 K\left\|u_{i}(t)\right\|
\end{aligned}
$$

Observamos

$$
\left\|\sum_{i=1}^{m} \varphi_{i}(s) u_{i}(t)\right\| \leq K\left(\left\|\sum_{i=1}^{m} \varphi_{i}(s)\left(u_{i}(t)+v_{i}(t)\right)\right\|+\left\|\sum_{i=1}^{m} \varphi_{i}(s) v_{i}(t)\right\|\right)
$$

Logo pela desigualdade triangular em $L_{2}$ e a definição de $c_{n}(Z)$

$$
\begin{aligned}
A(t) & \leq K\left(\int_{0}^{1}\left\|\sum_{i=1}^{m} \varphi_{i}(s)\left(u_{i}(t)+v_{i}(t)\right)\right\|^{2} d s\right)^{1 / 2}+K\left(\int_{0}^{1}\left\|\sum_{i=1}^{m} \varphi_{i}(s) v_{i}(t)\right\|^{2} d s\right)^{1 / 2} \\
& \leq K \sqrt{m} c_{m}(X)\left(\sum_{i=1}^{m}\left\|u_{i}(t)+v_{i}(t)\right\|^{2}\right)^{1 / 2}+K \sqrt{m} c_{m}(Y)\left(\sum_{i=1}^{m}\left\|v_{i}(t)\right\|^{2}\right)^{1 / 2} \\
& \leq 2 K \sqrt{m} c_{m}(X)\left(\sum_{i=1}^{m}\left\|\pi\left(u_{i}(t)\right)\right\|^{2}\right)^{1 / 2}+3 K^{2} \sqrt{m} c_{m}(Y)\left(\sum_{i=1}^{m}\left\|u_{i}(t)\right\|^{2}\right)^{1 / 2}
\end{aligned}
$$

Usando de novo a desigualdade triangular para funções em $L_{2}$

$$
\begin{aligned}
\left(\int_{0}^{1} A^{2}(t) d t\right)^{1 / 2} & \leq 2 K \sqrt{m} c_{m}(X)\left(\sum_{i=1}^{m} \int_{0}^{1}\left\|\pi\left(u_{i}(t)\right)\right\|^{2}\right)^{1 / 2} \\
& +3 K^{2} \sqrt{m} c_{m}(Y)\left(\sum_{i=1}^{m} \int_{0}^{1}\left\|u_{i}(t)\right\|^{2}\right)^{1 / 2}
\end{aligned}
$$

Observemos que $\pi\left(u_{i}(t)\right)=\sum_{j=1}^{n} \theta_{i j}(t) \pi\left(x_{i j}\right)$. E assim:

$$
\sum_{i=1}^{m} \int_{0}^{1}\left\|\sum_{i=1}^{m} \theta_{i j}(t) \pi\left(x_{i j}\right)\right\|^{2} d t \leq \sum_{i=1}^{m} c_{n}(X / Y)\left(\sum_{j=1}^{n}\left\|\pi\left(x_{i j}\right)\right\|^{2}\right)^{1 / 2}
$$

Usando a desigualdade anterior vamos limitar os termos da desigualdade (2.2). Para o primeiro: 


$$
\begin{aligned}
\left(\sum_{i=1}^{m} \int_{0}^{1}\left\|\pi\left(u_{i}(t)\right)\right\|^{2}\right)^{1 / 2} & =\left(\sum_{i=1}^{m} \int_{0}^{1}\left\|\sum_{i=1}^{m} \theta_{i j}(t) \pi\left(x_{i j}\right)\right\|^{2} d t\right)^{1 / 2} \\
& \leq\left(\sum_{i=1}^{m} n c_{n}^{2}(X / Y)\left(\sum_{j=1}^{n}\left\|\pi\left(x_{i j}\right)\right\|^{2}\right)\right)^{1 / 2} \\
& =\sqrt{n} c_{n}(X / Y)\left(\sum_{i=1}^{m} \sum_{j=1}^{n}\left\|\pi\left(x_{i j}\right)\right\|^{2}\right)^{1 / 2}
\end{aligned}
$$

Agora o segundo termo:

$$
\begin{aligned}
\left(\sum_{i=1}^{m} \int_{0}^{1}\left\|u_{i}(t)\right\|^{2}\right)^{1 / 2} & =\left(\sum_{i=1}^{m} \int_{0}^{1}\left\|\sum_{j=1}^{n} \theta_{i j}(t) x_{i j}\right\|^{2} d t\right)^{1 / 2} \\
& \leq\left(\sum_{i=1}^{m} n c_{n}^{2}(X)\left(\sum_{j=1}^{n}\left\|x_{i j}\right\|^{2}\right)\right)^{1 / 2} \\
& =\sqrt{n} c_{n}(X)\left(\sum_{i=1}^{m} \sum_{j=1}^{n}\left\|x_{i j}\right\|^{2}\right)^{1 / 2}
\end{aligned}
$$

Assim, usando as equações (2.2), (2.3) e (2.4) obtemos

$$
\begin{aligned}
\left(\frac{1}{2^{n m}} \sum_{\epsilon_{i j}= \pm 1}\left\|\sum_{i, j} \epsilon_{i j} x_{i j}\right\|^{2}\right)^{1 / 2} & =\left(\int_{0}^{1} A^{2}(t) d t\right)^{1 / 2} \\
& \leq K \sqrt{m} \sqrt{n}\left(2 c_{m}(X) c_{n}(X / Y)+3 K c_{m}(Y) c_{n}(X)\right)\left(\sum_{i, j}\left\|x_{i j}\right\|^{2}\right)^{1 / 2}
\end{aligned}
$$

o que permite concluir que

$$
c_{m n}(X) \leq K\left(2 c_{m}(X) c_{n}(X / Y)+3 K c_{m}(Y) c_{n}(X)\right)
$$

Em particular

$$
c_{m^{2}}(X) \leq\left(2 K c_{m}(X / Y)+3 K^{2} c_{m}(Y)\right) c_{m}(X)
$$

Pela hipótese $\lim _{m \rightarrow \infty} 2 K c_{m}(X / Y)+3 K^{2} c_{m}(Y)=0$. Assim usando a relação anterior, existe um $m \in \mathbb{N}$ tal que $c_{m}(X)=\alpha<1$. Do fato que $m^{-1} b_{m}(X) \leq c_{m}(X)<1$ concluímos que $X$ é B-convexo.

Teorema 2.27. Toda soma torcida de espaços de Banach B-convexos é um espaço de Banach B-convexo. 
Demonstração. Suponhamos que $X$ seja uma soma torcida de espaços de Banach B-convexos. Pelo teorema anterior, $X$ é B-convexo. Resulta dos teoremas (2.25) e (2.23) que $0 \in X$ possui uma vizinhança convexa limitada, como foi estudado no capítulo 1( Teorema (1.32)) essa é uma condição necessária e suficiente para que um espaço seja normalizável. Também $X$ deve ser completo (Teorema (2.11)). Em consequência $X$ é um espaço de Banach B-convexo.

\subsection{Simplificações na construção de somas torcidas}

Vamos mostrar duas simplificações para construir funções quase-lineares o que significa simplificações na construção de somas torcidas. A primeira nos permite considerar funções definidas num subespaço denso, e a segunda elimina a exigência da homogenidade.

Teorema 2.28. Sejam $X$ e $Y$ F-espaços quase-normados e $Y_{0}$ um subespaço denso de $Y$. Se $F_{0}: Y_{0} \rightarrow X$ é uma aplicação quase-linear, então existe uma aplicação quase-linear $F: Y \rightarrow X$ tal que $F(y)=F_{0}(y)$, para todo $y \in Y_{0}$

Demonstração. Considere o espaço $X \oplus_{F_{0}} Y_{0}$ com a quase norma

$$
\|(x, y)\|=\|y\|+\left\|x-F_{0}(y)\right\|
$$

Seja $Z$ um completamento deste espaço. Defina $j: X \rightarrow X \oplus_{F_{0}} Y_{0}$ como sendo $j(x)=(x, 0)$ e $\hat{q}: X \oplus_{F_{0}} Y_{0} \rightarrow Y$ por $\hat{q}(x, y)=y$. Sabemos que $\hat{q}$ é contínua e sobrejetora, então existe uma função $q: Z \rightarrow Y$ contínua que estende $\hat{q}$. Vamos demonstrar que a sequência curta

$$
0 \rightarrow X \stackrel{j}{\rightarrow} Z \stackrel{q}{\rightarrow} Y \rightarrow 0
$$

é exata. Para isto, só falta verificar que $j(X)=\operatorname{ker} q$. É claro que $j(X) \subseteq$ ker $q$ pois $q(j(x))=q(x, 0)=\hat{q}(x, 0)=0$.

Seja $z \in \operatorname{ker} q$, então $z=\lim \left(x_{n}, y_{n}\right)$ onde $\left(x_{n}, y_{n}\right) \in X \oplus_{F_{0}} Y_{0}$. Assim $0=q(z)=$ $\lim q\left(x_{n}, y_{n}\right)=\lim \hat{q}\left(x_{n}, y_{n}\right)=\lim y_{n}$, isto implica que $\lim \left(F_{0}\left(y_{n}\right), y_{n}\right)=0$, pois

$$
\left\|\left(F_{0}\left(y_{n}\right), y_{n}\right)\right\|=\left\|y_{n}\right\|+\left\|F_{0}\left(y_{n}\right)-F_{0}\left(y_{n}\right)\right\|=\left\|y_{n}\right\|
$$

Portanto, $\lim \left(x_{n}-F_{0}\left(y_{n}\right), 0\right)=z$. Observemos que

$$
\left\|\left(x_{n}-F_{0}\left(y_{n}\right)\right)-\left(x_{m}-F_{0}\left(y_{m}\right)\right)\right\|_{X} \leq\left\|\left(x_{n}-F_{0}\left(y_{n}\right), 0\right)-\left(x_{m}-F_{0}\left(y_{m}\right), 0\right)\right\|_{F_{0}}
$$

Logo $\left(x_{n}-F_{0}\left(y_{n}\right)\right)_{n}$ é de Cauchy em $X$ e em consequência convergente a um $x_{0} \in X$. Então

$$
j\left(x_{0}\right)=\lim j\left(x_{n}-F_{0}\left(y_{n}\right)\right)=\lim \left(x_{n}-F_{0}\left(y_{n}\right), 0\right)=z
$$

o que mostra que $\operatorname{ker} q \subseteq j(X)$.

Logo $Z$ é equivalente a $X \oplus_{H} Y$ para alguma aplicação quase-linear $H: Y \rightarrow X$. Seja 
$T: Z \rightarrow X \oplus_{H} Y$ um operador contínuo tal que o diagrama

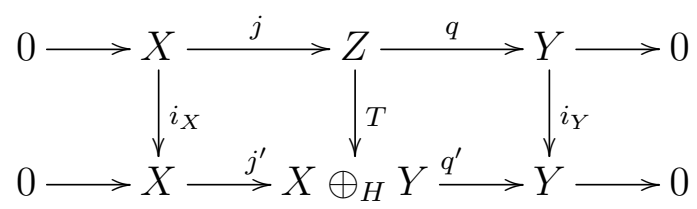

seja comutativo. Segue da demonstração do teorema 1.3, que $T \mid X \oplus_{F_{0}} Y_{0}$ tem a forma

$$
T(x, y)=(x+A y, y)
$$

onde $A: Y_{0} \rightarrow X$ é linear e tal que

$$
\left\|F_{0}(y)-H(y)+A(y)\right\| \leq\|T\|\|y\|, \quad y \in Y_{0}
$$

Consideremos pelo Lema (1.5) uma extenção linear de $A$ a todo $Y$, definamos

$$
F(y)=\left\{\begin{array}{rrr}
H(y)-A(y) & \text { se } & y \notin Y_{0} \\
F_{0}(y) & \text { se } & y \in Y_{0}
\end{array}\right.
$$

Da fórmula anterior é claro que $F(\lambda y)=\lambda F(y)$ para todo $\lambda \in \mathbb{K}$ e $y \in Y$. Para mostrar que $F$ é um operador quase-linear, temos que achar uma constante $C$ tal que

$$
\left\|F\left(y_{1}+y_{2}\right)-F\left(y_{1}\right)-F\left(y_{2}\right)\right\| \leq C\left(\left\|y_{1}\right\|+\left\|y_{2}\right\|\right)
$$

para todo $y_{1}, y_{2} \in Y$. Para isto, devemos verificar a desigualdade anterior em cada um dos seguintes casos:

(a) $y_{1}, y_{2} \in Y_{0}$;

(b) $y_{1} \in Y_{0}, y_{2} \notin Y_{0}$;

(c) $y_{1} \notin Y_{0}, y_{2} \notin Y_{0}$.

O caso (a) é claro. Para o caso (b), observemos que $y_{1}+y_{2} \notin Y_{0}$ e então

$$
\begin{aligned}
\left\|F\left(y_{1}+y_{2}\right)-F\left(y_{1}\right)-F\left(y_{2}\right)\right\| & =\left\|H\left(y_{1}+y_{2}\right)-A\left(y_{1}+y_{2}\right)-F_{0}\left(y_{1}\right)-H\left(y_{2}\right)+A\left(y_{2}\right)\right\| \\
& \leq K\left\|H\left(y_{1}+y_{2}\right)-H\left(y_{2}\right)-H\left(y_{1}\right)\right\|+K\left\|F_{0}\left(y_{1}\right)-H\left(y_{1}\right)-A\left(y_{1}\right)\right\| \\
& \leq K M_{H}\left(\left\|y_{1}\right\|+\left\|y_{2}\right\|\right)+K\|T\|\left\|y_{1}\right\| \\
& \leq\left(K M_{H}+K\|T\|\right)\left(\left\|y_{1}\right\|+\left\|y_{2}\right\|\right)
\end{aligned}
$$

onde $K$ é um módulo da quase-norma em $X$ e $M_{H}$ é uma constante que depende do operador quase-linear $H$. A prova no caso (c) é similar. Concluímos que $F$ é um operador quaselinear. 
Definição 2.29. Seja $Y$ um espaço quase-normado. Uma função $f: Y \rightarrow X$ chama-se quase-aditiva se

(a) $\left\|f\left(y_{1}+y_{2}\right)-f\left(y_{1}\right)-f\left(y_{2}\right)\right\| \leq k\left(\left\|y_{1}\right\|+\left\|y_{2}\right\|\right) \quad y_{1}, y_{2} \in Y$

(b) $\lim _{t \rightarrow 0} f(t y)=0, \quad y \in Y$

(c) $f(\lambda y)=\lambda f(y), \quad y \in Y$ e $\lambda \in \mathbb{K} \operatorname{com}|\lambda|=1$.

Onde $k$ é uma constante em (a). Dizemos que $f$ é quase-aditiva de ordem $k$.

Lema 2.30. Sejam $X$ e $Y$ espaços quase-normados. Então existem constantes positivas $r e$ $L$ tais que, se $f: Y \rightarrow X$ é uma função quase-aditiva de ordem $K$, então

$$
\left\|f\left(\sum_{i=1}^{n} y_{i}\right)-\sum_{i=1}^{n} f\left(y_{i}\right)\right\| \leq K L\left(\sum_{i=1}^{n}\left\|y_{i}\right\|^{r}\right)^{1 / r}
$$

para quaisquer $y_{1}, \ldots, y_{n} \in Y$.

Demonstração. Suponhamos $\left(X,\|\cdot\|_{X}\right)$ e $\left(Y,\|\cdot\|_{Y}\right)$ são espaços quase-normados. Pela observação (1.44) do teorema de Aoki-Rolewicz, existem quase-normas $|\cdot|_{X}$ e $|\cdot|_{Y}$ equivalentes a $\|\cdot\|_{X}$ e $\|\cdot\|_{Y}$, respetivamente, e um $0<p \leq 1$ tais que

$$
\begin{gathered}
\left|x_{1}+x_{2}\right|_{X}^{p} \leq\left|x_{1}\right|_{X}^{p}+\left|x_{2}\right|_{X}^{p}, \quad x_{1}, x_{2} \in X \\
\left|y_{1}+y_{2}\right|_{Y}^{p} \leq\left|y_{1}\right|_{Y}^{p}+\left|y_{2}\right|_{Y}^{p}, \quad y_{1}, y_{2} \in Y
\end{gathered}
$$

e para constantes positivas $C, D<\infty$

$$
\begin{gathered}
\|x\|_{X} \leq|x|_{X} \leq C\|x\|_{X}, \quad x \in X \\
\|y\|_{Y} \leq|y|_{Y} \leq D\|y\|_{Y}, \quad y \in Y
\end{gathered}
$$

Portanto

$$
\begin{aligned}
\left|f\left(y_{1}+y_{2}\right)-f\left(y_{1}\right)-f\left(y_{2}\right)\right|_{X} & \leq C\left\|f\left(y_{1}+y_{2}\right)-f\left(y_{1}\right)-f\left(y_{2}\right)\right\|_{X} \\
& \leq C K\left(\left|y_{1}\right|_{Y}+\left|y_{2}\right|_{Y}\right) \\
& \leq C K\left(\left|y_{1}\right|_{Y}^{p}+\left|y_{2}\right|_{Y}^{p}\right)^{1 / p}
\end{aligned}
$$

pois $(a+b)^{p} \leq a^{p}+b^{p}$ se $a, b \geq 0$. Por indução, vamos provar que para todo $n \in \mathbb{N}$

$$
\left|f\left(\sum_{i=1}^{n} y_{i}\right)-\sum_{i=1}^{n} f\left(y_{i}\right)\right|_{X} \leq C K\left(\sum_{i=1}^{n} i\left|y_{i}\right|_{Y}^{p}\right)^{1 / p}
$$

Com efeito, o caso $n=2$ segue do comentário anterior. Agora suponhamos que a fórmula 
(2.5) seja válida para $n-1$. Então

$$
\begin{aligned}
\left|f\left(\sum_{i=1}^{n} y_{i}\right)-\sum_{i=1}^{n} f\left(y_{i}\right)\right|_{X}^{p} & =\left|f\left(\sum_{i=2}^{n} y_{i}\right)-\sum_{i=2}^{n} f\left(y_{i}\right)+f\left(\sum_{i=1}^{n} y_{i}\right)-f\left(\sum_{i=2}^{n} y_{i}\right)-f\left(x_{1}\right)\right|_{X} \\
& \leq\left|f\left(\sum_{i=2}^{n} y_{i}\right)-\sum_{i=2}^{n} f\left(y_{i}\right)\right|_{X}^{p}+\left|f\left(\sum_{i=1}^{n} y_{i}\right)-f\left(\sum_{i=2}^{n} y_{i}\right)-f\left(x_{1}\right)\right|_{X} \\
& \leq C^{p} K^{p}\left(\sum_{i=2}^{n}(i-1)\left|y_{i}\right|_{Y}^{p}\right)+C^{p} K^{p}\left(\left|\sum_{i=2}^{n} y_{i}\right|_{Y}+\left|y_{1}\right|_{Y}\right)^{p} \\
& \leq C^{p} K^{p}\left(\sum_{i=2}^{n}(i-1)\left|y_{i}\right|_{Y}^{p}+\left|\sum_{i=2}^{n} y_{i}\right|_{Y}^{p}+\left|y_{1}\right|_{Y}^{p}\right) \\
& \leq C^{p} K^{p}\left(\sum_{i=1}^{n} i\left|y_{i}\right|_{Y}^{p}\right)
\end{aligned}
$$

Escrevamos $r=p / 2$. Para $y_{1}, \ldots, y_{n} \in Y \operatorname{com}\left|y_{1}\right|_{Y} \geq\left|y_{2}\right|_{Y} \cdots \geq\left|y_{n}\right|_{Y}$

$$
\begin{aligned}
\sum_{i=1}^{n} i\left|y_{i}\right|_{Y}^{p} & =\sum_{i=1}^{n} i\left|y_{i}\right|_{Y}^{r}\left|y_{i}\right|_{Y}^{r} \\
& \leq \sum_{i=1}^{n}\left(\sum_{j=1}^{i}\left|y_{j}\right|_{Y}^{r}\right)\left|y_{i}\right|_{Y}^{r} \\
& \leq \sum_{i=1}^{n} \sum_{j=1}^{n}\left|y_{i}\right|_{Y}^{r}\left|y_{j}\right|_{Y}^{r}=\left(\sum_{i=1}^{n}\left|y_{i}\right|_{Y}^{r}\right)^{2}
\end{aligned}
$$

Assim

$$
\begin{aligned}
\left|f\left(\sum_{i=1}^{n} y_{i}\right)-\sum_{i=1}^{n} f\left(y_{i}\right)\right|_{X} & \leq C K\left(\sum_{i=1}^{n} i\left|y_{i}\right|_{Y}^{p}\right)^{1 / p} \\
& \leq\left(\sum_{i=1}^{n}\left|y_{i}\right|_{Y}^{r}\right)^{2 / p} \\
& =\left(\sum_{i=1}^{n}\left|y_{i}\right|_{Y}^{r}\right)^{1 / r}
\end{aligned}
$$

Mediante um reordenamento, a desigualdade é válida para todo $y_{1}, \ldots, y_{n} \in Y$, e em consequência

$$
\left\|f\left(\sum_{i=1}^{n} y_{i}\right)-\sum_{i=1}^{n} f\left(y_{i}\right)\right\| \leq C D K\left(\sum_{i=1}^{n}\left\|y_{i}\right\|^{r}\right)^{1 / r}
$$

Lema 2.31. Suponha $X$ um F-espaço quase-normado. Então existe uma constante $K_{X}$, que 
depende de $X$, tal que para toda $f: \mathbb{R} \rightarrow X$ quase-aditiva de ordem $K$, temos

$$
\|f(t)-t f(1)\| \leq K_{X} K \quad 0 \leq t \leq 1
$$

Demonstração. Vamos dividir a prova em vários passos.

PASSO 1. Podemos supor $f(1)=0$, pois em outro caso, aplicamos o lema para $g(t)=$ $f(t)-f(1)$ que é quase-aditiva de ordem $K$ e, satisfaz $g(1)=0$ :

$$
\begin{aligned}
g\left(t_{1}+t_{2}\right)-g\left(t_{1}\right)-g\left(t_{2}\right) & =\left(f\left(t_{1}+t_{2}\right)-\left(t_{1}+t_{2}\right) f(1)\right) \\
& \left.-\left(f\left(t_{1}\right)-t_{1} f(1)\right)-\left(f\left(t_{1}\right)-t_{1} f(1)\right)\right) \\
& =f\left(t_{1}+t_{2}\right)-f\left(t_{1}\right)-f\left(t_{2}\right)
\end{aligned}
$$

e assim $\left\|g\left(y_{1}+y_{2}\right)-g\left(y_{1}\right)-g\left(y_{2}\right)\right\| \leq k\left(\left\|y_{1}\right\|+\left\|y_{2}\right\|\right)$. Também

$$
\lim _{t \rightarrow 0} g(t y)=\lim _{t \rightarrow 0}(f(t y)-t f(1))=0
$$

e $g(-t)=f(-t)-t f(1)=-g(t)$.

PASSO 2. Aplicando o lema anterior com $Y=\mathbb{R}=Y_{0}$ e o Teorema (1.43), existem constantes positivas $r$ e $L$ tais que

$$
\begin{aligned}
\left\|f\left(\sum_{i=1}^{n} t_{i}\right)-\sum_{i=1}^{n} f\left(t_{i}\right)\right\| & \leq K L\left(\sum_{i=1}^{n}\left\|t_{i}\right\|^{r}\right)^{1 / r} \\
\left\|\sum_{i=1}^{n} x_{i}\right\| & \leq L\left(\sum_{i=1}^{n}\left\|x_{i}\right\|^{r}\right)^{1 / r}
\end{aligned}
$$

para todo $t_{1}, \ldots t_{n} \in \mathbb{R}$ e $x_{1}, \ldots x_{n} \in X$.

PASSO 3. O objetivo é mostrar que existe uma constante $K_{X}$ dependendo só do espaço $X$ tal que

$$
\|f(t)\| \leq K_{X} K \quad 0<t<1
$$

Observemos inicialmente que se $s \in[1,2)$ podemos escrever $s=\sum_{k=0}^{\infty} \epsilon_{k} 2^{-k}$ onde $\epsilon_{k}=0$ ou 1. Com efeito, tome $\epsilon_{0}=1$ e defina

$$
\epsilon_{k+1}=\left\{\begin{array}{rlc}
1, & \text { se } & s \geq \sum_{j=0}^{k} \epsilon_{j} 2^{-j}+2^{-(k+1)} \\
0 & \text { se } & s<\sum_{j=0}^{k} \epsilon_{j} 2^{-j}+2^{-(k+1)}
\end{array}\right.
$$

logo para $t \in(0,1)$ e $n \in \mathbb{N}$ tal que $2^{-n} \leq t<2^{-(n-1)}$ temos que

$$
2^{n} t=\sum_{k=0}^{\infty} \epsilon_{k} 2^{-k}
$$


isto é $t=2^{-n} \sum_{k=0}^{\infty} \epsilon_{k} 2^{-k}$ e definamos

$$
t_{m}=2^{-n} \sum_{k=0}^{m} \epsilon_{k} 2^{-k}
$$

Como $f$ é quase-aditiva, temos

$$
\left\|f(t)-f\left(t_{m}\right)-f\left(t-t_{m}\right)\right\| \leq K\left(t_{m}+\left(t-t_{m}\right)\right) \leq K
$$

No PASSO 4, vamos mostrar que existe uma constante $C>0$ tal que para todo $m \in \mathbb{N}$

$$
\left\|f\left(t_{m}\right)\right\| \leq K C
$$

assumindo isto e usando o PASSO 2, observamos que

$$
\begin{aligned}
\|f(t)\| & =\left\|\left(f(t)-f\left(t_{m}\right)-f\left(t-t_{m}\right)\right)+f\left(t_{m}\right)+f\left(t-t_{m}\right)\right\| \\
& \leq L\left(\left\|f(t)-f\left(t_{m}\right)-f\left(t-t_{m}\right)\right\|^{r}+\left\|f\left(t_{m}\right)\right\|^{r}+\left\|f\left(t-t_{m}\right)\right\|^{r}\right)^{1 / r} \\
& \leq L\left(K^{r}+(K C)^{r}+\left\|f\left(t-t_{m}\right)\right\|^{r}\right)^{1 / r} \\
& =L K\left(1+C^{r}+K^{-r}\left\|f\left(t-t_{m}\right)\right\|^{r}\right)^{1 / r}
\end{aligned}
$$

Pela definição de $f$ é claro que se $m \rightarrow \infty$ então $f\left(t-t_{m}\right) \rightarrow 0$. Logo

$$
\|f(t)\| \leq L\left(1+C^{r}\right)^{1 / r} K=K_{X} K
$$

PASSO 4. Vamos provar a afirmação usada no passo anterior. Observamos pela quaseaditividade de $f$, que

$$
\left\|f\left(2^{-n}\right)-2 f\left(2^{-(n+1)}\right)\right\| \leq K\left(2^{-(n+1)}+2^{-(n+1)}\right)=K 2^{-n}
$$

Portanto

$$
\left\|2^{n} f\left(2^{-n}\right)-2^{n+1} f\left(2^{-(n+1)}\right)\right\| \leq K
$$

Também temos

$$
\begin{aligned}
2^{n} f\left(2^{-n}\right) & =2^{n} f\left(2^{-n}-2^{(n-1)} f\left(2^{-(n-1)}\right.\right. \\
& +2^{(n-1)} f\left(2^{-(n-1)}-2^{(n-2)} f\left(2^{-(n-2)}\right.\right. \\
& \vdots \\
& +2 f\left(2^{-1}\right)-2^{0} f\left(2^{0}\right)
\end{aligned}
$$

pois $f(1)=0$. Assim usando a equação (2.7) 


$$
\begin{aligned}
\left\|2^{n} f\left(2^{-n}\right)\right\| & \leq L\left(\sum_{i=0}^{n}\left\|2^{i} f\left(2^{-i}\right)-2^{i-1} f\left(2^{-(i-1)}\right)\right\|^{r}\right)^{1 / r} \\
& \leq L\left(\sum_{i=0}^{n} K^{r}\right)^{1 / r}=L K n^{1 / r}
\end{aligned}
$$

Agora

$$
\begin{aligned}
\left\|f\left(t_{m}\right)-\sum_{k=0}^{m} \epsilon_{k} f\left(2^{-(n+k)}\right)\right\| & =\left\|f\left(\sum_{k=0}^{m} \epsilon_{k} 2^{-(n+k)}\right)-\sum_{k=0}^{m} \epsilon_{k} f\left(2^{-(n+k)}\right)\right\| \\
& \leq K L\left(\sum_{k=0}^{m} \epsilon_{k} 2^{-r(n+k)}\right)^{1 / r} \\
& \leq K L\left(\sum_{k=0}^{\infty}\left(\frac{1}{2^{r}}\right)^{k}\right)^{1 / r} \\
& =K L\left(\frac{1}{1-2^{-r}}\right)^{1 / r}
\end{aligned}
$$

onde a primeira desigualdade é garantida pelo passo 2 .

Também pela equação (2.7)

$$
\begin{aligned}
\left\|\sum_{k=0}^{m} \epsilon_{k} f\left(2^{-(n+k)}\right)\right\| & \leq L\left(\sum_{i=0}^{n} \epsilon_{k}\left\|f\left(2^{-(n+k)}\right)\right\|^{r}\right)^{1 / r} \\
& \leq L\left(\sum_{k=0}^{m} \epsilon_{k} 2^{-(n+k) r}\left\|2^{-(n+k)} f\left(2^{-(n+k)}\right)\right\|^{r}\right)^{1 / r} \\
& \leq L\left(\sum_{k=0}^{m} \epsilon_{k} 2^{-(n+k) r}(L K)^{r}(n+k)\right)^{1 / r} \\
& =L^{2} K\left(\sum_{k=0}^{m} \epsilon_{k}(n+k) 2^{-(n+k) r}\right)^{1 / r}
\end{aligned}
$$


Finalmente, usando as desigualdades anteriores

$$
\begin{aligned}
\left\|f\left(t_{m}\right)\right\| & \leq L\left(\left\|f\left(t_{m}\right)-\sum_{k=0}^{m} \epsilon_{k} f\left(2^{-(n+k)}\right)\right\|^{r}+\left\|\sum_{k=0}^{m} \epsilon_{k} f\left(2^{-(n+k)}\right)\right\|^{r}\right)^{1 / r} \\
& \leq L\left((K L)^{r}\left(1-2^{-r}\right)^{-1}+\left(L^{2} K\right)^{r} \sum_{k=0}^{m} \epsilon_{k}(n+k) 2^{-(n+k) r}\right)^{1 / r} \\
& =K L^{2}\left(\left(1-2^{-r}\right)^{-1}+L^{r} \sum_{k=0}^{\infty} k 2^{-k r}\right)^{1 / r}=K C
\end{aligned}
$$

e a prova do lema agora está completa.

O lema anterior é o argumento principal do seguinte teorema que é uma 'fábrica' de funções quase-lineares.

Teorema 2.32. Sejam $X, Y$ F-espaços quase-normados e $Y_{0}$ um subespaço denso de $Y$. Considere $f: Y_{0} \rightarrow X$ uma aplicação quase-aditiva. Então a função $F: Y_{0} \rightarrow X$ definida por

$$
F(x)=\left\{\begin{array}{rrr}
\|x\| f\left(\frac{x}{\|x\|}\right) & \text { se } & x \neq 0 \\
0 & \text { se } & x=0
\end{array}\right.
$$

é quase-linear.

Demonstração. Tomemos primeiro $x \in Y_{0}, x \neq 0$ e definamos

$$
g(t)=f\left(t \frac{x}{\|x\|}\right), \quad t \in \mathbb{R} .
$$

Observamos que

$$
\begin{aligned}
\left\|g\left(t_{1}+t_{2}\right)-g\left(t_{1}\right)-g\left(t_{2}\right)\right\| & =\left\|f\left(\frac{t_{1}+t_{2}}{\|x\|} x\right)-f\left(\frac{t_{1}}{\|x\|} x\right)-f\left(\frac{t_{2}}{\|x\|} x\right)\right\| \\
& \leq K\left(\left\|\frac{t_{1} x}{\|x\|}\right\|+\left\|\frac{t_{2} x}{\|x\|}\right\|\right) \\
& =K\left(\left|t_{1}\right|+\left|t_{2}\right|\right)
\end{aligned}
$$

Logo $g$ é quase-aditiva de ordem $K$, para cada $x \in Y_{0}$. Suponhamos agora que $0<\|x\| \leq 1$. Temos pelo lema anterior

$$
\|g(\|x\|)-\| x\|g(1)\| \leq K_{X} K
$$

isto é $\|f(x)-\| x\left\|f\left(\frac{x}{\|x\|}\right)\right\| \leq K_{X} K$.

Denotemos por $C$ um módulo de concavidade da quase-norma em $X$. Então se $\|x\|+\|y\| \leq$ 
$1 / C$, temos $\|x+y\| \leq 1$ e assim

$$
\|f(x+y)-F(x+y)\| \leq K_{X} K
$$

Isto implica

$$
\begin{aligned}
& \|F(x+y)-F(x)-F(y)\|= \\
& \quad\|(F(x+y)-f(x+y))-(F(x)-f(x))-(F(y)-f(y))+(f(x+y)-f(x)-f(y))\| \\
& \quad \leq C\left(\|f(x+y)-f(x)-f(y)\|+3 C^{2} K_{X} K\right) \\
& \quad \leq C K\left(1+3 C^{2} K_{X}\right)=K^{*} .
\end{aligned}
$$

Logo aplicando o fato anterior a $\frac{x}{C(\|x\|+\|y\|)}$ e $\frac{y}{C(\|x\|+\|y\|)}$, obtemos

$$
\|F(x+y)-F(x)-F(y)\| \leq K^{*} C(\|x\|+\|y\|) .
$$

Para concluir que $F$ é quase-linear, seja $\lambda \in \mathbb{K}$

$$
\begin{aligned}
F(\lambda x)=\|\lambda x\| f\left(\frac{\lambda x}{\|\lambda x\|}\right) & =|\lambda|\|x\| f\left(\frac{\lambda}{|\lambda|} \frac{x}{\|x\|}\right) \\
& =\lambda\|x\| f\left(\frac{x}{\|x\|}\right) \\
& =\lambda F(x)
\end{aligned}
$$

Lema 2.33. Seja $\phi: \mathbb{R} \rightarrow \mathbb{C}$ uma função Lipschitz. Se L é a constante de Lipschitz de $\phi$, então

(a) Para todo $x_{1}, x_{2} \in \mathbb{R}$

$$
\left|\left(x_{1}+x_{2}\right) \phi\left(-\log \left|x_{1}+x_{2}\right|\right)-x_{1} \phi\left(-\log \left|x_{1}\right|\right)-x_{2} \phi\left(-\log \left|x_{2}\right|\right)\right| \leq L \log 2\left(\left|x_{1}\right|+\left|x_{2}\right|\right)
$$

(b) Para todo $z_{1}, z_{2} \in \mathbb{C}$

$$
\left|\left(z_{1}+z_{2}\right) \phi\left(-\log \left|z_{1}+z_{2}\right|\right)-z_{1} \phi\left(-\log \left|z_{1}\right|\right)-z_{2} \phi\left(-\log \left|z_{2}\right|\right)\right| \leq 8 L\left(\left|z_{1}\right|+\left|z_{2}\right|\right)
$$

Demonstração. (a). Suponhamos inicialmente $x_{1}, x_{2}>0$, então 


$$
\begin{aligned}
& \left|\phi\left(-\log \left(x_{1}+x_{2}\right)\right)-\frac{x_{1}}{x_{1}+x_{2}} \phi\left(-\log x_{1}\right)-\frac{x_{2}}{x_{1}+x_{2}} \phi\left(-\log x_{2}\right)\right| \\
& \leq \frac{x_{1}}{x_{1}+x_{2}}\left|\phi\left(-\log \left(x_{1}+x_{2}\right)\right)-\phi\left(-\log x_{1}\right)\right|+\frac{x_{2}}{x_{1}+x_{2}}\left|\phi\left(-\log \left(x_{1}+x_{2}\right)\right)-\phi\left(-\log x_{2}\right)\right| \\
& \leq L\left(\frac{x_{1}}{x_{1}+x_{2}} \log \left(\frac{x_{1}+x_{2}}{x_{1}}\right)+\frac{x_{2}}{x_{1}+x_{2}} \log \left(\frac{x_{1}+x_{2}}{x_{2}}\right)\right)
\end{aligned}
$$

$\leq L \log 2$

onde a última desigualdade resulta do fato que $t \log (1 / t)+(1-t) \log (1 /(1-t)) \leq \log 2$ $(0<t<1)$ (Isto porque $\log x$ é uma função côncava em $(0, \infty), \operatorname{logo} t \log \left(t^{-1}\right)+(1-$ t) $\left.\log \left((1-t)^{-1}\right) \leq \log \left(t t^{-1}+(1-t)(1-t)^{-1}\right)=\log 2\right)$. Portanto, fica provada a desigualdade de (a) no caso que $x_{1}$ e $x_{2}$ tenham o mesmo sinal. Agora suponhamos $x_{1}<0$ e $x_{2}>0$. Então se $x_{1}+x_{2}>0$ substituindo $x_{1}$ por $-x_{1}$ e $x_{2}$ por $x_{1}+x_{2}$

$$
\begin{aligned}
\mid\left(x_{1}+x_{2}\right) \phi & \left(-\log \left|x_{1}+x_{2}\right|\right)-x_{1} \phi\left(-\log \left|x_{1}\right|\right)-x_{2} \phi\left(-\log \left|x_{2}\right|\right) \mid \\
& =\left|x_{2} \phi\left(-\log x_{2}\right)+x_{1} \phi\left(-\log \left(-x_{1}\right)\right)-\left(x_{1}+x_{1}\right) \phi\left(-\log \left(x_{1}+x_{2}\right)\right)\right| \\
& \leq L \log 2\left(-x_{1}+\left(x_{1}+x_{2}\right)\right) \\
& \leq L \log 2\left(\left|x_{1}\right|+\left|x_{2}\right|\right) .
\end{aligned}
$$

Se $x_{1}+x_{2}<0$, então substituimos $x_{1}$ por $-x_{1}-x_{2}$ e deixamos $x_{2}$ fixo. Com isto,

$$
\begin{aligned}
\left|\left(x_{1}+x_{2}\right) \phi\left(-\log \left|x_{1}+x_{2}\right|\right)-x_{1} \phi\left(-\log \left|x_{1}\right|\right)-x_{2} \phi\left(-\log \left|x_{2}\right|\right)\right| \\
\quad=\left|-\left(x_{1}+x_{1}\right) \phi\left(-\log \left(-x_{1}-x_{2}\right)\right)+x_{2} \phi\left(-\log x_{2}\right)+x_{1} \phi\left(-\log \left(-x_{1}\right)\right)\right| \\
\leq L \log 2\left(-\left(x_{1}+x_{2}\right)+x_{2}\right) \\
\leq L \log 2\left(\left|x_{1}\right|+\left|x_{2}\right|\right) .
\end{aligned}
$$

O que completa a prova de (a).

Para demonstrar (b) escrevamos $z_{j}=x_{j}+i y_{j}, j=1,2$. Usando a parte (a), obtemos

$$
\begin{aligned}
& \left|\left(z_{1}+z_{2}\right) \phi\left(-\log \left|z_{1}+z_{2}\right|\right)-z_{1} \phi\left(-\log \left|z_{1}\right|\right)-z_{2} \phi\left(-\log \left|z_{2}\right|\right)\right| \\
= & \mid x_{1} \phi\left(-\log \left|z_{1}\right|\right)-x_{1} \phi\left(-\log \left|x_{1}\right|\right)+x_{1} \phi\left(-\log \left|x_{1}\right|\right) \\
+ & x_{2} \phi\left(-\log \left|z_{2}\right|\right)-x_{2} \phi\left(-\log \left|x_{2}\right|\right)+x_{2} \phi\left(-\log \left|x_{2}\right|\right) \\
- & \left(x_{1}+x_{2}\right) \phi\left(-\log \left|z_{1}+z_{2}\right|\right)+\left(x_{1}+x_{2}\right) \phi\left(-\log \left|x_{1}+x_{2}\right|\right)-\left(x_{1}+x_{2}\right) \phi\left(-\log \left|x_{1}+x_{2}\right|\right) \\
+ & i y_{1} \phi\left(-\log \left|z_{1}\right|\right)-i y_{1} \phi\left(-\log \left|y_{1}\right|\right)+i y_{1} \phi\left(-\log \left|y_{1}\right|\right) \\
+ & i y_{2} \phi\left(-\log \left|z_{2}\right|\right)-i y_{2} \phi\left(-\log \left|y_{2}\right|\right)+i y_{2} \phi\left(-\log \left|y_{2}\right|\right) \\
- & i\left(y_{1}+y_{2}\right) \phi\left(-\log \left|z_{1}+z_{2}\right|\right)+i\left(y_{1}+y_{2}\right) \phi\left(-\log \left|y_{1}+y_{2}\right|\right)-i\left(y_{1}+y_{2}\right) \phi\left(-\log \left|y_{1}+y_{2}\right|\right) \mid
\end{aligned}
$$




$$
\begin{gathered}
\leq\left|x_{1}\left(\phi\left(-\log \left|z_{1}\right|\right)-\phi\left(-\log \left|x_{1}\right|\right)\right)\right|+\left|x_{2}\left(\phi\left(-\log \left|z_{2}\right|\right)-\phi\left(-\log \left|x_{2}\right|\right)\right)\right| \\
+\left|\left(x_{1}+x_{2}\right)\left(\phi\left(-\log \left|x_{1}+x_{2}\right|\right)-\phi\left(-\log \left|z_{1}+z_{2}\right|\right)\right)\right|+L \log 2\left(\left|x_{1}\right|+\left|x_{2}\right|\right) \\
+\left|y_{1}\left(\phi\left(-\log \left|z_{1}\right|\right)-\phi\left(-\log \left|y_{1}\right|\right)\right)\right|+\left|y_{2}\left(\phi\left(-\log \left|z_{2}\right|\right)-\phi\left(-\log \left|y_{2}\right|\right)\right)\right| \\
+\left|\left(y_{1}+y_{2}\right)\left(\phi\left(-\log \left|y_{1}+y_{2}\right|\right)-\phi\left(-\log \left|z_{1}+z_{2}\right|\right)\right)\right|+L \log 2\left(\left|y_{1}\right|+\left|y_{2}\right|\right) \\
\leq L\left(\left|x_{1}\right| \log \left(\frac{\left|z_{1}\right|}{\left|x_{1}\right|}\right)+\left|x_{2}\right| \log \left(\frac{\left|z_{2}\right|}{\left|x_{2}\right|}\right)+\left|x_{1}+x_{2}\right| \log \left(\frac{\left|z_{1}+z_{2}\right|}{\left|x_{1}+x_{2}\right|}\right)+\log 2\left(\left|x_{1}\right|+\left|x_{2}\right|\right)\right. \\
\left.+\left|y_{1}\right| \log \left(\frac{\left|z_{1}\right|}{\left|y_{1}\right|}\right)+\left|y_{2}\right| \log \left(\frac{\left|z_{2}\right|}{\left|y_{2}\right|}\right)+\left|y_{1}+y_{2}\right| \log \left(\frac{\left|z_{1}+z_{2}\right|}{\left|y_{1}+y_{2}\right|}\right)+\log 2\left(\left|y_{1}\right|+\left|y_{2}\right|\right)\right) \\
\leq 2 L\left(\left|z_{1}\right|+\left|z_{2}\right|+\left|z_{1}+z_{2}\right|+\log 2\left(\left|z_{1}\right|+\left|z_{2}\right|\right)\right) \\
\leq 2 L(2+\log 2)\left(\left|z_{1}\right|+\left|z_{2}\right|\right) \\
\leq 8 L\left(\left|z_{1}\right|+\left|z_{2}\right|\right) .
\end{gathered}
$$

Denotemos por $\mathcal{L}$ a colecção de todas as funções de Lipschitz $\phi: \mathbb{R} \rightarrow \mathbb{K}$ tais que $\phi(t)=0$ para $t \leq 0$. Vamos usar os resultados obtidos até agora para construir operadores quase-lineares sobre espaços de sequências.

Teorema 2.34. Considere E um espaço quase-normado de sequências sobre um corpo $\mathbb{K}$ que satisfaz as seguintes propriedades

(a) Se $e_{n}$ denota o n-ésimo vetor da base canônica $\left(e_{n}(k)=\delta_{n k}\right)$, então o espaço linear $c_{00}(\mathbb{K})$ gerado por os vetores $\left(e_{n}\right)$ é denso em E.

(b) A quase norma $\|$.$\| em E satisfaz$

- $\|s x\| \leq\|s\|_{\infty}\|x\|$, para todo $s \in \ell_{\infty}$ e $x \in E$;

- $\left\|e_{n}\right\|=1$, para todo $n \in \mathbb{N}$;

- $\|x\|_{\infty} \leq\|x\|$, para todo $x \in X$.

Então, para cada $\phi \in \mathcal{L}$, existe um operador quase-linear $F_{\phi}: E \rightarrow E$, de modo que para cada $x \in c_{00}(\mathbb{K})$ se $x(k) \neq 0$, temos

$$
\begin{aligned}
F_{\phi}(x)[k] & =\|x\| \frac{x(k)}{\|x\|} \phi\left(-\log \frac{|x(k)|}{\|x\|}\right) \\
& =x(k) \phi\left(\log \frac{\|x\|}{|x(k)|}\right)
\end{aligned}
$$

Demonstração. Como consequência das propriedades (a) e (b) de $E$, temos que se $x \in E$ e $|x|$ denota a sequência cujo $k$-ésimo termo é igual a $|x(k)|$, então $|x| \in E$ e $\||x|\|=\|x\|$. Isto 
porque neste caso $\left(e_{n}\right)$ forma uma base incondicional de $E$ e tomando a sequência $s=(s(k))_{k}$ tal que para todo $k \in \mathbb{N}$, com $x(k) \neq 0$

$$
x(k) s(k)=|x(k)|
$$

e $s(k)=0$ se $x(k)=0$. Assim $\|s\|_{\infty} \leq 1 \mathrm{e}$

$$
\||x|\|=\|s x\| \leq\|s\|_{\infty}\|x\|=\|x\|=\left\|s^{-1}|x|\right\| \leq\left\|s^{-1}\right\|_{\infty}\||x|\| \leq\||x|\|
$$

onde $s^{-1}(k)=(s(k))^{-1}$ se $s(k) \neq 0$ e $s^{-1}(k)=0$ se $s(k)=0$. Do mesmo modo, observamos que se $x, y \in E$ são tais que $|x(k)| \leq|y(k)|$ par todo $k \in \mathbb{N}$, então $\|x\| \leq\|y\|$.

Seja $\phi \in \mathcal{L}$ definamos uma aplicação $f_{\phi}: c_{00}(\mathbb{K}) \rightarrow c_{00}(\mathbb{K})$ mediante a fórmula

$$
f_{\phi}(x)[k]=\left\{\begin{array}{rlll}
x(k) \phi(-\log |x(k)|), & \text { se } & x(k) \neq 0, & k \in \mathbb{N} \\
0, & \text { se } & x(k)=0, & k \in \mathbb{N}
\end{array}\right.
$$

$f_{\phi}$ assim definida é quase-aditiva(em relação à quase-norma de $E$ ). Para provar isto, observemos que pelo Lema (2.33) se $L$ é a constante de Lipschitz de $\phi$ e $x_{1}, x_{2} \in E$

$$
\left|f_{\phi}\left(x_{1}\right)[k]+f_{\phi}\left(x_{2}\right)[k]-f_{\phi}\left(x_{1}+x_{2}\right)[k]\right| \leq 8 L\left(\left|x_{1}(k)\right|+\left|x_{2}(k)\right|\right) .
$$

Segue do comentário anterior que

$$
\begin{aligned}
\left\|f_{\phi}\left(x_{1}\right)+f_{\phi}\left(x_{2}\right)-f_{\phi}\left(x_{1}+x_{2}\right)\right\| & \leq 8 L\left\|\left|x_{1}\right|+\left|x_{2}\right|\right\| \\
& \leq 8 L K\left(\left\|x_{1}\right\|+\left\|x_{2}\right\|\right)
\end{aligned}
$$

Onde $K$ é o módulo de concavidade de $\|$.$\| . Agora pelo Teorema (2.32) f_{\phi}$ induz uma aplicação quase-linear $F_{0}: c_{00}(\mathbb{K}) \rightarrow c_{00}(\mathbb{K})$ que podemos extender(Teorema (2.28)) a um operador quase-linear $F_{\phi}: E \rightarrow E$. E, pela construção $F_{\phi}$ satisfaz a fórmula do enunciado.

O seguinte teorema é também um método de construção de somas torcidas, agora em espaços mais gerais do que o teorema anterior.

Teorema 2.35. Sejam $X$ e $Y$ espaços quase-normados e $Z$ um espaço vetorial tal que $Y \subseteq Z$. Suponhamos que exista $\Omega: X \rightarrow Z$ uma aplicação tal que

(a) $\Omega(\lambda x)=\lambda \Omega(x)$ para todo $x \in X$ e $\lambda \in \mathbb{K}$.

(b) $\Omega\left(x_{1}+x_{2}\right)-\Omega\left(x_{1}\right)-\Omega\left(x_{2}\right) \in Y$ para todo par $x_{1}, x_{2} \in X$. Além disso, existe uma constante $K>0$ tal que

$$
\left\|\Omega\left(x_{1}+x_{2}\right)-\Omega\left(x_{1}\right)-\Omega\left(x_{2}\right)\right\|_{Y} \leq K\left(\left\|x_{1}\right\|_{X}+\left\|x_{2}\right\|_{X}\right) .
$$


Se denotamos por $E=\{(x, z) \in X \times Z ; \quad z-\Omega(x) \in Y\}$ e definimos para $(x, z) \in E$

$$
\|(x, z)\|_{\Omega}=\|x\|_{X}+\|z-\Omega(x)\|_{Y} .
$$

Então $\left(E,\|.\|_{\Omega}\right)$ é um espaço quase-normado isométrico a uma soma torcida $Y \oplus_{\Omega^{\prime}} X$, para algum operador quase-linear $\Omega^{\prime}: X \rightarrow Y$, tal que $\Omega-\Omega^{\prime}: X \rightarrow Y$ é linear.

Demonstração. Pelas propriedades de $\Omega$ é fácil notar que $E$ é um espaço vetorial. Vejamos que $\|.\|_{\Omega}$ é uma quase-norma em $E$. Considere $M$ um módulo de concavidade para as quasenormas em $X$ e $Y$ e tomemos $\left(x_{i}, z_{i}\right) \in E, i=1,2$.

$$
\begin{aligned}
\left\|\left(x_{1}, z_{1}\right)+\left(x_{2}, z_{2}\right)\right\|_{\Omega} & =\left\|x_{1}+x_{2}\right\|_{X}+\left\|z_{1}+z_{2}-\Omega\left(x_{1}+x_{2}\right)\right\|_{Y} \\
& \leq M\left(\left\|x_{1}\right\|_{X}+\left\|x_{2}\right\|_{X}+\left\|z_{1}-\Omega\left(x_{1}\right)\right\|_{Y}\right) \\
& +M^{2}\left(\left\|z_{2}-\Omega\left(x_{2}\right)\right\|_{Y}+\left\|\Omega\left(x_{1}+x_{2}\right)-\Omega\left(x_{1}\right)-\Omega\left(x_{2}\right)\right\|_{Y}\right) \\
& \leq\left(M+M^{2} K\right)\left(\left\|\left(x_{1}, z_{1}\right)\right\|_{\Omega}+\left\|\left(x_{2}, z_{2}\right)\right\|_{\Omega}\right)
\end{aligned}
$$

Seja $Q: Z \rightarrow Z / Y$ a aplicação quociente. Pelo Lema (1.6) existe uma aplicação linear $\eta: Z / Y \rightarrow Z$ tal que $Q \circ \eta=i d_{Z / Y}$. Dado que $Q \circ \Omega: X \rightarrow Z / Y$ é linear( condição (b) de $\Omega)$, definamos $A=\eta(Q \circ \Omega)$. Portanto $Q \circ A=Q \circ \Omega$ e em consequência $\Omega-A(x) \in Y$ para todo $x \in X$. Escrevamos $\Omega^{\prime}=\Omega-A: X \rightarrow Y$. Verificamos pelas propriedades (a) e (b) que $\Omega^{\prime}$ é um operador quase-linear, pois

$$
\Omega^{\prime}\left(x_{1}+x_{2}\right)-\Omega^{\prime}\left(x_{1}\right)-\Omega^{\prime}\left(x_{2}\right)=\Omega\left(x_{1}+x_{2}\right)-\Omega\left(x_{1}\right)-\Omega\left(x_{2}\right)
$$

para todo $x_{1}, x_{2} \in X$. Formemos a soma torcida $Y \oplus_{\Omega^{\prime}} X$ com a quase-norma

$$
\|(y, x)\|_{\Omega^{\prime}}=\|x\|_{X}+\left\|y-\Omega^{\prime}(x)\right\|_{Y}
$$

e definamos $T: Y \oplus_{\Omega^{\prime}} X \rightarrow E$ mediante a fórmula $T(y, x)=(x, y+A x)$. Com isto, $T$ está bem definida, pois $y+A x-\Omega(x)=y-\Omega^{\prime}(x) \in Y$. Além disso

$$
\begin{aligned}
\|T(x, y)\|_{\Omega} & =\|x\|_{X}+\|y+A x-\Omega(x)\|_{Y} \\
& =\|x\|_{2}+\left\|y-\Omega^{\prime}(x)\right\|_{Y} \\
& =\|(x, y)\|_{\Omega^{\prime}}
\end{aligned}
$$

Por último, $T$ é sobrejetora, já que $(x, z)=T(z-A x, x)$ e para $(x, z) \in E$ temos $z-A x=$ $(z-\Omega(x))+(\Omega(x)-A x) \in Y$. Logo $T$ é uma isometría linear entre $E$ e $Y \oplus_{\Omega^{\prime}} X$. 


\section{Capítulo 3}

\section{Exemplo de um espaço de Banach não isomorfo a seu conjugado complexo}

Neste capítulo, para cada $\alpha \in(-\infty, \infty)$ vamos definir um espaço de Banach $Z_{2}(\alpha)$ que é uma soma torcida de $\ell_{2}$ com $\ell_{2}$. Demonstraremos que o espaço conjugado de $Z_{2}(\alpha)$ é isomorfo a $Z_{2}(-\alpha)$. Usando o fato que cada $Z_{2}(\alpha)$ possui uma F.D.D contrátil, mostraremos que se $\alpha \neq \beta$ então $Z_{2}(\alpha)$ não é isomorfo com nenhum subespaço de $Z_{2}(\beta)$. Portanto, para $\alpha \neq 0$, $Z_{2}(\alpha)$ não é isomorfo ao conjugado.

\subsection{Construção do espaço $Z_{2}(\alpha)$}

Usando as técnicas do capítulo anterior, vamos construir um espaço de Banach não isomorfo a seu conjugado complexo.

Com a notação do Teorema (2.35), seja $Z=\mathbb{C}^{\mathbb{N}}$ o conjunto de todas as sequências de valores complexos e $X=Y=\ell_{2}$ subespaço de $Z$. Para cada função de Lipschitz $\phi:[0, \infty] \rightarrow \mathbb{C}$, definamos uma aplicação $\Omega_{\phi}: \ell_{2} \rightarrow \mathbb{C}^{\mathbb{N}}$ mediante

$$
\Omega_{\phi}(x)(n)= \begin{cases}x(n) \phi\left(\log \frac{\|x\|_{2}}{|x(n)|}\right) & \text { se } x(n) \neq 0 \\ 0 & \text { se } x(n)=0\end{cases}
$$

É claro que $\Omega_{\phi}(\lambda x)=\lambda \Omega_{\phi}(x)$ par todo $\lambda \in \mathbb{C}$.

Escrevamos $f_{\phi}$ como na fórmula (2.8) da seção anterior

$$
f_{\phi}(x)(n)=\left\{\begin{array}{rlll}
x(n) \phi(-\log |x(n)|), & \text { se } & x(n) \neq 0, & k \in \mathbb{N} \\
0, & \text { se } & x(n)=0, & k \in \mathbb{N}
\end{array}\right.
$$

Segue do Lema (2.33) que se $L$ é a constante de Lipschitz de $\phi$, então

$$
\left\|f_{\phi}\left(x_{1}\right)+f_{\phi}\left(x_{2}\right)-f_{\phi}\left(x_{1}+x_{2}\right)\right\|_{2} \leq 8 L\left(\left\|x_{1}\right\|_{2}+\left\|x_{2}\right\|_{2}\right) \text {. }
$$


Também observemos que para cada $x \in \ell_{2} \operatorname{com}\|x\|_{2} \leq 1$ e cada $x(n) \neq 0$

$$
\begin{aligned}
\left|\Omega_{\phi}(x)(n)-f_{\phi}(x)(n)\right| & =\left|x(n) \phi\left(\log \frac{\|x\|_{2}}{|x(n)|}\right)-x(n) \phi(-\log |x(n)|)\right| \\
& \leq L|x(n)|\left|\log \left(\frac{\|x\|_{2}|x(n)|}{|x(n)|}\right)\right| \\
& \leq L \frac{|x(n)|}{\|x\|_{2}}
\end{aligned}
$$

Em consequência $\left\|\Omega_{\phi}(x)-f_{\phi}(x)\right\|_{2} \leq L$. Suponhamos $\left\|x_{1}\right\|_{2}+\left\|x_{2}\right\|_{2} \leq 1$

$$
\begin{gathered}
\left\|\Omega_{\phi}\left(x_{1}+x_{2}\right)-\Omega_{\phi}\left(x_{1}\right)-\Omega_{\phi}\left(x_{2}\right)\right\| \leq\left\|\Omega_{\phi}\left(x_{1}+x_{2}\right)-f_{\phi}\left(x_{1}+x_{2}\right)\right\|_{2} \\
+\left\|\Omega_{\phi}\left(x_{1}\right)-f_{\phi}\left(x_{2}\right)\right\|_{2}+\left\|\Omega_{\phi}\left(x_{2}\right)-f_{\phi}\left(x_{2}\right)\right\|_{2}+ \\
\left\|f_{\phi}\left(x_{1}\right)+f_{\phi}\left(x_{2}\right)-f_{\phi}\left(x_{1}+x_{2}\right)\right\| \leq 11 L
\end{gathered}
$$

Agora, se $x_{1}, x_{2} \in \ell_{2}$ arbitrários, fica claro que

$$
\left\|\Omega_{\phi}\left(x_{1}+x_{2}\right)-\Omega_{\phi}\left(x_{1}\right)-\Omega_{\phi}\left(x_{2}\right)\right\|_{2} \leq 11 L\left(\left\|x_{1}\right\|_{2}+\left\|x_{2}\right\|_{2}\right)
$$

Portanto, $\Omega_{\phi}$ satisfaz as condições (a) e (b) do Teorema (2.35). Denotemos por $Z_{2}(\phi)=$ $\left\{(x, y) \in \ell_{2} \times \mathbb{C}^{\infty} ; \quad y-\Omega_{\phi}(x) \in \ell_{2}\right\}$. Então

$$
\|(x, y)\|_{\phi}=\|x\|_{2}+\left\|y-\Omega_{\phi}(x)\right\|_{2}
$$

define uma quase-norma em $Z_{2}(\phi)$. Assim, podemos considerar $\left(Z_{2}(\phi),\|\cdot\|_{\phi}\right)$ como uma soma torcida de $\ell_{2}$ com ele mesmo e em consequência $Z_{2}(\phi)$ será um espaço de Banach reflexivo(Teoremas $(2.27)$ e $(2.15))$ com uma norma equivalente a $\|\cdot\|_{\phi}$.

Em particular vamos estudar os espaços $Z_{2}(\phi)$ para as funções $\phi_{\alpha}(t)=t^{1+i \alpha}, t \geq 0$ para $\alpha \in(-\infty, \infty)$. Denotamos por $Z_{2}(\alpha)$ o espaço $Z_{2}\left(\phi_{\alpha}\right)$ e $\Omega_{\alpha}$ no lugar de $\Omega_{\phi_{\alpha}}$

\subsection{Propriedades do espaço $Z_{2}(\alpha)$}

Lema 3.1. A sequência de subespaços $\left\{E_{n}\right\}_{n=1}^{\infty}$ de $Z_{2}(\phi)$, onde $E_{n}=\operatorname{span}\left\{\left(e_{n}, 0\right),\left(0, e_{n}\right)\right\}$, é uma 2-F.D.D contrátil de $Z_{2}(\phi)$.

Demonstração. Seja $(x, y) \in Z_{2}(\phi)$. Vamos mostrar que $\sum_{n=1}^{\infty}\left(x(n) e_{n}, y(n) e_{n}\right)$ converge a 
$(x, y)$ em $Z_{2}(\phi)$. Dado $n \in \mathbb{N}$ e $z \in \ell_{2}$, denotaremos por $z^{n}:=\sum_{k>n} z(k) e_{k}$. Assim

$$
\begin{aligned}
\left\|(x, y)-\sum_{k=1}^{n}\left(x(k) e_{k}, y(k) e_{k}\right)\right\|_{\phi} & =\left\|x^{n}\right\|_{2}+\left\|\sum_{k>n}\left(y(k)-x(k) \phi\left(\log \frac{\left\|x^{n}\right\|_{2}}{|x(k)|}\right)\right) e_{k}\right\|_{2} \\
& \leq\left\|x^{n}\right\|_{2}+\left\|\left(y-\Omega_{\phi}(x)\right)^{n}\right\|_{2}+L\left\|\sum_{k>n} x(k) \log \left(\frac{\|x\|_{2}}{\left\|x^{n}\right\|_{2}}\right)\right\|_{2} \\
& =\left\|x^{n}\right\|_{2}+\left\|\left(y-\Omega_{\phi}(x)\right)^{n}\right\|_{2}+L\left\|x^{n}\right\|_{2} \log \left(\frac{\|x\|_{2}}{\left\|x^{n}\right\|_{2}}\right)
\end{aligned}
$$

e a última expressão tem limite zero quando $n \rightarrow \infty$. Portanto, a série $\sum_{n=1}^{\infty}\left(x(n) e_{n}, y(n) e_{n}\right)$ converge para $(x, y)$. Dado que $\operatorname{dim} E_{n}=2$, concluímos que $\left\{E_{n}\right\}_{n=1}^{\infty}$ é uma 2-F.D.D. Segue do Teorema (1.52) que a decomposição é contrátil, pois $Z_{2}(\phi)$ é um espaço reflexivo.

Observação 3.2. Dada uma sequência crescente $k=\left(k_{n}\right)_{n=1}^{\infty}$ de inteiros positivos, o operador $T_{k}: Z_{2}(\phi) \rightarrow Z_{2}(\phi)$ definido por $T_{k}(x, y)=\left(x_{\pi_{k}}, y_{\pi_{k}}\right)$ onde $x_{\pi_{k}}(n)=x\left(k_{n}\right)$, é linear e contínuo, pois $\left\|T_{k}(x, y)\right\|_{\phi} \leq\|(x, y)\|_{\phi}$, assim $\left\|T_{k}\right\| \leq 1$. Nos próximos argumentos, vamos estudar o comportamento de certos operadores definidos em $Z_{2}(\phi)$. Usando $T_{k}$ será possível simplificar a análise destes, considerando só o seu comportamento nos subespaços $E_{k_{n}}$ relativos à sequência $k$.

Lema 3.3. Seja $\beta \in(-\infty, \infty)$. Se $b=1+i \beta$, então para $t>s>0$

$$
\begin{aligned}
\left|t^{b}-s^{b}\right| & \geq t-s \\
\left|t^{b}-s^{b}\right| & \leq|b|(t-s) \\
\left|t^{b}-s^{b}-b s^{b-1}(t-s)\right| & \leq \frac{1}{2}|b||s|^{-1}(t-s)^{2}
\end{aligned}
$$

Demonstração. A primeira desigualdade resulta de $\left|t^{b}-s^{b}\right| \geq\left|t^{b}\right|-\left|s^{b}\right|=t-s$. Para obter (3.2), derivamos $\phi_{\beta}^{\prime}(t)=(1+i \beta) t^{1+i \beta} t^{-1}=b t^{i \beta}$, logo $\left|\phi_{\beta}^{\prime}(t)\right| \leq|\beta|$ para todo $t>0$, assim $\left|t^{b}-s^{b}\right| \leq|b|(t-s)$. Para a terceira fórmula, usamos aproximação por Taylor, ou seja, para algum $s<u<t$ temos $\left|\phi_{\beta}(t)-\phi_{\beta}(s)-\phi_{\beta}^{\prime}(s)(t-s)\right|=\left|\frac{1}{2} \phi_{\beta}^{\prime \prime}(u)\right|(t-s)^{2}=$ $\frac{1}{2}\left|b u^{i \beta} u^{-1}\right|(t-s)^{2} \leq \frac{1}{2}|b||s|^{-1}(t-s)^{2}$.

Dado $\beta \in(-\infty, \infty)$, escrevamos $b=1+i \beta$. Definamos para cada $w \in \ell_{2}$ uma sequência $\Omega_{\beta}^{\prime}(w)$, mediante a fórmula

$$
\Omega_{\beta}^{\prime}(w)(n)=\left\{\begin{array}{rll}
w(n)\left(\log |w(n)|^{-1}\right)^{b}, & \text { se } & w(n) \neq 0 \\
0, & \text { se } & w(n)=0
\end{array}\right.
$$

Pela definição $\Omega_{\beta}^{\prime}(w)$ preserva o suporte da sequência $w$, no sentido que $\Omega_{\beta}^{\prime}(w)(n)=0$ para 
todo $n$ tal que $w(n)=0$. Também observamos que

$$
\begin{aligned}
\left|\Omega_{\beta}(w)(n)-\Omega_{\beta}^{\prime}(w)(n)\right| & =\left|w(n) f_{\beta}\left(\log \frac{\|w\|_{2}}{|w(n)|}\right)-w(n) f_{\beta}\left(\log \frac{1}{|w(n)|}\right)\right| \\
& \leq|w(n)||b|\left|\log \frac{\|w\|_{2}}{|w(n)|}-\log \frac{1}{|w(n)|}\right| \\
& =|w(n)||b|\left|\log \|w\|_{2}\right|
\end{aligned}
$$

Para $\|w\|_{2} \leq 1$ temos $\left|\log \|w\|_{2}\right| \leq 1 /\|w\|_{2}$ e portanto

$$
\left\|\Omega_{\beta}(w)-\Omega_{\beta}^{\prime}(w)\right\|_{2} \leq|b|
$$

Se $A$ é um subconjunto finito de $\mathbb{N}$, vamos denotar por $\xi_{A}=\sum_{n \in A} e_{n}$. Para $u \in \mathbb{C}^{\mathbb{N}}$, escreveremos $u \xi_{A}=\sum_{n \in A} u(n) e_{n}$.

Lema 3.4. O conjugado complexo de $Z_{2}(\alpha)$ é isomorfo a $Z_{2}(-\alpha)$

Demonstração. Seja $\alpha \in(-\infty, \infty)$. Definamos o operador $T: Z_{2}(-\alpha) \rightarrow \overline{Z_{2}(\alpha)}$ por

$$
T(x, y)=(\bar{x}, \bar{y})
$$

onde $\bar{x}$ representa a sequência $\bar{x}(k)=\overline{x(k)}$ para cada $k \in \mathbb{N}$. Do fato que $\overline{t^{1+i \alpha}}=t^{1-i \alpha}, t \geq 0$ temos

$$
\bar{y}(n)-\bar{x}(n) f_{\alpha}\left(\log \frac{\|\bar{x}\|_{2}}{|\bar{x}(n)|}\right)=\overline{y(n)-x(n) f_{-\alpha}\left(\log \frac{\|x\|_{2}}{|x(n)|}\right.}
$$

segue-se que $\|T(x, y)\|_{\alpha}=\|(x, y)\|_{-\alpha}, \operatorname{logo} T$ está bem definido e é bijetor. $\mathrm{T}$ é $\mathbb{C}$ linear, pois $T(\lambda(x, y))=\lambda \odot T(x, y)$. Assim, $T$ é um isomorfismo $\mathbb{C}$ linear entre os espaços $Z_{2}(-\alpha)$ e $\overline{Z_{2}(\alpha)}$.

Definição 3.5. Um operador linear contínuo $T: Z_{2}(\alpha) \rightarrow Z_{2}(\beta)$ será chamado c-admissível, para algum $c>0$, se $\|T\|<1$ e $\left\|T\left(e_{n}, 0\right)\right\|_{\beta},\left\|T\left(0, e_{n}\right)\right\|_{\beta}>$ c para todo $n \in \mathbb{N}$.

\subsection{Isomorfismos entre os espaços $Z_{2}(\alpha)$}

O objetivo principal é provar o seguinte teorema.

Teorema 3.6. Se $Z_{2}(\alpha)$ é isomorfo a um subespaço de $Z_{2}(\beta)$, então $\alpha=\beta$.

Seja $\alpha \neq 0$ e suponhamos que $Z_{2}(\alpha)$ seja isomorfo a um subespaço de $Z_{2}(\beta)$. A prova do teorema resulta das seguintes afirmações:

Afirmação 1. Existe um operador linear contínuo $T: Z_{2}(\alpha) \rightarrow Z_{2}(\beta) c$-admissível. E existem sequências complexas $u, v, w$ e $y$ e uma sequência crescente de inteiros positivos 
$\left(p_{n}\right)_{n=0}^{\infty}$ tais que

$$
\begin{aligned}
& T\left(e_{n}, 0\right)=\left(u \xi_{B_{n}}, v \xi_{B_{n}}\right) \\
& T\left(0, e_{n}\right)=\left(w \xi_{B_{n}}, y \xi_{B_{n}}\right)
\end{aligned}
$$

onde $B_{n}=\left\{p_{n-1}, \ldots, p_{n}-1\right\}$.

Afirmação 2. O operador $T$ obtido na afirmação 1 satisfaz $\lim _{n \rightarrow \infty}\left\|w \xi_{B_{n}}\right\|_{2}=0$.

Afirmação 3. Existe um operador linear contínuo $T: Z_{2}(\alpha) \rightarrow Z_{2}(\beta)$-admissível. E existem sequências complexas $u, v$ e $y$ e uma sequência crescente de inteiros positivos $\left(p_{n}\right)_{n=0}^{\infty}$ tais que

$$
\begin{aligned}
& T\left(e_{n}, 0\right)=\left(u \xi_{B_{n}}, v \xi_{B_{n}}\right) \\
& T\left(0, e_{n}\right)=\left(0 \xi_{B_{n}}, y \xi_{B_{n}}\right)
\end{aligned}
$$

onde $B_{n}=\left\{p_{n-1}, \ldots, p_{n}-1\right\}$. Em outras palavras podemos tomar o operador $T$ da afirmação 1 , tal que $w=0$.

Afirmação 4. O operador $T$ obtido na afirmação 3 satisfaz $\inf _{n \in \mathbb{N}}\left\|u \xi_{B_{n}}\right\|_{\infty} \neq 0$.

Afirmação 5. Existe um operador linear contínuo $S: Z_{2}(\alpha) \rightarrow Z_{2}(\beta)$ com $\|S\|<1$ tal que

$$
\begin{aligned}
& S\left(e_{n}, 0\right)=\left(\lambda e_{n}, \mu e_{n}\right) \\
& S\left(0, e_{n}\right)=\left(0, \nu e_{n}\right)
\end{aligned}
$$

para constantes $\lambda, \mu$ e $\nu$ com $\lambda \neq 0$.

Afirmação 6. A existência de um operador como o obtido na afirmação 5 implica que $\alpha=\beta$.

Escrevamos $a=1+i \alpha$ e $b=1+i \beta$.

Afirmação 1. Vamos a obter um operador $T: Z_{2}(\alpha) \rightarrow Z_{2}(\beta) c$-admissível, para algum $c>0$, e uma sequência crescente de inteiros positivos $\left(p_{n}\right)_{n=0}^{\infty}$ tal que $T\left(e_{n}, 0\right)=$ $\left(u \xi_{B_{n}}, v \xi_{B_{n}}\right)$ e $T\left(0, e_{n}\right)=\left(w \xi_{B_{n}}, y \xi_{B_{n}}\right)$ para certas sequências complexas $u, v, w$ e $y$, onde $B_{n}=\left\{p_{n-1}, \ldots, p_{n}-1\right\}$.

Considerando um múltiplo escalar de um operador que determina o isomorfismo entre $Z_{2}(\alpha)$ e um subespaço de $Z_{2}(\beta)$ obtemos um operador linear contínuo $T: Z_{2}(\alpha) \rightarrow Z_{2}(\beta) c$ admissível. Pelo Lema (3.1) os subespaços $\left\{E_{n}\right\}_{n=1}^{\infty}$ formam uma 2-F.D.D contrátil de $Z_{2}(\alpha)$. Sejam $\epsilon>0$, tal que $\|T\|+\epsilon<1$ e $\left(\epsilon_{n}\right)_{n}$ uma sequência de números reais positivos tais que

$$
\begin{gathered}
\left\|T\left(e_{n}, 0\right)\right\|_{\beta}-\epsilon_{n}>c \\
\left\|T\left(0, e_{n}\right)\right\|_{\beta}-\epsilon_{n}>c
\end{gathered}
$$


e $\sum_{n} \epsilon_{n}<\epsilon$. Aplicando o Teorema (1.54) e seu corolario (1.55), obtemos um operador linear contínuo $\widetilde{T}: Z_{2}(\alpha) \rightarrow Z_{2}(\beta)$ e 'blockings' $\left\{F_{n}\right\}_{n=0}^{\infty}$ de $\left\{E_{n}\right\}$ em $Z_{2}(\alpha)$ e $\left\{G_{n}\right\}_{n=0}^{\infty}$ de $\left\{E_{n}\right\}$ em $Z_{2}(\beta)$, satisfazendo

(a) $\widetilde{T}(x) \in G_{n}$, para todo $x \in F_{2 n}$.

(b) $\|\widetilde{T}(x)\|_{\beta} \geq\|T x\|_{\beta}-\epsilon_{n}\|x\|_{\alpha}$, para todo $x \in F_{n}$.

(c) $\|\widetilde{T}\| \leq\|T\|+\epsilon<1$.

Supondo que os 'blockings' $\left\{F_{n}\right\}_{n=0}^{\infty}$ e $\left\{G_{n}\right\}_{n=0}^{\infty}$ são gerados pelas sequências $t_{0}=1<t_{1}<$ $t_{2}<\ldots$ e $p_{0}=1<p_{1}<p_{2}<\ldots$, respetivamente, definamos $k=\left(k_{n}\right)_{n}$, uma sequência, onde $k_{n}=t_{2(n-1)}$ para $n \geq 1$. Usando o operador $T_{k}$ (definido na observação (3.2)) associado à sequência $k$ e escrevendo $S=\widetilde{T} \circ T_{k}$, obtemos

$$
\begin{gathered}
S\left(e_{n}, 0\right)=\left(u \xi_{B_{n}}, v \xi_{B_{n}}\right) \\
S\left(0, e_{n}\right)=\left(w \xi_{B_{n}}, y \xi_{B_{n}}\right)
\end{gathered}
$$

onde $u, v, w$ e $y$ são sequências complexas e $B_{n}=\left\{p_{n-1}, \ldots, p_{n}-1\right\}$ para $n \geq 1$. Além disso

$$
\left\|S\left(e_{n}, 0\right)\right\|_{\beta}=\left\|\widetilde{T}\left(e_{t_{2(n-1)}}, 0\right)\right\|_{\beta} \geq\left\|T\left(e_{t_{2(n-1)}}, 0\right)\right\|_{\beta}-\epsilon_{t_{2(n-1)}}>c .
$$

De modo análogo, $\left\|S\left(0, e_{n}\right)\right\|_{\beta}>c$ para todo $n \in \mathbb{N}$. Notemos também que $\|S\| \leq\|\widetilde{T}\|<1$. Logo $S$ é o operador requerido.

Afirmação 2. Vamos provar para o operador obtido na afirmação anterior que $\lim _{n \rightarrow \infty}\left\|w \xi_{B_{n}}\right\|_{2}=$ 0 .

Caso contrário, existiria um $\delta>0$ e uma sequência crescente $\left(k_{n}\right)_{n=1}^{\infty}$ de inteiros positivos tais que $\left\|w \xi_{B_{k_{n}}}\right\|_{2} \geq \delta$ para todo $n$. Pela observação (3.2), podemos supor que $k_{n}=n$ para todo $n \in \mathbb{N}$, pois é simplesmente trabalhar com $T \circ T_{k}$.

Dado um inteiro $N$, seja $A=\{1,2, \ldots, N\}$. Notemos que

$$
\begin{aligned}
\left\|T\left(0, N^{-1 / 2} \xi_{A}\right)\right\|_{\beta} & \leq\|T\|\left\|\left(0, N^{-1 / 2} \xi_{A}\right)\right\|_{\alpha} \\
& =\|T\|<1
\end{aligned}
$$

pois $\left\|\left(0, N^{-1 / 2} \xi_{A}\right)\right\|_{\alpha}=N^{-1 / 2}\left\|\xi_{A}\right\|_{2}=1 . \operatorname{Logo}$ 


$$
\left\|N^{-1 / 2} \sum_{j \in A} y \xi_{B_{j}}-\Omega_{\beta}\left(N^{-1 / 2} \sum_{j \in A} w \xi_{B_{j}}\right)\right\| \leq 1
$$

já que $T\left(0, N^{-1 / 2} \xi_{A}\right)=N^{-1 / 2}\left(\sum_{j \in A} y \xi_{B_{j}}, \sum_{j \in A} w \xi_{B_{j}}\right)$.

De forma análoga

$$
\begin{aligned}
\left\|T\left(0, e_{j}\right)\right\|_{\beta} & \leq\|T\|\left\|\left(0, e_{j}\right)\right\|_{\alpha} \\
& =\|T\|<1
\end{aligned}
$$

e pela equação $(3.5)$

$$
\begin{aligned}
\left\|T\left(0, e_{j}\right)\right\|_{\beta} & =\left\|\left(w \xi_{B_{j}}, y \xi_{B_{j}}\right)\right\|_{\beta} \\
& =\left\|y \xi_{B_{j}}-\Omega_{\beta}\left(w \xi_{B_{j}}\right)\right\|_{2}+\left\|w \xi_{B_{j}}\right\|_{2} .
\end{aligned}
$$

Em consequência $\left\|w \xi_{B_{j}}\right\|_{2}<1$ para todo $j \in \mathbb{N}$. Agora, como $B_{i} \cap B_{j}=\emptyset$ para $i \neq j$, observamos que

$$
\left\|N^{-1 / 2} \sum_{j \in A} w \xi_{B_{j}}\right\|_{2} \leq 1
$$

Com efeito,

$$
\begin{aligned}
\left\|\sum_{j \in A} w \xi_{B_{j}}\right\|_{2}^{2} & =\sum_{j \in A} \sum_{k \in B_{j}}|w(k)|^{2} \\
& =\sum_{j \in A}\left\|w \xi_{B_{j}}\right\|^{2} \leq N .
\end{aligned}
$$

Usando o mesmo argumento, e o fato que $\left\|y \xi_{B_{j}}-\Omega_{\beta}\left(w \xi_{B_{j}}\right)\right\|_{2}<1$ segue também que

$$
\left\|N^{-1 / 2} \sum_{j \in A} y \xi_{B_{j}}-\sum_{j \in A} \Omega_{\beta}\left(N^{-1 / 2} w \xi_{B_{j}}\right)\right\|_{2} \leq 1
$$

pois $\Omega_{\beta}$ preserva o suporte das sequências. Por (3.6) , (3.8) e desigualdade triangular, obtemos

$$
\left\|\Omega_{\beta}\left(N^{-1 / 2} \sum_{j \in A} w \xi_{B_{j}}\right)-N^{-1 / 2} \sum_{j \in A} \Omega_{\beta}\left(w \xi_{B_{j}}\right)\right\|_{2} \leq 2
$$


Agora, por (3.7) e a equação (3.4)

$$
\left\|\Omega_{\beta}\left(N^{-1 / 2} \sum_{j \in A} w \xi_{B_{j}}\right)-\Omega_{\beta}^{\prime}\left(N^{-1 / 2} \sum_{j \in A} w \xi_{B_{j}}\right)\right\| \leq|b|
$$

e para todo $j$

$$
\left\|\Omega_{\beta}\left(w \xi_{B_{j}}\right)-\Omega_{\beta}^{\prime}\left(w \xi_{B_{j}}\right)\right\| \leq|b|
$$

Da equação anterior obtemos

$$
\left\|N^{-1 / 2} \sum_{j \in A} \Omega_{\beta}\left(w \xi_{B_{j}}\right)-N^{-1 / 2} \sum_{j \in A} \Omega_{\beta}^{\prime}\left(w \xi_{B_{j}}\right)\right\| \leq|b|
$$

Pois $\Omega_{\beta}^{\prime}$ também preserva o suporte. Novamente pela desigualdade triangular, juntando (3.9), (3.10) e (3.11)

$$
\left\|\Omega_{\beta}^{\prime}\left(N^{-1 / 2} \sum_{j \in A} w \xi_{B_{j}}\right)-N^{-1 / 2} \sum_{j \in A} \Omega_{\beta}^{\prime}\left(w \xi_{B_{j}}\right)\right\| \leq 2(1+|b|)
$$

Desenvolvendo a fórmula anterior, que só interessa quando $w(k) \neq 0$, obtemos

$$
\frac{1}{N} \sum_{j=1}^{N} \sum_{k \in B_{j}}|w(k)|^{2}\left|\left(\log \frac{\sqrt{N}}{|w(k)|}\right)^{b}-\left(\log \frac{1}{|w(k)|}\right)^{b}\right|^{2} \leq 4(1+|b|)^{2} .
$$

Segue do Lema (3.3) equação (3.1)

$$
\left|\left(\log \frac{\sqrt{N}}{|w(k)|}\right)^{b}-\left(\log \frac{1}{|w(k)|}\right)^{b}\right| \geq \log (\sqrt{N})
$$

e assim

$$
\sum_{k \in B_{j}}|w(k)|^{2}\left|\left(\log \frac{\sqrt{N}}{|w(k)|}\right)^{b}-\left(\log \frac{1}{|w(k)|}\right)^{b}\right|^{2} \geq\left\|w \xi_{B_{j}}\right\|_{2}^{2}(\log \sqrt{N})^{2}
$$

Portanto

$$
\begin{aligned}
4(1+|b|)^{2} & \geq \frac{1}{N} \sum_{j=1}^{N}\left\|w \xi_{B_{j}}\right\|_{2}^{2}(\log \sqrt{N})^{2} \\
& \geq \delta^{2}(\log \sqrt{N})^{2}
\end{aligned}
$$

para todo $N \in \mathbb{N}$. Isto é um absurdo, $\log \lim _{n \rightarrow \infty}\left\|w \xi_{B_{n}}\right\|_{2}=0$. 
Afirmação 3. Vamos observar que pelo fato anterior, existe um operador $c$ admissível com as propriedades da afirmação 1 , tal que $w=0$.

Definamos operadores $W_{n}: E_{n} \rightarrow Z_{2}(\beta)$ para cada $n$ da seguinte forma

$$
\begin{aligned}
& W_{n}\left(0, e_{n}\right)=\left(w \xi_{B_{n}}, \Omega_{\beta}\left(w \xi_{B_{n}}\right)\right) \\
& W_{n}\left(e_{n}, 0\right)=0
\end{aligned}
$$

e extendamos a $E_{n}$ por linearidade, ou seja $W_{n}\left(x_{n} e_{n}, y_{n} e_{n}\right)=y_{n} W_{n}\left(0, e_{n}\right)$. Observemos que

$$
\begin{aligned}
\left\|W_{n}\left(x_{n} e_{n}, y_{n} e_{n}\right)\right\|_{\beta} & \leq\left|y_{n}\right|\left\|\left(w \xi_{B_{n}}, \Omega_{\beta}\left(w \xi_{B_{n}}\right)\right)\right\|_{\beta} \\
& =\left|y_{n}\right|\left\|w \xi_{B_{n}}\right\|_{2} \\
& \leq\left\|w \xi_{B_{n}}\right\|_{2}\left\|\left(x_{n} e_{n}, y_{n} e_{n}\right)\right\|_{\alpha}
\end{aligned}
$$

Com isto, $\left\|W_{n}\right\| \leq\left\|w \xi_{B_{n}}\right\|_{2}$.

Tomemos $\epsilon>0$ de modo que $\|T\|+\epsilon<1$. Como na afirmação 1 , podemos obter uma sequência $\left(\epsilon_{n}\right)_{n}$ de números reais positivos tais que $\sum_{n=1}^{\infty} \epsilon_{n}<\epsilon / 2 \mathcal{K}$ onde $\mathcal{K}$ é a constante da decomposição $\left\{E_{n}\right\}_{n=1}^{\infty}$ e

$$
\begin{gathered}
\left\|T\left(e_{n}, 0\right)\right\|_{\beta}-\epsilon_{n}>c . \\
\left\|T\left(0, e_{n}\right)\right\|_{\beta}-\epsilon_{n}>c .
\end{gathered}
$$

Do fato que $\lim _{n \rightarrow \infty}\left\|w \xi_{B_{n}}\right\|_{2}=0$, concluímos que existe uma sequência crescente $k=\left(k_{n}\right)_{n}$ de inteiros positivos tal que $\left\|w \xi_{B_{k_{n}}}\right\|_{2} \leq \epsilon_{n}$ para todo $n \in \mathbb{N}$. Seja $T_{k}$ o operador associado à sequência $k$, como na observação $(3.2)$, se $T_{k}^{n}$ denota o operador $T_{k}$ restrito ao subespaço $E_{n}$, então

$$
W_{k_{n}} T_{k}^{n}: E_{n} \rightarrow Z_{2}(\beta)
$$

satisfaz $\left\|W_{k_{n}} T_{k}^{n}\right\| \leq\left\|W_{k_{n}}\right\|\left\|T_{k}^{n}\right\| \leq \epsilon_{n}$. Pelo Lema (1.53), o operador $W=\sum_{n} W_{k_{n}} T_{k}^{n}$ é contínuo e $\|W\| \leq \epsilon$. Escrevamos $S=T \circ T_{k}-W$, então

- $\|S\| \leq\left\|T \circ T_{k}\right\|+\epsilon \leq\|T\|+\epsilon<1$

- $\left\|S\left(e_{n}, 0\right)\right\|_{\beta}=\left\|T\left(e_{k_{n}}, 0\right)\right\|_{\beta}>c$

- $\|\left(S\left(0, e_{n}\right)\left\|_{\beta} \geq\right\| T\left(0, e_{k_{n}}\right) \|_{\beta}-\epsilon_{k_{n}}>c\right.$

e observemos que neste caso

$$
\begin{aligned}
S\left(0, e_{n}\right) & =\left(w \xi_{B_{n}}, y \xi_{B_{n}}\right)-\left(w \xi_{B_{n}}, \Omega_{\beta}\left(w \xi_{B_{n}}\right)\right) \\
& =\left(0, \widetilde{y} \xi_{B_{n}}\right) .
\end{aligned}
$$

Assim, $S$ é $c$-admissível com as propriedades da afirmação 1 e tal que $w=0$. Vamos conser- 
var a notação de $T$ para o operador achado.

Afirmação 4. Agora mostraremos que o operador construido na afirmação anterior satisfaz $\inf _{n \in \mathbb{N}}\left\|u \xi_{B_{n}}\right\|_{\infty} \neq 0$.

Supondo o contrário, dado $K>0$ existe uma sequência crescente $\left(k_{n}\right)_{n=1}^{\infty}$ de inteiros tais que $\left\|u \xi_{B_{k_{n}}}\right\|_{\infty} \leq e^{-K}$. Mais uma vez, pelo argumento da subsequência dado pela observação (3.2), podemos assumir que existe um operador admissível $T: Z_{2}(\alpha) \rightarrow Z_{2}(\beta), T=T_{K}$ $(\operatorname{com} w=0)$ e tal que $\|u\|_{\infty} \leq e^{-K}$.

Com isto, sejam $m_{1}<M_{1}$ e $m_{2}<M_{2}$ pares de inteiros e definamos $\sigma_{r}=1 / 2 \log m_{r}$ e $\tau_{r}=1 / 2 \log M_{r}$ para $r=1,2$. Seja $M$ qualquer inteiro maior do que $\max \left\{M_{1}, M_{2}\right\}$. Suponha $A \subseteq\{1, \ldots, M\}$ com $|A|=m_{1}$. Então $\left\|\sigma_{1}^{\alpha} \xi_{A}-\Omega_{\alpha}\left(\xi_{A}\right)\right\|_{2}=0$, pois

$$
\Omega_{\alpha}\left(\xi_{A}\right)(k)=\xi_{A}(k)\left(\log \frac{\left\|\xi_{A}\right\|_{2}}{\left|\xi_{A}(k)\right|}\right)^{\alpha}=\left(\log m_{1}^{1 / 2}\right)^{\alpha}=\sigma_{1}^{\alpha}
$$

$\operatorname{Logo}\left\|\left(\xi_{A}, \sigma_{1}^{\alpha} \xi_{A}\right)\right\|_{\alpha}=\left\|\xi_{A}\right\|=m_{1}^{1 / 2}$. Observemos que

$$
\begin{aligned}
T\left(m_{1}^{-1 / 2}\left(\xi_{A}, \xi_{1}^{\alpha} \xi_{A}\right)\right) & =\sum_{k \in A} m_{1}^{-1 / 2}\left(T\left(e_{k}, 0\right)+\sigma_{1}^{\alpha} T\left(0, e_{k}\right)\right) \\
& =\sum_{k \in A} m_{1}^{-1 / 2}\left(\left(u \xi_{B_{k}}, v \xi_{B_{k}}\right)+\sigma_{1}^{\alpha}\left(0, y \xi_{B_{k}}\right)\right) \\
& =\sum_{k \in A} m_{1}^{-1 / 2}\left(u \xi_{B_{k}}, v \xi_{B_{k}}+\sigma_{1}^{\alpha} y \xi_{B_{k}}\right)
\end{aligned}
$$

como $\|T\| \leq 1$, então

$$
\left\|\sum_{k \in A} m_{1}^{-1 / 2}\left(u \xi_{B_{k}}, v \xi_{B_{k}}+\sigma_{1}^{\alpha} y \xi_{B_{k}}\right)\right\|_{\beta} \leq 1
$$

e em consequência

$$
\begin{gathered}
\left\|\sum_{k \in A} m_{1}^{-1 / 2}\left(v \xi_{B_{k}}+\sigma_{1}^{\alpha} y \xi_{B_{k}}\right)-\Omega_{\beta}\left(\sum_{k \in A} m_{1}^{-1 / 2} u \xi_{B_{k}}\right)\right\|_{2} \leq 1 \\
\left\|\sum_{k \in A} m_{1}^{-1 / 2} u \xi_{B_{k}}\right\|_{2} \leq 1 .
\end{gathered}
$$

Usamos (3.4) e a anterior desigualdade para obter

$$
\left\|\Omega_{\beta}\left(\sum_{k \in A} m_{1}^{-1 / 2} u \xi_{B_{k}}\right)-\Omega_{\beta}^{\prime}\left(\sum_{k \in A} m_{1}^{-1 / 2} u \xi_{B_{k}}\right)\right\|_{2} \leq|b|
$$


e logo por (3.12)

$$
\left\|m_{1}^{-1 / 2} \sigma_{1}^{\alpha} \sum_{k \in A} y \xi_{B_{k}}+m_{1}^{-1 / 2} \sum_{k \in A} v \xi_{B_{k}}-\Omega_{\beta}^{\prime}\left(\sum_{k \in A} m_{1}^{-1 / 2} u \xi_{B_{k}}\right)\right\|_{2} \leq 1+|b|
$$

Para simplificar notação escrevamos $\rho_{j}=-\log |u(j)|$ se $|u(j)| \neq 0$ e $\rho_{j}=K$ se $|u(j)|=0$. Desta forma, $\rho_{j} \geq K$ pois $|u(j)| \leq e^{-K}$. Agora, desenvolvendo a equação anterior

$$
\begin{aligned}
\Omega_{\beta}^{\prime}\left(\sum_{k \in A} m_{1}^{-1 / 2} u \xi_{B_{k}}\right) & =m_{1}^{-1 / 2} u(j)\left(\log \left|m_{1}^{-1 / 2} \sum_{k \in A} u \xi_{B_{k}}(j)\right|^{-1}\right)^{b} \\
& =m_{1}^{-1 / 2} u(j)\left(\log m_{1}^{1 / 2}+\log |u(j)|^{-1}\right)^{b} \\
& =m_{1}^{-1 / 2} u(j)\left(\sigma_{1}+\rho_{j}\right)^{b} .
\end{aligned}
$$

Então

$$
\frac{1}{m_{1}} \sum_{k \in A} \sum_{j \in B_{k}}\left|\sigma_{1}^{a} y(j)+v(j)-\left(\sigma_{1}+\rho_{j}\right)^{b} u(j)\right|^{2} \leq(1+|b|)^{2} .
$$

Logo, para cada $i \in\left\{1, \ldots, m_{1}+1\right\}$

$$
\sum_{k=1, k \neq i}^{m_{1}+1} \sum_{j \in B_{k}}\left|\sigma_{1}^{a} y(j)+v(j)-\left(\sigma_{1}+\rho_{j}\right)^{b} u(j)\right|^{2} \leq m_{1}(1+|b|)^{2}
$$

e somando as $m_{1}+1$ desigualdades obtidas

$$
m_{1} \sum_{k=1}^{m_{1}+1} \sum_{j \in B_{k}}\left|\sigma_{1}^{a} y(j)+v(j)-\left(\sigma_{1}+\rho_{j}\right)^{b} u(j)\right|^{2} \leq\left(m_{1}+1\right) m_{1}(1+|b|)^{2} .
$$

Portanto

$$
\frac{1}{m_{1}+1} \sum_{k=1}^{m_{1}+1} \sum_{j \in B_{k}}\left|\sigma_{1}^{a} y(j)+v(j)-\left(\sigma_{1}+\rho_{j}\right)^{b} u(j)\right|^{2} \leq(1+|b|)^{2}
$$

e em consequência,

$$
\frac{1}{M} \sum_{k=1}^{M} \sum_{j \in B_{k}}\left|\sigma_{1}^{a} y(j)+v(j)-\left(\sigma_{1}+\rho_{j}\right)^{b} u(j)\right|^{2} \leq(1+|b|)^{2} .
$$

De maneira análoga

$$
\frac{1}{M} \sum_{k=1}^{M} \sum_{j \in B_{k}}\left|\tau_{1}^{a} y(j)+v(j)-\left(\tau_{1}+\rho_{j}\right)^{b} u(j)\right|^{2} \leq(1+|b|)^{2}
$$


Usando a estimativa (3.3) do Lema (3.3), temos

$$
\begin{gathered}
\left|\left(s+\rho_{j}\right)^{b}-\rho_{j}^{b}-b s \rho_{j}^{b-1}\right| \leq \frac{|b| s^{2}}{2 K} \\
\left|\left(\tau_{1}+\rho_{j}\right)^{b}-\left(\sigma_{1}+\rho_{j}\right)^{b}-b\left(\tau_{1}-\sigma_{1}\right) \rho_{j}^{b-1}\right| \leq \frac{|b| \tau_{1}^{2}}{K}
\end{gathered}
$$

Definamos

$$
\begin{aligned}
\kappa & =\sum_{k=1}^{M} \sum_{j \in B_{k}} \sigma_{1}^{a} y(j)+v(j)-\left(\sigma_{1}+\rho_{j}\right)^{b} u(j) \\
\lambda & =\sum_{k=1}^{M} \sum_{j \in B_{k}} \tau_{1}^{a} y(j)+v(j)-\left(\tau_{1}+\rho_{j}\right)^{b} u(j) \\
\mu & =\sum_{k=1}^{M} \sum_{j \in B_{k}}\left(\tau_{1}+\rho_{j}\right)^{b}-\left(\sigma_{1}+\rho_{j}\right)^{b}-b\left(\tau_{1}-\sigma_{1}\right) \rho_{j}^{b-1} u(j)
\end{aligned}
$$

Assim, levando em conta que $\left\|u \xi_{B_{k}}\right\|_{\infty} \leq 1$

$$
\begin{aligned}
\|\lambda-\kappa+\mu\|_{2} & \leq\|\lambda\|_{2}+\|\kappa\|_{2}+\|\mu\|_{2} \\
& \leq 2 \sqrt{M}(1+|b|)+M|b| \tau_{1}^{2} / K
\end{aligned}
$$

Portanto,

$$
\begin{aligned}
& \frac{1}{M} \sum_{k=1}^{M} \sum_{j \in B_{k}}\left|\tau_{1}^{a} y(j)-\sigma_{1}^{a} y(j)-b \rho_{j}^{i \beta} u(j)\left(\tau_{1}-\sigma_{1}\right)\right|^{2} \\
\leq & \left(\frac{2 \sqrt{M}}{M^{2}}(1+|b|)+\frac{M K^{-1}}{M^{2}}|b| \tau_{1}^{2}\right)^{2} \\
\leq & \left(2(1+|b|)+K^{-1}|b| \tau_{1}^{2}\right)^{2}
\end{aligned}
$$

de onde

$$
\frac{1}{M} \sum_{k=1}^{M} \sum_{j \in B_{k}}\left|\frac{\tau_{1}^{a}-\sigma_{1}^{a}}{\tau_{1}-\sigma_{1}} y(j)-b \rho_{j}^{i \beta} u(j)\right|^{2} \leq \frac{\left(2(1+|b|)+K^{-1}|b| \tau_{1}^{2}\right)^{2}}{\left(\tau_{1}-\sigma_{1}\right)^{2}}
$$

Um cálculo similar ao feito até agora, mostra uma equação análoga à anterior para $\sigma_{2}$ e $\tau_{2}$.

$$
\frac{1}{M} \sum_{k=1}^{M} \sum_{j \in B_{k}}\left|\frac{\tau_{2}^{a}-\sigma_{2}^{a}}{\tau_{2}-\sigma_{2}} y(j)-b \rho_{j}^{i \beta} u(j)\right|^{2} \leq \frac{\left(2(1+|b|)+K^{-1}|b| \tau_{2}^{2}\right)^{2}}{\left(\tau_{2}-\sigma_{2}\right)^{2}}
$$

Lembremos que $\left\|T\left(0, e_{n}\right)\right\|_{\beta} \geq c \log \mathrm{O}$

$$
\frac{1}{M} \sum_{k=1}^{M} \sum_{j \in B_{k}}|y(j)|^{2} \geq c^{2}
$$


Pela desigualdade triangular e as equações (3.13), (3.14)

$$
\frac{1}{M} \sum_{k=1}^{M} \sum_{j \in B_{k}}\left|\frac{\tau_{1}^{a}-\sigma_{1}^{a}}{\tau_{1}-\sigma_{1}}-\frac{\tau_{2}^{a}-\sigma_{2}^{a}}{\tau_{2}-\sigma_{2}}\right|^{2}|y(j)|^{2} \leq L_{1}+L_{2}
$$

onde $L_{r}=\frac{\left(2(1+|b|)+K^{-1}|b| \tau_{r}^{2}\right)^{2}}{\left(\tau_{r}-\sigma_{r}\right)^{2}}$ para $r=1,2$. Portanto

$$
\left|\frac{\tau_{1}^{a}-\sigma_{1}^{a}}{\tau_{1}-\sigma_{1}}-\frac{\tau_{2}^{a}-\sigma_{2}^{a}}{\tau_{2}-\sigma_{2}}\right| \leq N_{1}+N_{2}
$$

onde

$$
N_{r}=\frac{1}{c}\left(K^{-1}|b| \tau_{r}^{2}+2+2|b|\right)
$$

Seja $k>1$ um inteiro, é claro que $k^{\alpha} \neq 1$. Vamos mostrar que

$$
\lim _{n \rightarrow \infty} \frac{k^{a} \varsigma_{n}^{a}-\varsigma_{n}^{a}}{k \varsigma_{n}-\varsigma_{n}}
$$

existe, onde $\varsigma_{n}=(1 / 2) \log n$.

Sejam $n, m \in \mathbb{N}$ arbitrários, escrevamos

$$
\begin{array}{lll}
m_{1}=n & \text { e } & M_{1}=n^{k} \\
m_{2}=n & \text { e } & M_{2}=n^{k}
\end{array}
$$

Com isto, $\sigma_{1}=(1 / 2) \log m_{1}=\varsigma_{n}$ e $\tau_{1}=(1 / 2) \log M_{1}=k \varsigma_{n}$, de forma análoga, $\sigma_{2}=\varsigma_{m}$ e $\tau_{2}=k \varsigma_{m}$. Como $K$ é arbitrário na fórmula (3.16), então

$$
\left|\frac{k^{a} \varsigma_{n}^{a}-\varsigma_{n}^{a}}{k \varsigma_{n}-\varsigma_{n}}-\frac{k^{a} \varsigma_{m}^{a}-\varsigma_{m}^{a}}{k \varsigma_{m}-\varsigma_{m}}\right| \leq \frac{c^{\prime}}{\sigma_{1}}+\frac{c^{\prime}}{\sigma_{2}}
$$

para $c^{\prime}>0$ uma constante. Isto implica que o limite em (3.17) existe, pois a sequência é de Cauchy. Portanto, $\lim _{n \rightarrow \infty} \varsigma_{n}^{i \alpha}\left(\frac{k^{\alpha}-1}{k-1}\right)$ existe. Em outras palavras

$$
\lim _{n \rightarrow \infty}(\log n)^{i \alpha}
$$

existe. Vamos provar que isto é impossivel se $\alpha \neq 0$. Com efeito, pelo seguinte lema, para cada $t>0$ podemos obter uma subsequência $\left(\log n_{k}\right)_{n_{k}}^{i \alpha}$ que converge para $e^{i \alpha t}$.

Lema 3.7. Seja $\left(t_{n}\right)_{n}$ uma sequência de números reais tal que $\lim _{n \rightarrow \infty} t_{n}=+\infty$ e $\lim _{n \rightarrow \infty} t_{n+1}-$ $t_{n}=0$. Então para cada $t \in \mathbb{R}^{+}$e cada $\epsilon>0$, existe $n_{0} \in \mathbb{N}$ tal que $\left|(t+2 s \pi)-t_{n_{0}}\right|<\epsilon$ para algum inteiro $s>0$.

Antes de demonstrar o lema, vejamos como usá-lo. Temos que $(\log n)^{i \alpha}=e^{i \alpha(\log n)}$ e que a sequência $t_{n}=\alpha \log n$ satisfaz as hipóteses do lema, logo para cada $t>0$ existem sequências 
crescentes $\left(s_{k}\right)_{k}$ e $\left(n_{k}\right)_{k}$ de inteiros positivos tais que

$$
\lim _{k \rightarrow \infty} t_{n_{k}}-2 s_{k} \pi=t
$$

Da continuidade da função $f(\theta)=e^{i \theta}$, obtemos $\lim _{k \rightarrow \infty} e^{i t_{n_{k}}}=\lim _{k \rightarrow \infty} e^{i\left(t_{n_{k}}-2 s_{k} \pi\right)}=e^{i \alpha t}$. Assim, o limite em (3.17) não existe.

Portanto, temos provado que existe um operador admissível $T$ com as propriedades da afirmação 1 , tal que $w=0$ e inf $\left\|u \xi_{B_{n}}\right\|_{\infty}>0$.

Demonstração. (Lema (3.7)) Seja $M \in \mathbb{N}$ tal que $\left|t_{n+1}-t_{n}\right|<\epsilon$ para todo $n \geq M$. Agora como $\lim _{n \rightarrow \infty} t_{n}=+\infty$, seja $s>0$ um inteiro tal que se $t_{n+1} \geq t+2 s \pi$ implica $n+1 \geq M$ e $t_{1}<t+2 s \pi$. Logo tomemos $n_{0}=\max \left\{n \in \mathbb{N} ; \quad t_{n}<t+2 s \pi\right\}$. Assim $\left|t_{n_{0}+1}-t_{n_{0}}\right|<\epsilon$ pois $n_{0}+1 \geq M \mathrm{e}$

$$
\left|t+2 s \pi-t_{n_{0}}\right|<\left|t_{n_{0}+1}-t_{n_{0}}\right|<\epsilon
$$

Afirmação 5. Agora o objetivo será mostrar que existe um operador linear contínuo $S: Z_{2}(\alpha) \rightarrow Z_{2}(\beta)$ com $\|S\|<1$ tal que $S\left(e_{n}, 0\right)=\left(\lambda e_{n}, \mu e_{n}\right)$ e $S\left(0, e_{n}\right)=\left(0, \nu e_{n}\right)$ para constantes $\lambda, \mu$ e $\nu \operatorname{com} \lambda \neq 0$.

Como as sequências $u, v$ e $y$ são limitadas, podemos passar a uma subsequência onde cada uma converge. Vamos perturbar $T$ no sentido do Lema (1.53). Usando o argumento de subsequência(Observação (3.2)), podemos assumir para cada $n \in \mathbb{N}:\left|u_{n}-\lambda\right|<\epsilon_{n},\left|v_{n}-\mu\right|<\epsilon_{n}$ e $\left|y_{n}-\nu\right|<\epsilon_{n}$ para $\epsilon_{n}$ tais que $\sum \epsilon_{n}=\epsilon$ seja suficientemente pequeno(tal que $\|T\|+3 \epsilon<1$ ). Notemos que $\lambda \neq 0$ pois inf $\left\|u \xi_{B_{n}}\right\|_{\infty}>0$. Definamos $W_{n}: E_{n} \rightarrow Z_{2}(\beta)$ primeiro na base de $E_{n}$ :

$$
\begin{aligned}
& T_{n}\left(e_{n}, 0\right)=\left(\left(u_{n}-\lambda\right) e_{n},\left(v_{n}-\mu\right) e_{n}\right) \\
& T_{n}\left(0, e_{n}\right)=\left(0,\left(y_{n}-\nu\right) e_{n}\right)
\end{aligned}
$$

e estenda por linearidade. Assim

$$
\begin{aligned}
\left\|W_{n}\left(c_{n} e_{n}, d_{n} e_{n}\right)\right\|_{\beta} & =\left|c_{n}\right|\left(\left|u_{n}-\lambda\right|+\left|v_{n}-\mu\right|\right)+\left|d_{n}\right|\left(\left|y_{n}-\nu\right|\right) \\
& \leq 3 \epsilon_{n}\left\|\left(c_{n} e_{n}, d_{n} e_{n}\right)\right\|_{\alpha} .
\end{aligned}
$$

Logo é consequência do Lema (1.53) que $W=\sum_{n} W_{n}$ é contínuo e $\|T-W\| \leq\|T\|+3 \epsilon<1$. E neste caso, $S=T-W$ satisfaz

$$
\begin{aligned}
& S\left(e_{n}, 0\right)=\left(\lambda e_{n}, \mu e_{n}\right) \\
& S\left(0, e_{n}\right)=\left(0, \nu e_{n}\right)
\end{aligned}
$$


para todo $n \in \mathbb{N}$. O que completa a prova da afirmação.

Afirmação 6. Vamos concluir que se existe um operador linear contínuo $S: Z_{2}(\alpha) \rightarrow$ $Z_{2}(\beta)$ com as propriedades da afirmação 5 , então devemos ter $\alpha=\beta$.

Para cada $N \in \mathbb{N}$, definamos o conjunto $A=\{1, \ldots, N\}$. Anteriormente observamos que

$$
\left\|\left(N^{-1 / 2} \xi_{A}, \sigma_{N}^{a} N^{-1 / 2} \xi_{A}\right)\right\|_{\alpha}=1
$$

onde $\sigma_{N}=\frac{1}{2} \log N$. Portanto

$$
\left\|S\left(N^{-1 / 2} \xi_{A}, \sigma_{N}^{a} N^{-1 / 2} \xi_{A}\right)\right\|_{\beta} \leq 1
$$

Desenvolvendo a equação anterior, obtemos

$$
N^{-1 / 2}\left\|\left(\sigma_{N}^{a} \nu+\mu\right) \xi_{A}-\lambda(\log \sqrt{N})^{b} \xi_{A}\right\|_{2}<1
$$

isto é

$$
\left|\sigma_{N}^{a} \nu+\mu-\lambda \sigma_{N}^{b}\right| \leq 1
$$

Portanto, $\sigma_{N}^{a} \nu-\lambda \sigma_{N}^{b}$ é limitado. Fatorizando, observamos que

$$
\sigma_{N} e^{i \alpha \log \sigma_{N}}\left(\nu-\lambda e^{i(\beta-\alpha) \log \sigma_{N}}\right)
$$

é limitado.

Suponhamos que $\beta \neq \alpha$ (sem perda de generalidade $\beta>\alpha)$, escrevendo $t_{N}=(\beta-\alpha) \log \sigma_{N}$ a expressão anterior implica que a sequência

$$
\sigma_{N} e^{i \alpha \frac{t_{N}}{\beta-\alpha}}\left(\nu-\lambda e^{i t_{N}}\right)
$$

é limitada. Notemos que o fator $\sigma_{N}$ não é limitado.

Observemos que $\left(t_{N}\right)_{N}$ satisfaz as condições do Lema (3.7) e portanto existem sequências crescentes $\left(s_{k}\right)$ e $\left(N_{k}\right)$ de inteiros positivos, tais que

$$
\lim _{k \rightarrow \infty} t_{N_{k}}-2 s_{k} \pi=2 \pi
$$

Logo, pela continuidade de $e^{i \theta}$, temos $1=e^{2 \pi i}=\lim _{k \rightarrow \infty} e^{\left(t_{N_{k}}-2 s_{k} \pi\right) i}=\lim _{k \rightarrow \infty} e^{t_{N_{k}} i}$. Assim

$$
\lim _{k \rightarrow \infty} \nu-\lambda e^{i t_{N_{k}}}=\nu-\lambda
$$

Como a expressão (3.18) é limitada, então devemos ter $\nu=\lambda$. 
Agora, aplicando um argumento análogo ao anterior, existe uma subsequência $\left(t_{N_{j}}\right)_{j}$ tal que

$$
\lim _{j \rightarrow \infty} t_{N_{j}}-2 s_{j} \pi=\pi
$$

e $\operatorname{assim}-1=\lim _{j \rightarrow \infty} e^{i t_{N_{j}}}$. Portanto

$$
\lim _{j \rightarrow \infty} \nu-\lambda e^{i t_{N_{j}}}=\nu+\lambda
$$

e $\nu+\lambda \neq 0$. Isto contradiz o fato que a sequência em (3.18) é limitada. Logo devemos ter $\alpha=\beta$.

A prova do Teorema (3.6) está completa.

Corolario 3.8. O espaço $Z_{2}(\alpha)$ não é isomorfo a seu conjugado complexo se $\alpha \neq 0$. 


\section{Referências Bibliográficas}

[Bou86] J. Bourgain. Real isomorphic complex banach spaces need not be complex isomorphic. Proc. Amer. Math. Soc, 96:221-226, 1986. 1

[CG97] J. M. Castillo e M. Gonzalez. Three-space problems in Banach space theory. Lect. Notes in Math. Springer Verlag, vol. 1667 edição, 1997. 30

[Fea01] Marián Fabian e et al. Functional Analysis and Infinite-dimensional Geometry. Springer Verlag, New York, 2001. 7, 18

[Jam50] R. C. James. Bases and reflexivity of banach spaces. Ann. of Math., 52:518-527, 1950. 18

[Kal78] N.J Kalton. The three space problem for locally bounded f-spaces. Compositio Mathematica, 37 No. 3:243-276, 1978. 1

[Kal95] N.J. Kalton. An elementary example of a banach space not isomorphic to its complex conjugate. Canad. Math. Bull, 38:218-222, 1995. iii, v, 1, 2

[KP79] N.J Kalton e N.T Peck. Twisted sums of sequence spaces and the three space problem. Trans. Amer. Math. Soc., 255:1-30, 1979. 1

[KPR84] N. J. Kalton, N. T. Peck, e James W. Roberts. An F-space sampler. Cambridge: Cambridge UP, 1984. 14

[LT77] Joram Lindenstrauss e Lior Tzafriri. Clasical Banach Spaces I, Sequence spaces. Springer Verlag, Berlin, 1977. 19

[Meg98] R. Megginson. An Introduction to Banach Space Theory. Springer Verlag, New York, 1998. 5, 6

[MU32] S. Mazur e S. Ulam. Sur les transformations isométriques d'espaces vectoriels normés. C. R. Acad. Sci. Paris, 194:946-948, 1932. 1

[Rib79] M. Ribe. Examples for the non-locally convex three space problem. Proc. Amer. Math. Soc., 73 No.3:351-355, 1979. 1, 25, 30

[Rud73] Walter Rudin. Functional Analysis. McGraw-Hill, New York, 1973. 8 